\title{
Rational functions with maximal radius of absolute monotonicity
}

\author{
Lajos Lóczi and David I. Ketcheson
}

\begin{abstract}
We study the radius of absolute monotonicity $R$ of rational functions with numerator and denominator of degree $s$ that approximate the exponential function to order $p$. Such functions arise in the application of implicit $s$-stage, order $p$ Runge-Kutta methods for initial value problems, and the radius of absolute monotonicity governs the numerical preservation of properties like positivity and maximum-norm contractivity. We construct a function with $p=2$ and $R>2 s$, disproving a conjecture of van de Griend and Kraaijevanger. We determine the maximum attainable radius for functions in several one-parameter families of rational functions. Moreover, we prove earlier conjectured optimal radii in some families with two or three parameters via uniqueness arguments for systems of polynomial inequalities. Our results also prove the optimality of some strong stability preserving implicit and singly diagonally implicit Runge-Kutta methods. Whereas previous results in this area were primarily numerical, we give all constants as exact algebraic numbers.
\end{abstract}

\section{Introduction and aims}

A real function $\psi$ is said to be absolutely monotonic at a point $x \in \mathbb{R}$, if the $k$ th derivative of $\psi$ at $x, \psi^{(k)}(x)$, exists and is non-negative for each integer $k \geqslant 0$. The radius of absolute monotonicity $R(\psi) \in[0,+\infty]$ is defined as

$$
R(\psi)=\sup (\{r \in[0,+\infty): \psi \text { is absolutely monotonic at each point of }[-r, 0]\} \cup\{0\}) .
$$

The radius of absolute monotonicity of polynomials and rational functions plays an important role in the analysis of positivity, monotonicity, and contractivity properties of numerical methods for initial value problems and is often referred to as the threshold factor in this context $[\mathbf{1}, \mathbf{5}, \mathbf{7}, \mathbf{1 0}, \mathbf{1 2}]$. Specifically, the maximal positive or contractive step-size is given by $R(\psi) h_{0}$, where $\psi$ is the stability function of the numerical method and $h_{0}$ is the maximum step-size under which the corresponding property holds for the explicit Euler method.

It is therefore natural to consider the problem of finding a function $\psi$ that achieves the maximal radius of absolute monotonicity within a given class. In this work, we study absolute monotonicity of rational functions that correspond to the stability functions of certain implicit or singly diagonally implicit Runge-Kutta methods.

A Runge-Kutta (RK) method of $s \in \mathbb{N}^{+}$stages is defined by its coefficients: an $s \times s$ matrix $A$ and an $s \times 1$ vector $b[2]$. The stability function of the method is

$$
\psi(z)=\psi^{A, b}(z):=\frac{\operatorname{det}\left(I-z A+z \mathbb{1} b^{\top}\right)}{\operatorname{det}(I-z A)} \quad(z \in \mathbb{C}),
$$

where $\mathbb{1}$ is a column vector of length $s$ with all unit entries and $I$ is the $s \times s$ identity matrix [6, $\S$ IV.3]. The order of a method, denoted by $p \in \mathbb{N}^{+}$, indicates how accurately the computed

Received 22 March 2013; revised 12 September 2013.

2010 Mathematics Subject Classification 65L06 (primary), 68W30, 41A20, 49K30 (secondary).

This publication is based on work supported by Award No. FIC/2010/05 - 2000000231, made by King Abdullah University of Science and Technology (KAUST). 
solution approximates the exact solution in an asymptotic sense. The stability function of an RK method of order $p$ must approximate the exponential function to at least order $p$ near the origin.

In an implicit Runge-Kutta method (IRK), all entries of $A$ may be non-zero. An important subclass of the IRK methods are the singly diagonally implicit RK (SDIRK) methods, with $A$ lower-triangular with identical diagonal entries. For explicit $R K$ methods, $A$ is strictly lowertriangular.

We now define the classes of rational functions to be studied. For $m, n \in \mathbb{N}$, let $\Pi_{m}$ denote the set of real polynomials of degree at most $m$,

$$
\Pi_{m}=\left\{\sum_{j=0}^{m} \beta_{j} z^{j}: \beta_{j} \in \mathbb{R}, j=0,1, \ldots, m\right\},
$$

and let $\Pi_{m / n, p}$ denote the set of all real $(m, n)$-rational functions approximating the exponential to order $p$ near the origin,

$$
\Pi_{m / n, p}=\left\{\psi: \psi=\frac{P}{Q}, P \in \Pi_{m}, 0 \not \equiv Q \in \Pi_{n}, \psi(z)-\exp (z)=\mathcal{O}\left(z^{p+1}\right) \text { as } z \rightarrow 0\right\} .
$$

Let $\widehat{\Pi}_{m / n, p}$ denote the elements of $\Pi_{m / n, p}$ whose denominator has (at most) a single, non-zero real root

$$
\widehat{\Pi}_{m / n, p}=\left\{\psi \in \Pi_{m / n, p}: \psi(z)=\frac{P(z)}{(1-a z)^{n}}, P \in \Pi_{m}, a \in \mathbb{R}\right\} .
$$

REMARK 1.1. Obviously, for every $0 \leqslant m \leqslant \widetilde{m}, 0 \leqslant n \leqslant \widetilde{n}$ and $1 \leqslant p \leqslant \widetilde{p}$, we have $\widehat{\Pi}_{m / n, p} \subset \Pi_{m / n, p}, \Pi_{m / n, p} \subset \Pi_{\widetilde{m} / \widetilde{n}, p}$ and $\Pi_{m / n, p} \supset \Pi_{m / n, \widetilde{p}}$.

If $A$ and $b$ correspond to an IRK method of $s$ stages and order $p$, then

$$
\psi^{A, b} \in \Pi_{s / s, p},
$$

while for SDIRK methods,

$$
\psi^{A, b} \in \widehat{\Pi}_{s / s, p} .
$$

For explicit RK methods we have $\psi^{A, b} \in \Pi_{s}$.

A thorough study of polynomial approximations to the exponential with maximal radius of absolute monotonicity can be found in $[\mathbf{7}, \mathbf{1 0}]$. We are interested in determining the maximal radius of absolute monotonicity that can be achieved among the stability functions of IRK or SDIRK methods of a given order $p$. Therefore, for non-empty sets $\Pi_{m / n, p}$ and $\widehat{\Pi}_{m / n, p}$, let us define the quantities

$$
\begin{aligned}
& R_{m / n, p}:=\sup \left\{R(\psi): \psi \in \Pi_{m / n, p}\right\}, \\
& \widehat{R}_{m / n, p}:=\sup \left\{R(\psi): \psi \in \widehat{\Pi}_{m / n, p}\right\} .
\end{aligned}
$$

We will focus on the cases for which $m=n=s$.

REMARK 1.2. Generically, the class $\Pi_{s / s, p}$ can be written as a family in $2 s-p$ parameters, while the class $\widehat{\Pi}_{s / s, p}$ can be written as a family in $s+1-p$ parameters. In the cases where there are zero free parameters, these sets contain a finite number of members. Throughout the paper, 'finitely many' in this context is understood in the sense of functions, that is, a normalized representation is chosen: if $\psi=P / Q$ with $\psi(0)=1$, then we can assume $P(0)=Q(0)=1$. 
The seminal work on this topic is [5], in which an algorithm is presented for computing the radius of absolute monotonicity of a rational function, and many properties of the radius of absolute monotonicity are proved. The determination of $R(\psi)$ is not trivial even for a single rational function $\psi$, so the difficulty of obtaining $R_{m / n, p}$ or $\widehat{R}_{m / n, p}$ for a particular $(m, n, p)$ triple ranges from fairly challenging to currently impossible. Nevertheless, some patterns in numerically computed values have led to important conjectures.

In order to describe these conjectures, we recall the concept of radius of absolute monotonicity of a Runge-Kutta method [11], denoted by $R(A, b)$. The quantity $R(A, b)$ is also referred to as Kraaijevanger's coefficient [3], or the SSP coefficient [4]. For an RK method with coefficients $A, b$, define $K \in \mathbb{R}^{(s+1) \times(s+1)}$ by

$$
K=K(A, b):=\left(\begin{array}{cc}
A & 0 \\
b^{\top} & 0
\end{array}\right) .
$$

Now let $\mathbb{1}$ denote the vector $(1,1, \ldots, 1)^{\top} \in \mathbb{R}^{s+1}$. Then the radius of absolute monotonicity of the RK method is

$$
R(A, b):=\sup \left\{r \in \mathbb{R}: \forall \varrho \in[0, r] \exists(I+\varrho K)^{-1}, \varrho K(I+\varrho K)^{-1} \geqslant 0 \text { and } \varrho K(I+\varrho K)^{-1} \mathbb{1} \leqslant \mathbb{1}\right\},
$$

where vector and matrix inequalities are understood componentwise. Notice that $R(A, b) \geqslant 0$. Absolute monotonicity of a Runge-Kutta method implies absolute monotonicity of its stability function [11]; thus we have

$$
R(A, b) \leqslant R\left(\psi^{A, b}\right)
$$

The coefficient $R(A, b)$ plays the same role in numerical preservation of positivity and contractivity for non-linear problems that the coefficient $R\left(\psi^{A, b}\right)$ plays for linear problems [4].

The following conjectures served as motivation for our work.

Conjecture $1.3\left[\mathbf{5}\right.$, p. 421]. For $m, n \in \mathbb{N}^{+}, R_{m / n, 2}=m+\sqrt{m n}$.

In [5], this conjecture was proved for all $m \geqslant 1$ with $n=1$ or 2 . In the special case $m=n=s \in \mathbb{N}^{+}$, the conjecture claims

$$
R_{s / s, 2}=2 s .
$$

For $A, b$ corresponding to an RK method with $s$ stages and order $p$, we have $R\left(\psi^{A, b}\right) \leqslant R_{s / s, p}$. Moreover, $R_{s / s, p}$ is a non-increasing function of $p$ for fixed $s$; therefore (1.4) and (1.5) together would imply that $R(A, b) \leqslant 2 s$ for all Runge-Kutta methods that are more than first order accurate. Indeed, evidence in the literature reinforces belief in the bound (1.5); we have the following conjectures based on numerical searches.

Conjecture 1.4 [3, Conjecture 3.1]. Let $A, b$ denote the coefficients of an SDIRK method of order $p \geqslant 2$ with $s \geqslant 1$ stages. Then $R(A, b) \leqslant 2 s$.

Conjecture 1.5 [9]. Let $A, b$ denote the coefficients of an implicit RK method of order $p \geqslant 2$ with $s \geqslant 1$ stages. Then $R(A, b) \leqslant 2 s$.

It is thus very surprising that, as we will see, Conjecture 1.3, and thus equality (1.5), does not hold for $m=n=3$. An immediate consequence is that Conjecture 1.5 cannot be proved by analyzing $R_{s / s, 2}$. The following is another conjecture that arises naturally.

Conjecture 1.6. For each $s \geqslant 1, \widehat{R}_{s / s, 2}=2 s$. 
We have presented these conjectures in the order they were formulated. By also taking into account that:

- $\widehat{R}_{s / s, p} \leqslant \widehat{R}_{s / s, 2} \leqslant R_{s / s, 2}(p \geqslant 2)$;

- $R\left(\psi^{A, b}\right) \leqslant \widehat{R}_{s / s, p}$ for $A, b$ corresponding to an SDIRK method;

- there exists an SDIRK method (consisting of the repetitions of the implicit midpoint method) with $R(A, b)=2 s[3$, formula (3.1)] whose stability function

$$
\psi^{A, b}(z)=\frac{(1+z /(2 s))^{s}}{(1-z /(2 s))^{s}}, \quad \psi^{A, b} \in \widehat{\Pi}_{s / s, 2} \subset \Pi_{s / s, 2}
$$

satisfies $R\left(\psi^{A, b}\right)=2 s$, we have the following implications for each value of $s$.

$$
\text { Conjecture } 1.3 \text { with } m=n=s \Longrightarrow \text { Conjecture } 1.6 \Longrightarrow \text { Conjecture } 1.4
$$

and

Conjecture 1.3 with $m=n=s \Longrightarrow$ Conjecture $1.5 \Longrightarrow$ Conjecture 1.4.

As for the $p=3$ case, we pose the following new conjecture, which is stronger than $[\mathbf{3}$, Conjecture 3.2].

Conjecture 1.7. For each $s \geqslant 2, \widehat{R}_{s / s, 3}=s-1+\sqrt{s^{2}-1}$.

Conjectures 1.4-1.6 were previously proved only in the cases $s=1$ or $s=2$. The present work is devoted to determining the exact values of $R_{s / s, p}$ and $\widehat{R}_{s / s, p}$ for certain $2 \leqslant s \leqslant 4$ and $2 \leqslant p \leqslant 7$ pairs, as summarized in Tables 2.1 and 2.2. Our results lend some support to Conjectures 1.4 and 1.6, since we prove each of them for the cases $s=3$ (in $\S \S 2.4$ and 6.3) and $s=4$ (in $\S \S 2.4,7.4$ and A.2) $\dagger^{\dagger}$. We also prove Conjecture 1.7 for $2 \leqslant s \leqslant 4$ (the $s=2$ case is a direct consequence of the theorem in $\S 3$, while the $3 \leqslant s \leqslant 4$ cases are described in $\S \S 6.2$ and 7.3), simultaneously proving the $3 \leqslant s \leqslant 4$ special cases of [3, Conjecture 3.2].

The structure of the paper is as follows. In $\S 1.1$ we recall some theorems from $[\mathbf{5}, \mathbf{1 1}]$ on which our computations are based. The new results are summarized in $\S 2$, where we also provide some explanatory remarks. The proofs are given in $\S \S 3-7$. Section 2 has been written so that the casual reader need not refer to any later sections. Sections 2.4 and 2.5 provide an introduction to the structure and notation used in the proofs.

The proofs, comprising $\S \S 3-7$, proceed from smaller $s$ values to larger ones, and for each value of $s$, from larger $p$ values to smaller ones. Thus sections are ordered roughly in increasing difficulty within both the $\Pi_{s / s, p}$ and the $\widehat{\Pi}_{s / s, p}$ classes. These sections represent the fruits of several dozens of pages of computations, or of a few hundred pages, depending on the level of detail, so we had inevitably to omit some details of some proofs.

In $\S 8$, we give some auxiliary, but, in our opinion, related and interesting results that we could not (yet) tie to the main pieces of the puzzle (for example, to Conjecture 1.6), along with a few remarks about the successful (or failed) proof attempts and techniques.

In the Appendix, a few more algebraic expressions, mentioned only implicitly in the proofs, are collected to enable the reproducibility of certain longer computations.

Let us close this introduction with a remark explaining why the $p=1$ case is exceptional.

$\dagger$ Added in proof: in June 2013, an inductive proof for Conjecture 1.4 covering all $s \geqslant 1$ values was announced by Adrián Németh and Tihamér A. Kocsis. They believe that their technique can be extended to the DIRK class as well. However, a proof for the stronger Conjecture 1.6 for any $s \geqslant 5$ value is still out of reach. 
Remark 1.8 (On the $p=1$ case). For $\psi \in \Pi_{m / n, p}$ with $p \geqslant 2$, we have $R(\psi)<+\infty[\mathbf{1 2}]$. On the other hand, for $\psi(z)=1 /(1-z)$, we have $\psi \in \Pi_{0 / 1,1}$ and $R(\psi)=+\infty$. Consequently, we consider only the $p \geqslant 2$ case in this work.

\subsection{Some general results on the radius of absolute monotonicity}

Let us briefly summarize some useful theorems from $[\mathbf{5}, \mathbf{1 1}]$ that will frequently be used in this work. In [5], the following assumptions are made on the rational function $\psi$ :

(1) $\psi=P / Q$, where $P \in \Pi_{m}$ and $Q \in \Pi_{n}$ with some $m, n \in \mathbb{N}$, but $\psi$ is not a polynomial;

(2) $P$ and $Q$ have no common roots;

(3) $P(0)=Q(0)=1$.

REMARK 1.9. We will see that removing these assumptions (that is, not excluding removable singularities a priori, or considering, when setting up the form of the families of rational functions, the case $P(0)=Q(0)=0$ as well as interpreting $\psi(0)=1$ as $\lim _{0} \psi=1$ in the order conditions) does not make a difference in the optimal values $R_{s / s, p}$ and $\widehat{R}_{s / s, p}$ in the classes we are going to consider. Nevertheless, these assumptions are convenient in allowing us to immediately use results from [5].

Definition 1.10 [5, Definition 3.2]. Suppose that $\psi$ satisfies assumptions (1)-(3) above. Let $A^{+}(\psi)$ denote the set of poles of $\psi$ with non-negative imaginary part. For $\alpha \in A^{+}(\psi)$, we set

$$
I(\alpha):=\left\{x: x \in \mathbb{R} \text { and } \alpha \text { is the unique pole in } A^{+}(\psi) \text { closest to } x\right\} .
$$

The disjoint union of these intervals is the set $\mathbb{R}$ with only finitely many exceptions. Now we let

$$
B(\psi):= \begin{cases}-\inf I\left(\alpha_{0}\right) & \text { if } 0 \in I\left(\alpha_{0}\right) \text { for some positive real pole } \alpha_{0} \text { of } \psi \\ 0 & \text { otherwise }\end{cases}
$$

Note that $B(\psi)$ is determined solely by the location of the poles of $\psi$. The significance of this quantity is highlighted by the following theorem.

Theorem 1.11 [5, Theorem 3.3]. Suppose that $\psi$ satisfies assumptions (1)-(3) above. Then

$$
R(\psi) \leqslant B(\psi) .
$$

Theorem 1.12 [5, Corollary 3.4]. Suppose that $\psi$ satisfies assumptions (1)-(3) and $\psi$ has no positive real pole. Then $R(\psi)=0$.

Additionally, the negative real roots of the derivatives of $\psi$ form upper bounds on $R(\psi)$, as shown by the next theorem.

Theorem $1.13\left[5\right.$, Lemma 4.5]. Suppose that $\psi$ satisfies assumptions (1)-(3), and $\psi^{(\ell)}(x)=0$ for some $0 \geqslant x \in \mathbb{R}$ and $0 \leqslant \ell \in \mathbb{N}$. Then $R(\psi) \leqslant-x$.

We will also use the fact that under certain assumptions absolute monotonicity at the left endpoint of an interval implies absolute monotonicity on the whole interval.

Theorem 1.14 [11, Lemma 3.1]. Let $\psi=P / Q$ be absolutely monotonic at some $x<0$, where $P$ and $Q$ are polynomials and $Q$ has no zeros in $(x, 0]$. Then $R(\psi) \geqslant-x$. 
REMARK 1.15. In [5], formula (4.3) introduces an auxiliary quantity

$$
L(x)=\max (0, m-n+1, \max \{\ldots\})
$$

that is used in an algorithm to compute $R(\psi)$ for a given rational function. It may happen however that the $\{\ldots\}$ set above is empty (corresponding to the SDIRK case, for example), when a correct interpretation of this $\max \varnothing$ is $-\infty$ (or, say, 0$)$.

\section{Main results}

In $\S \S 2.1-2.3$ we state our main results about certain newly or exactly determined $R_{s / s, p}$ and $\widehat{R}_{s / s, p}$ values. In $\S 2.4$ we describe the two strategies we will follow in the proofs of these results; the proofs themselves are deferred to $\S \S 3-7$ and the Appendix.

\subsection{A lower bound on $R_{3 / 3,2}$}

In $\S 4.3$, we construct a function $\psi \in \Pi_{3 / 3,2}$ with $R(\psi)>6.7783>6$. This shows that Conjecture 1.3 does not hold for $m=n=3$. However, the exact value of $R_{3 / 3,2}$ is still unknown, because in order to describe all rational functions $\psi$ in $\Pi_{3 / 3,2}$, we need four parameters, rendering the (exact or numerical) optimization within this class impossible with our current techniques. In contrast, Theorem 2.2 in $\S 2.3$ asserts that $\widehat{R}_{3 / 3,2}=6$.

\subsection{The exact determination of certain $R_{s / s, p}$ values for $s \leqslant 4$}

2.2.1. Known exact values of $R_{s / s, p}$. Let us first review what is already known. The class $\Pi_{1 / 1, p}$ is empty for $p \geqslant 3$. The set $\Pi_{1 / 1,2}$ contains only the function $z \mapsto(1+z / 2) /(1-z / 2)$ with $R=2$, so $R_{1 / 1,2}=2$.

The class $\Pi_{2 / 2, p}$ is empty for $p \geqslant 5$. The set $\Pi_{2 / 2,4}$ consists of a single function $\psi$ : the $(2,2)$ Padé approximation to the exponential. This function has $\psi^{(6)}(0)=0$ (or, more precisely, for $k>0$ we have $\psi^{(k)}(0)=0$ if and only if $k$ is divisible by 6 ), so Theorem 1.13 implies $R_{2 / 2,4}=0$. More generally, each of the classes $\Pi_{s / s, p}$ with $p=2 s$ contains only the $(s, s)$ Padé approximation to the exponential. For $s$ even, [5, Theorem 5.1] shows via Theorem 1.12 that $R_{s / s, 2 s}=0$.

If $\psi=P / Q \in \Pi_{2 / 2,2}$, and $P$ and $Q$ have no common roots, then $[5, \S 6.3]$ says that $R(\psi) \leqslant 4$. If $P$ and $Q$ have a common root, then it is easily seen that $\psi \in \Pi_{1 / 1,2}$ also, hence $R(\psi) \leqslant 2$. For the function $\psi(z)=(1+z / 4)^{2} /(1-z / 4)^{2}$ we have $R(\psi)=4$, so $R_{2 / 2,2}=4$.

2.2.2. New exact values of $R_{s / s, p}$. For the cases in which $p=2 s$, a simple new result is the exact value of $R_{3 / 3,6}$. Its approximate value 2.2076 was presented in [5, Table 5.3].

As for the $p=2 s-1$ cases, $\Pi_{s / s, p}$ can be described by a 1-parameter family of rational functions. The exact value $R_{2 / 2,3}=1+\sqrt{3}$ was previously unknown: our computation confirms the corresponding numerical result 2.7320 given in [5, Table 6.1]. By using significantly more computational power, we also determined $R_{s / s, 2 s-1}$ for $s=3$ and $s=4$.

Decimal approximations of proven optimal $R_{s / s, p}$ values are summarized in Table 2.1, along with some other properties. Exact values are given in the sections indicated.

Combining the previously known results in $\S 2.2 .1$ and the new results in $\S 2.2 .2$, for $s \leqslant 4$, the exact $R_{s / s, p}$ values are now known for:

- $p \geqslant 2$ and $s=1,2$;

- $s=3,4$, and the corresponding $\Pi_{s / s, p}$ class can be described by at most one parameter. As for $s>4$ with $\Pi_{s / s, p} \neq \varnothing$, currently we have exact information on $R_{s / s, p}$ only when $s$ is even and $p=2 s$. 
REMARK 2.1 (On uniqueness). The optimal rational function in the $\Pi_{s / s, 2 s}$ class is unique, since $\Pi_{s / s, 2 s}$ has only one element. We prove in $\S \S 3-7$ that the optimal element in each of the $\Pi_{2 / 2,3}, \Pi_{3 / 3,5}$ and $\Pi_{4 / 4,7}$ classes is unique. Regarding the $\Pi_{2 / 2,2}$ class, it was not known previously whether there is only one element in $\Pi_{2 / 2,2}$ with $R=4$. We proved that $\psi \in \Pi_{2 / 2,2}$ and $R(\psi)=4$ imply that $\psi \in \widehat{\Pi}_{2 / 2,2}$ (the proof of this statement is not included in the present paper), but then Theorem 2.2 in $\S 2.3$ asserts that $\psi(z)=(1+z / 4)^{2} /(1-z / 4)^{2}$. Consequently, the optimal element is also unique in the $\Pi_{2 / 2,2}$ class.

\subsection{The exact determination of $\widehat{R}_{s / s, p}$ for $s \leqslant 4$}

Since the optimal elements of $\Pi_{1 / 1,2}$ and $\Pi_{2 / 2,2}, \psi(z)=(1+z / 2) /(1-z / 2)$ and $\psi(z)=$ $(1+z / 4)^{2} /(1-z / 4)^{2}$, given in $\S 2.2 .1$, are also elements of $\widehat{\Pi}_{1 / 1,2}$ and $\widehat{\Pi}_{2 / 2,2}$, respectively, we immediately have $\widehat{R}_{1 / 1,2}=R_{1 / 1,2}=2$ and $\widehat{R}_{2 / 2,2}=R_{2 / 2,2}=4$. Notice that these $\widehat{R}$ values are the $1 \leqslant s \leqslant 2$ special cases of Conjecture 1.6. On the other hand, uniqueness of the optimal element of $\widehat{\Pi}_{1 / 1,2}$ (being a singleton) is trivial, while uniqueness of the optimal element of $\widehat{\Pi}_{2 / 2,2}$ (a new result) will be shown in Theorem 2.2 .

As for the $\widehat{\Pi}_{2 / 2,3}$ class, it has only two elements:

$$
\psi_{231}(z):=\frac{-\frac{1}{6}(\sqrt{3}+1) z^{2}-z / \sqrt{3}+1}{\left(1-\frac{1}{6}(3+\sqrt{3}) z\right)^{2}}
$$

and

$$
\psi_{232}(z):=\frac{\frac{1}{6}(\sqrt{3}-1) z^{2}+z / \sqrt{3}+1}{\left(1-\frac{1}{6}(3-\sqrt{3}) z\right)^{2}}
$$

(the indexing of $\psi_{231}$ and $\psi_{232}$ is consistent with the notation described later in $\S 2.5$ ). It turns out that the optimal element of $\Pi_{2 / 2,3}$ (uniquely given by (3.1) in $\S 3$ ) is the same as $\psi_{232} \in \widehat{\Pi}_{2 / 2,3}$, hence $\widehat{R}_{2 / 2,3}=R_{2 / 2,3}=1+\sqrt{3}$.

The following theorem dealing with the $p=2$ case is of particular interest in light of the conjectures presented in $\S 1$.

TABLE 2.1. Optimal $R_{s / s, p}$ values, together with the algebraic degree of the optimal parameter choice within the given parametrization, the algebraic degree of $R_{s / s, p}$, the section number in which $R_{s / s, p}$ is given as an exact algebraic number, and the factor that limits the optimal value (see §1.1). Superscripts ${ }^{\dagger}$ and ${ }^{\ddagger}$ indicate, respectively, that the optimal $R_{s / s, p}$ value had already been proved exactly or had been presented numerically earlier. A - symbol means that the corresponding $\Pi_{s / s, p}$ class contains only finitely many elements hence the parameter $a$ is not present, whereas a + symbol denotes that the class cannot be described by only one parameter. In the $(s, p)=(2,4)$ case, $m$ is any positive integer. As for the last column, see Remark 2.3 as well.

\begin{tabular}{llcccr}
\hline$(s, p)$ & $R_{s / s, p}$ & $\operatorname{deg}\left(a^{*}\right)$ & $\operatorname{deg}\left(R_{s / s, p}\right)$ & $\operatorname{Section}(\mathrm{s})$ & Obstacle $\left(\right.$ with $\left.R:=R_{s / s, p}\right)$ \\
\hline$(2,2)$ & $=4^{\dagger}$ & + & 1 & 2.2 .1 & $\psi(-R)=\psi^{\prime}(-R)=0$ \\
$(2,3)$ & $\approx 2.732050^{\ddagger}$ & 2 & 2 & 3 & $\psi^{\prime}(-R)=0$ \\
$(2,4)$ & $=0^{\dagger}$ & - & 1 & 2.2 .1 & $R=B=0$ or $\psi^{(6 m)}(-R)=0$ \\
$(3,5)$ & $\approx 2.301322$ & 6 & 6 & 4.2 & $R=B$ \\
$(3,6)$ & $\approx 2.207606^{\ddagger}$ & - & 3 & 4.1 & $R=B$ \\
$(4,7)$ & $\approx 2.743911$ & 30 & 30 & 5 and A.1 & $R=B$ \\
$(4,8)$ & $=0^{\dagger}$ & - & 1 & 2.2 .1 & $R=B=0$ \\
\hline
\end{tabular}


Theorem 2.2. Fix $1 \leqslant s \leqslant 4$. Then $\widehat{R}_{s / s, 2}=2 s$, and the unique $\psi \in \widehat{\Pi}_{s / s, 2}$ that attains $R(\psi)=2 s$ is

$$
\psi(z)=\frac{(1+z /(2 s))^{s}}{(1-z /(2 s))^{s}}
$$

Let us highlight again that the $s=1$ case of this theorem is trivial, and in the $s=2$ case the value of $\widehat{R}_{2 / 2,2}=4$ was known previously; the new pieces of information are the $3 \leqslant s \leqslant 4$ cases and the uniqueness part for $s=2$. Theorem 2.2 confirms Conjecture 1.6 for $3 \leqslant s \leqslant 4$ (and extends it with a uniqueness part for $2 \leqslant s \leqslant 4$ ). It also proves Conjecture 3.1 in [3, $\S 3.2]$ for $3 \leqslant s \leqslant 4$ (the truth of this conjecture for $1 \leqslant s \leqslant 2$ being already established in [3]) regarding SDIRK methods that are optimal with respect to $R(A, b)$.

Finally, concerning some higher $p>2$ values, we determined $\widehat{R}_{3 / 3,3}, \widehat{R}_{3 / 3,4}, \widehat{R}_{4 / 4,3}, \widehat{R}_{4 / 4,4}$ and $\widehat{R}_{4 / 4,5}$ as exact algebraic numbers, and proved that the optimal element in each of the corresponding $\widehat{\Pi}_{s / s, p}$ classes is unique. The $\widehat{R}_{s / s, 3}$ values for $2 \leqslant s \leqslant 4$ support Conjecture 1.7, being stronger than [3, Conjecture 3.2]. In other words, we have proved [3, Conjecture 3.2] for $3 \leqslant s \leqslant 4$.

The known and newly obtained $\widehat{R}_{s / s, p}$ values are summarized in Table 2.2 . We also give some additional information on the structure of the optimal $\psi$ functions in Table 2.3.

As a corollary, the results of this section together with the fact that $\widehat{\Pi}_{s / s, p}=\varnothing$ for $1 \leqslant s \leqslant 4$ and $p \geqslant s+2$ imply that now we have a complete description of the optimal $\widehat{R}_{s / s, p}$ values for all $s \leqslant 4$ and $p \geqslant 2$.

REMARK 2.3. We have seen that the unique optimal rational functions corresponding to the $(s, p)=(2,2)$ and $(s, p)=(2,3)$ rows of Table 2.1 are also elements of the corresponding $\widehat{\Pi}_{s / s, p}$ classes. Moreover, it is easily checked that both of these optimal functions satisfy assumptions (1)-(3) given in $\S 1.1$, hence for these functions $B=+\infty$, and so $R<B$.

REMARK 2.4. On the one hand, according to (1.4), if $A$ and $b$ correspond to any SDIRK method of $s$ stages and order $p$, then we have $R(A, b) \leqslant \widehat{R}_{s / s, p}$. On the other hand, an SDIRK method is constructed in [3] for each pair $(s, p)$ satisfying $2 \leqslant s \leqslant 4$ and $2 \leqslant p \leqslant 3$ such that $R(A, b)=\widehat{R}_{s / s, p}$. Based on this, one might suspect that $\widehat{R}_{s / s, p}$ is equal to the optimal $R(A, b)$

TABLE 2.2. Optimal $\widehat{R}_{s / s, p}$ values, together with the algebraic degree of the optimal parameter value in the given parametrization and the algebraic degree of $\widehat{R}_{s / s, p}$, the section number in which they are given as exact algebraic numbers, and the first few derivatives of $\psi$ that vanish at $-\widehat{R}_{s / s, p}$ (see Theorem 1.13). The symbols ${ }^{\dagger},{ }^{\ddagger},+,-$ have the same meaning as in Table 2.1.

\begin{tabular}{llcccc}
\hline$(s, p)$ & $\widehat{R}_{s / s, p}$ & $\operatorname{deg}\left(a^{*}\right)$ & $\operatorname{deg}\left(\widehat{R}_{s / s, p}\right)$ & $\operatorname{Section}(\mathrm{s})$ & $\begin{array}{c}\text { Derivatives vanishing } \\
\text { at }-\widehat{R}_{s / s, p}\end{array}$ \\
\hline$(2,2)$ & $=4^{\dagger}$ & 1 & 1 & 2.3 & $\ell=0,1$ \\
$(3,2)$ & $=6$ & + & 1 & 2.4 and 6.3 & $\ell=0,1,2$ \\
$(4,2)$ & $=8$ & + & 1 & $2.4,7.4$ and $\mathrm{A} .2$ & $\ell=0,1,2,3$ \\
$(2,3)$ & $\approx 2.732050^{\ddagger}$ & - & 2 & 2.3 & $\ell=1$ \\
$(3,3)$ & $\approx 4.828427$ & 2 & 2 & 6.2 & $\ell=1,2$ \\
$(4,3)$ & $\approx 6.872983$ & + & 2 & 7.3 & $\ell=1,2,3$ \\
$(3,4)$ & $\approx 3.287278$ & - & 9 & 6.1 & $\ell=0$ \\
$(4,4)$ & $\approx 5.167265$ & 9 & 9 & 7.2 & $\ell=0,1$ \\
$(4,5)$ & $\approx 3.743299$ & - & 12 & 7.1 & $\ell=1$ \\
\hline & & & & &
\end{tabular}


radius for $s$-stage, order $p$ SDIRK methods for each $s, p \geqslant 2$. However, Remark 2.5 shows that this is not the case in general.

Remark 2.5. There exist exactly three functions in the set $\widehat{\Pi}_{3 / 3,4}$. These three rational functions are also mentioned in $[3, \S 3.4 .1]$. The method satisfying the non-negativity condition $K \geqslant 0$ in [3] is the one whose stability function yields the optimal $\widehat{R}_{3 / 3,4}$ value. This function has radius of absolute monotonicity $\widehat{R}_{3 / 3,4} \approx 3.2872$, but the corresponding optimal SDIRK method has only $R(A, b) \approx 1.7587$.

REMARK 2.6. The optimal $\psi$ in the $\widehat{\Pi}_{4 / 4,4}$ class is different from the stability function of the optimal method of $[3, \S 3.4 .2]$ in this class obtained by numerical search. In other words, according to the numerical tests in [3], the optimal $R(A, b)$ in the SDIRK $s=p=4$ case is $\approx 4.2081<\widehat{R}_{4 / 4,4}$ (cf. Remark 2.5).

\subsection{Introduction to the proofs in $\S \S 3-7$.}

2.4.1. Proofs in 1-parameter families with unknown optimum. This first approach will be followed in $\S \S 3,4.2,5,6.2$ and 7.2 , when there is only one parameter present, but the optimal radius of absolute monotonicity is unknown. In these cases, the method of proof we use is the following procedure (possibly in an iterative manner, because the number of derivatives to be checked in the first step below is also unknown in advance).

(1) Conjecture an optimal parameter value $a^{*}$ by inspection of the graphs of $a \mapsto B\left(\psi_{a}\right)$ or the first several derivatives of $\psi_{a}(\cdot)$.

(2) Rigorously exclude all the parameter values $\mathbb{R} \backslash\left\{a^{*}\right\}$ by appealing to, for example, Theorem 1.13 .

(3) Explicitly compute a formula for the $k$ th derivative ( $k \in \mathbb{N}$ arbitrary) and prove that $\left.\psi_{a^{*}}^{(k)}\right|_{\left[-x^{*}, 0\right]} \geqslant 0$, where $x^{*}>0$ is as large as possible due to step (1).

As a simple example of this technique, let us prove the $s=2$ case of Theorem 2.2. As mentioned in $\S 2.3$, this case was already known earlier apart from the uniqueness part.

Proof: First proof of Theorem 2.2 in the $s=2$ case. Any element of $\widehat{\Pi}_{2 / 2,2}$ can be represented as

$$
\psi_{a}(z)=\frac{\frac{1}{2}\left(2 a^{2}-4 a+1\right) z^{2}+(1-2 a) z+1}{(1-a z)^{2}}
$$

with $a \in \mathbb{R}$. First let us perform some preliminary reduction. For $a=0$, the rational function reduces to the second degree Taylor polynomial of the exponential function around 0, which

TABLE 2.3. Each row shows the appropriate derivative of the optimal element in $\widehat{\Pi}_{s / s, p}$ having multiple roots. In the $(s, p)=(4,4)$ case, approximate constants are used. In the $(s, p) \in\{(3,4)$, $(4,5)\}$ cases, no multiple roots were found among the first few derivatives of the optimal $\psi$ functions.

\begin{tabular}{rr}
\hline$(s, p)$ & Form of the optimal $\psi$ or its derivative \\
\hline$(2,2)$ & $\psi(z)=(1+z / 4)^{2} /(1-z / 4)^{2}$ \\
$(3,2)$ & $\psi(z)=(1+z / 6)^{3} /(1-z / 6)^{3}$ \\
$(4,2)$ & $\psi(z)=(1+z / 8)^{4} /(1-z / 8)^{4}$ \\
$(2,3)$ & $\psi^{\prime}(z)=\left(1+\frac{1}{2}\left(\sqrt{2^{2}-1}-1\right) z\right) /\left(1+\frac{1}{6}\left(\sqrt{2^{2}-1}-3\right) z\right)^{3}$ \\
$(3,3)$ & $\psi^{\prime}(z)=\left(1+\frac{1}{4}\left(\sqrt{3^{2}-1}-2\right) z\right)^{2} /\left(1+\frac{1}{8}\left(\sqrt{3^{2}-1}-4\right) z\right)^{4}$ \\
$(4,3)$ & $\psi^{\prime}(z)=\left(1+\frac{1}{6}\left(\sqrt{4^{2}-1}-3\right) z\right)^{3} /\left(1+\frac{1}{10}\left(\sqrt{4^{2}-1}-5\right) z\right)^{5}$ \\
$(4,4)$ & $\psi(z)=(1+0.193526 z)^{2}\left(1+0.224416 z+0.0437638 z^{2}\right) /(1-0.0971331 z)^{4}$ \\
\hline
\end{tabular}


has $R=1$. So we can assume $a \neq 0$. The numerator and denominator have a common root if and only if $a=\frac{1}{2}$, in which case $R=2$. For $a \neq \frac{1}{2}$, due to Theorem 1.12, $a>0$ is necessary to have $R>0$. So we can consider only the parameter values $0<a \neq \frac{1}{2}$.

For $a>\frac{3}{4}, \psi_{a}^{(4)}(0)=-12 a^{2}(4 a-3)<0$, implying $R=0$ here by definition. The optimal $\psi_{a}$, having the largest $R\left(=R_{\mathrm{opt}}\right)$, will be found when $a \in\left(0, \frac{3}{4}\right] \backslash\left\{\frac{1}{2}\right\}$. It turns out that $-R_{\mathrm{opt}}$ will be determined by the intersection of certain roots of $\psi_{a}$ and $\psi_{a}^{\prime}$. (Let us add that a plot of the roots of the lowest order derivatives of $\psi_{a}(\cdot)$ in the region $0<a \leqslant 3 / 4$ would look very similar to Figure 7.) For $a=1-\frac{1}{\sqrt{2}}$, the leading coefficient of the numerator of $\psi_{a}$ vanishes, and in this case $R \leqslant 1+\sqrt{2}$, because $\psi_{a}(x)<0$ for $x<-1-\sqrt{2}$. For $a \in\left(0, \frac{3}{4}\right] \backslash\left\{\frac{1}{2}, 1-\frac{1}{\sqrt{2}}\right\}$ and $a>\frac{1}{4}$, one negative root of $\psi_{a}(\cdot)$ is

$$
\frac{-1+2 a+\sqrt{4 a-1}}{2 a^{2}-4 a+1}>-4
$$

while for $a \in\left(0, \frac{3}{4}\right] \backslash\left\{\frac{1}{2}, 1-\frac{1}{\sqrt{2}}\right\}$ and $a<\frac{1}{4}$, the only root of $\psi_{a}^{\prime}(\cdot)$ is $0>1 /(3 a-1)>$ -4 , implying by Theorem 1.13 that for $a \neq \frac{1}{4}, R<4$. Finally, for $a=\frac{1}{4}, \psi_{a}(z)=$ $(1+z / 4)^{2} /(1-z / 4)^{2}$, and one can now directly check that $R=4$.

2.4.2. Proofs based on uniqueness and already conjectured optimal values. This is the approach we follow in $\S \S 6.3,7.3$ and 7.4. The strength of the argument is that we can handle classes that can be described by $\nu>1$ parameters; the disadvantage is, however, that the optimal value, $R_{\mathrm{opt}}>0$, must be conjectured in advance. With this preliminary information in hand, we consider the following infinite system of non-linear polynomial inequalities

$$
\psi^{(k)}\left(-R_{\mathrm{opt}}\right) \geqslant 0 \quad(k \geqslant 0)
$$

simply expressing absolute monotonicity of $\psi$ (depending on $\nu$ parameters in a non-linear way) at $x=-R_{\text {opt }}$. Then we show that the above system admits a unique solution in the $\nu$ parameters. Finally, we directly check that the corresponding optimal rational function, $\psi_{\text {opt }}$, is absolutely monotonic also on $\left(-R_{\text {opt }}, 0\right]$, but, by appealing to Theorem 1.13 , not absolutely monotonic on any larger interval $\left(-R_{\mathrm{opt}}-\varepsilon, 0\right]$ for any $\varepsilon>0$.

As a simple illustration, let us re-prove the $s=2$ case of Theorem 2.2 by using this technique.

Proof: Second proof of Theorem 2.2 in the $s=2$ case. Similarly to the first steps of the first proof, it is enough to consider only functions of the form $\psi_{a}(z)=\left(\frac{1}{2}\left(2 a^{2}-4 a+1\right)\right.$ $\left.z^{2}+(1-2 a) z+1\right) /(1-a z)^{2}$ with $a>0$, but now, as opposed to the first proof, we conjecture $\widehat{R}_{2 / 2,2}=4$ in advance. We show that

$$
\psi_{a}(-4) \geqslant 0, \quad \psi_{a}^{\prime}(-4) \geqslant 0
$$

and

$$
\forall k \in \mathbb{N}, k \geqslant 2: \quad \psi_{a}^{(k)}(-4) \geqslant 0
$$

imply $a=\frac{1}{4}$. Indeed, the first two conditions with $a>0$ amount to

$$
16 a^{2}-24 a+5 \geqslant 0 \text { and } 12 a-3 \geqslant 0
$$

that is, to $a \in\left\{\frac{1}{4}\right\} \cup\left[\frac{5}{4},+\infty\right)$. Then, instead of (2.4), it is enough to require a weaker property expressing the fact that the leading coefficient of a polynomial in $k$ has to be non-negative, if the polynomial is non-negative for all $k \geqslant 2$. This now simply reads as $\frac{1}{2}-a \geqslant 0$, implying the unique value $a=\frac{1}{4}$. 
In the rest of this section, we begin the proof of Theorem 2.2 in the $s=3$ and $s=4$ cases by performing some of the above-mentioned preliminary reductions.

For $s \geqslant 3$, one easily proves that any $\psi \in \widehat{\Pi}_{s / s, 2}$ can be written as

$$
\psi(z)=\frac{1+\left(1-a\left(\begin{array}{l}
s \\
1
\end{array}\right)\right) z+\left(\frac{1}{2}-a\left(\begin{array}{l}
s \\
1
\end{array}\right)+a^{2}\left(\begin{array}{l}
s \\
2
\end{array}\right)\right) z^{2}+\sum_{n=3}^{s} a_{n} z^{n}}{(1-a z)^{s}}
$$

with some $a, a_{3}, a_{4}, \ldots, a_{s} \in \mathbb{R}$. Let us denote the numerator of (2.5) by $P(z)$ and set $Q(z):=$ $1-a z$, so that $\psi=P / Q^{s}$. The next step is to exclude the non-positive $a$ values. Since Theorem 2.2 will be proved via the uniqueness argument

$$
R(\psi)=2 s \text { implies the unique form }(2.3) \text { of } \psi
$$

and $2 s>s-1$, the assumption of the lemma below is justified.

Lemma 2.7. Let $\psi \in \widehat{\Pi}_{s / s, 2}$ be given in the form (2.5), and suppose that $R(\psi)>s-1$. Then the parameter $a$ appearing in (2.5) satisfies $a>0$.

Proof. For $a=0, \psi$ is a polynomial approximating the exponential function to order $p=2$ near the origin. For such polynomials, $R(\psi) \leqslant s-1$ [10, Theorem 2.1]. For $a<0, \psi$ has no positive real poles, so we will apply Theorem 1.12 to show that $R(\psi)=0$. We need only to verify assumptions (1)-(3). Assumptions (1) and (3) are automatically satisfied by functions of the form (2.5) with $a<0$, so we are done if assumption (2) is also fulfilled. If not, then except at $z=1 / a$, $\psi$ can be expressed as $\widetilde{P} / \widetilde{Q}$, where $\widetilde{P}$ and $\widetilde{Q}$ have degree $\widetilde{s}_{P}<s$ and $\widetilde{s}_{Q}<s$, respectively, and no common roots. Clearly, $\widetilde{s}_{Q}>0$, since $p=2$ still holds. Moreover, $\widetilde{P}(0) / \widetilde{Q}(0)=P(0) / Q(0)=1$, so we can assume $\widetilde{P}(0)=\widetilde{Q}(0)=1$. Now application of Theorem 1.12 to $\widetilde{P} / \widetilde{Q}$ shows that $a<0$ implies $R(\psi)=0$.

Now we give the general form of the sign condition on the leading coefficient of the corresponding polynomial, mentioned in the second proof above. Assumption $a>0$ in the next lemma is guaranteed of course by the previous lemma.

Lemma 2.8. Suppose that $\psi=P / Q^{s}$ given by (2.5) with $a>0$ is absolutely monotonic at some $x<0$. Then

$$
\sum_{m=0}^{s} \frac{1}{m !}\left(\frac{1-a x}{a}\right)^{m} P^{(m)}(x) \geqslant 0
$$

Proof. From (2.5) it can be shown by induction that the $k$ th derivative $(k \in \mathbb{N})$ of $\psi$ is

$$
\psi^{(k)}=\left(\frac{P}{Q^{s}}\right)^{(k)}=\frac{\left(-Q^{\prime}\right)^{k} k !}{Q^{s+k}} \sum_{m=0}^{\min (k, s)} \frac{1}{m !}\left(\begin{array}{c}
s-1+k-m \\
s-1
\end{array}\right)\left(-\frac{Q}{Q^{\prime}}\right)^{m} P^{(m)} .
$$

Suppose that $\psi=P / Q^{s}$ is absolutely monotonic at some $x<0$. We now have $-Q^{\prime}(x)=a>0$ and $Q(x)=1-a x>0$, so (2.7) implies

$$
\lim _{k \rightarrow+\infty} \frac{1}{k^{s-1}} \sum_{m=0}^{\min (k, s)} \frac{1}{m !}\left(\begin{array}{c}
s-1+k-m \\
s-1
\end{array}\right)\left(\frac{1-a x}{a}\right)^{m} P^{(m)}(x) \geqslant 0 .
$$

By simplifying the binomial coefficients and multiplying both sides of the inequality by $(s-1)$ ! we easily see that the limit indeed exists and we obtain (2.6). 
After these preparations, the proof of Theorem 2.2 in the $s=3$ case is finished in $\S 6.3$, while the $s=4$ case is completed in Sections 7.4 and A.2. We show in both cases that (2.3) is the unique solution of the following infinite system of non-linear polynomial inequalities in the variables $a>0$ and $a_{3}, a_{4}, \ldots, a_{s} \in \mathbb{R}$ :

$$
\psi^{(k)}(-2 s) \geqslant 0 \quad \text { for } k=0,1, \ldots, s-1
$$

and

$$
\forall k \in \mathbb{N}, k \geqslant s: \quad \psi^{(k)}(-2 s) \geqslant 0 .
$$

The formal last step in both cases is given here: $R(\psi)$ for $(2.3)$ is indeed $2 \mathrm{~s}$.

\subsection{Notation used in the proof sections}

For $3 \leqslant n \in \mathbb{N}, n \geqslant m \in \mathbb{N}^{+}$and $a_{j} \in \mathbb{R}(j=0,1, \ldots, n)$ the $m$ th root of the polynomial equation $a_{n} z^{n}+a_{n-1} z^{n-1}+\ldots+a_{1} z+a_{0}=0$ will be denoted by

$$
\operatorname{root}_{m}\left(a_{n}, a_{n-1}, \ldots, a_{1}, a_{0}\right) \text {. }
$$

We use the following ordering for the roots: real roots are listed first in increasing order, then non-real complex roots follow as their real part increases, finally, complex roots with equal real part are sorted according to increasing imaginary part (in this context, multiple roots will not be encountered). So for example, the value of $\operatorname{rott}_{m}(1,0,0,0,0,0,-1)$ for $m=1,2, \ldots, 6$ is $-1,1,(-1-i \sqrt{3}) / 2,(-1+i \sqrt{3}) / 2,(1-i \sqrt{3}) / 2$ and $(1+i \sqrt{3}) / 2$, respectively, corresponding to the equation $z^{6}-1=0$. We will give numerical approximations to these roots as well, typically to six digits, if they occur only in auxiliary computations, but more digits will be displayed for significant constants. We will use the $\approx$ symbol without rounding, that is, in the sense that all shown digits are correct. However, we underline that these approximations are only given for the sake of the reader's convenience, and the proofs are not based upon them.

In our arguments, several exact algebraic numbers (typically denoted by $\varrho_{s p n}$ ), polynomials (denoted by $\left.P_{s p n}\right)$ or other auxiliary functions $\left(f_{s p n}\right)$ will appear. Here the subscripts $s$ and $p$ correspond to the ones in $\Pi_{s / s, p}$ or $\widehat{\Pi}_{s / s, p}$, whereas the positive integer $n$ serves as a counter within the actual section and is increased sequentially. Superscript * will often be used to denote optimal values within the family (for example, $a_{s p}^{*}$ being the parameter value corresponding to the rational function with maximal radius of absolute monotonicity in the class $\Pi_{s / s, p}$ or $\left.\widehat{\Pi}_{s / s, p}\right)$. If $\psi$ depends on a parameter, say, on $a$, then the $k$ th derivative $(k \in \mathbb{N})$ of $z \mapsto \psi_{a}(z)$ is denoted by $\psi_{a}^{(k)}$. Moreover, we will often use the simpler forms $R(a)$ and $B(a)$ instead of $R\left(\psi_{a}\right)$ and $B\left(\psi_{a}\right)$, based on the bijection $a \leftrightarrow \psi_{a}$.

REMARK 2.9. We note that the function $a \mapsto R(a) \in[0,+\infty]$ can be discontinuous. We will also see examples when the function $a \mapsto B(a) \in[0,+\infty]$ is non-differentiable, or convex on an interval and concave on another one.

\section{Determination of $R_{2 / 2,3}$}

TheOrem 3.1. We have $R_{2 / 2,3}=1+\sqrt{3}$, and if $\psi \in \Pi_{2 / 2,3}$ with $R(\psi)=R_{2 / 2,3}$, then $\psi=\psi_{a_{23}^{*}}$ given by (3.1).

Proof. The functions in $\Pi_{2 / 2,3}$ have the form

$$
\psi_{a}(z)=\frac{(1 / 3+a / 2) z^{2}+(a+1) z+1}{(-a / 2-1 / 6) z^{2}+a z+1},
$$




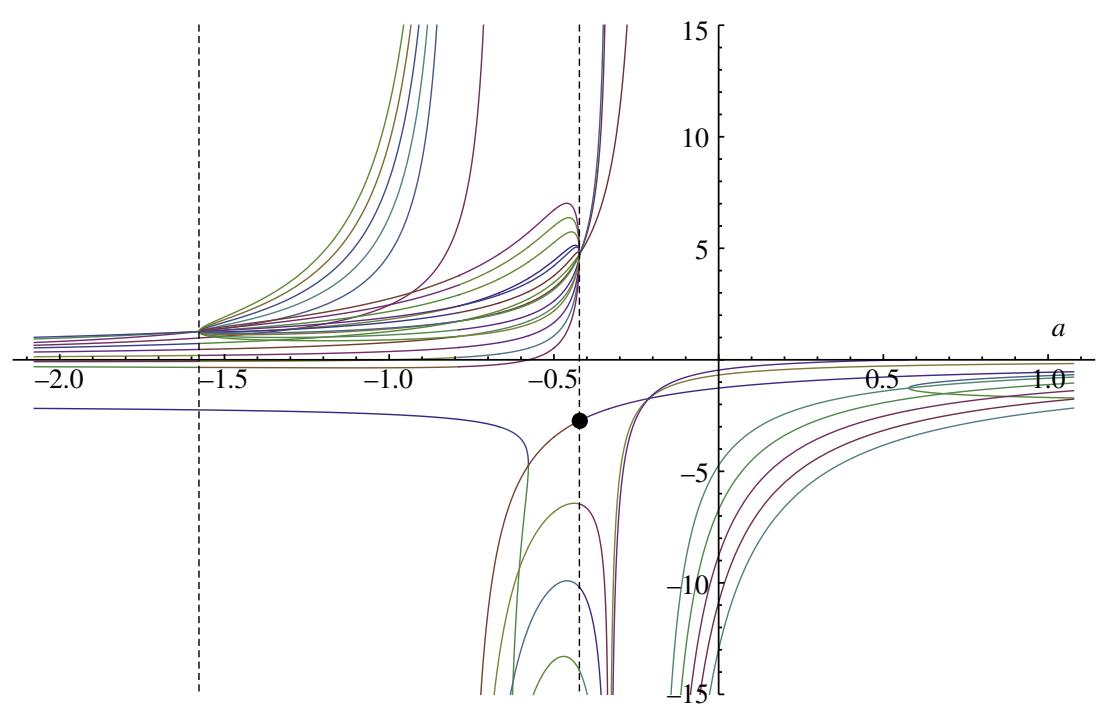

Figure 1. The figure shows roots of the derivatives $\psi_{a}^{(k)}(\cdot)$ as a function of $a$ for $0 \leqslant k \leqslant 5$ for the family of functions in the $\Pi_{2 / 2,3}$ class. The parameter region between the vertical dashed lines (that is, $-1-\frac{1}{\sqrt{3}}<a<-1+\frac{1}{\sqrt{3}}$ ) should be ignored, since here $R\left(\psi_{a}\right)=0$. The black dot indicates the optimal a parameter value and (the negative of) the optimal radius of absolute monotonicity within this class.

with $a \in \mathbb{R}$. For any $a \in \mathbb{R}$, the fraction cannot be simplified, since the resultant of the numerator and the denominator with respect to $z$ is $\frac{1}{12} \neq 0$. This implies that $\psi_{a}(\cdot)$ does not have removable singularities, only pole(s). This last statement remains valid if $\psi_{a}(\cdot)$ is replaced by its $k$ th derivative $(k \geqslant 1)$, since differentiation cannot introduce new removable or pole singularities, nor annihilate an existing pole. According to Theorem 1.12, $R\left(\psi_{a}\right)>0$ implies that $\psi_{a}$ has a positive real pole. This last condition holds if and only if $a \leqslant-1-\frac{1}{\sqrt{3}} \approx-1.57735$ or $a \geqslant-1+\frac{1}{\sqrt{3}} \approx-0.42264$. Figure 1 already suggests that the maximal radius of absolute monotonicity within this class is $1+\sqrt{3}$, corresponding to parameter value $a_{23}^{*}:=-1+\frac{1}{\sqrt{3}}$ (when the rational function has a pole of maximal possible order, that is, of order 2).

Indeed, for $a \leqslant-1-\frac{1}{\sqrt{3}}$, we have $\psi_{a}\left(-\frac{5}{2}\right)=-(15 a+14) /(135 a+1)<0$, so by definition $R\left(\psi_{a}\right) \leqslant \frac{5}{2}<1+\sqrt{3}$.

On the other hand, $\psi_{a}$ has a pole in $(-1-\sqrt{3}, 0)$ for $a>-1+\frac{5}{3 \sqrt{3}}>-1+\frac{1}{\sqrt{3}}$, meaning that $R\left(\psi_{a}\right)<1+\sqrt{3}$ here, since $\psi_{a}$ is not defined on the whole $(-1-\sqrt{3}, 0]$. Now we are going to exclude the parameter region $a \in\left(-1+\frac{1}{\sqrt{3}},-1+\frac{5}{3 \sqrt{3}}\right]$. For $a=-1+\frac{5}{3 \sqrt{3}}$, we have $R\left(\psi_{a}\right) \leqslant 2<1+\sqrt{3}$, because $\psi_{a}^{\prime}(-2)<0$, while for $a \in\left(-1+\frac{1}{\sqrt{3}},-1+\frac{5}{3 \sqrt{3}}\right)$, we have $R\left(\psi_{a}\right)<1+\sqrt{3}$ due to $\psi_{a}^{\prime}(-1-\sqrt{3})<0$.

Finally, we see that for $a=a_{23}^{*}$,

$$
\psi_{a_{23}^{*}}(x)=\frac{(2+\sqrt{3})\left((\sqrt{3}-1) x^{2}+2 \sqrt{3} x+6\right)}{(\sqrt{3}+3-x)^{2}},
$$

from which one proves recursively for $k \geqslant 1$ that

$$
\psi_{a_{23}^{*}}^{(k)}(x)=\frac{6 k !((12 k-3)+\sqrt{3}(7 k-2)+(3+2 \sqrt{3}) x)}{(\sqrt{3}+3-x)^{k+2}} .
$$


These imply that $\psi_{a_{23}^{*}}^{(k)}(x) \geqslant 0$ for all $k \geqslant 0$ and $-1-\sqrt{3} \leqslant x \leqslant 0$, but $\psi_{a_{23}^{*}}^{\prime}(x)<0$ for $x<-1-\sqrt{3}$, proving our claim.

4. Determination of $R_{3 / 3, p}$ for $5 \leqslant p \leqslant 6$ and a lower bound when $p=2$

\subsection{Determination of $R_{3 / 3,6}$}

The set $\Pi_{3 / 3,6}$ is a singleton, with the only element being

$$
\psi_{36}(z):=\frac{\left(z^{3} / 120\right)+\left(z^{2} / 10\right)+(z / 2)+1}{-\left(z^{3} / 120\right)+\left(z^{2} / 10\right)-(z / 2)+1} .
$$

Therefore $R_{3 / 3,6}=R\left(\psi_{36}\right)$.

The exact value of the above constant is given by the next theorem.

\section{THEOREM 4.1 .}

$$
R\left(\psi_{36}\right)=\operatorname{root}_{1}(1,-6,0,40)=-2+\sqrt[3]{4}(\sqrt[3]{3-\sqrt{5}}+\sqrt[3]{3+\sqrt{5}}) \approx 2.207606 .
$$

Proof. We first determine $B\left(\psi_{36}\right)$ (see Definition 1.10). Let $\alpha_{0}:=\operatorname{root}_{1}(1,-12,60,-120) \approx$ 4.64437 and $\alpha_{1}:=\operatorname{root}_{2}(1,-12,60,-120) \approx 3.67781-i \cdot 3.50876$ denote the real and one of the complex roots of the denominator of $\psi_{36}$. Then $x=x^{*}:=-B\left(\psi_{36}\right)<0$ is the unique real solution of the equation $\left|\alpha_{0}-x\right|=\left|\alpha_{1}-x\right|$. From this we see that $B\left(\psi_{36}\right)=\operatorname{root}_{1}(1,-6,0,40)$, and from Theorem 1.11 we know that $R\left(\psi_{36}\right) \leqslant B\left(\psi_{36}\right)$. In order to show that $R\left(\psi_{36}\right)=B\left(\psi_{36}\right)$, we verify that $\psi_{36}^{(k)}\left(x^{*}\right) \geqslant 0$ for any $k \geqslant 0$. (Then Theorem 1.14 with $x=x^{*}$ guarantees absolute monotonicity on $\left[-B\left(\psi_{36}\right), 0\right]$, since the denominator of $\psi_{36}$ does not vanish on, say, $(-\infty, 0]$.) The partial fraction decomposition of $\psi_{36}$ is $\psi_{36}(x)=$ $-1+c_{0} /\left(\alpha_{0}-x\right)+c_{1} /\left(\alpha_{1}-x\right)+\overline{c_{1}} /\left(\overline{\alpha_{1}}-x\right)$, with $c_{0}:=\operatorname{root}_{1}(1,-24,-1200,-40000) \approx$ 57.2025 and $c_{1}:=\operatorname{root}_{3}(1,-24,-1200,-40000) \approx-16.6012+i \cdot 20.5831$. But $\psi_{36}\left(x^{*}\right)=$ $\operatorname{root}_{1}(1,-15,2127,-233)>0$, and for $k \geqslant 1$ we have

$$
\psi_{36}^{(k)}\left(x^{*}\right)=k !\left(\alpha_{0}-x^{*}\right)^{-k-1}\left(c_{0}+c_{1}\left(\frac{\alpha_{0}-x^{*}}{\alpha_{1}-x^{*}}\right)^{k+1}+\overline{c_{1}}\left(\frac{\alpha_{0}-x^{*}}{\overline{\alpha_{1}}-x^{*}}\right)^{k+1}\right) .
$$

Since $\left|\left(\alpha_{0}-x^{*}\right) /\left(\alpha_{1}-x^{*}\right)\right|=\left|\left(\alpha_{0}-x^{*}\right) /\left(\overline{\alpha_{1}}-x^{*}\right)\right|=1$ by construction and $52.8874 \approx\left|c_{1}\right|+$ $\left|\overline{c_{1}}\right|<c_{0}$, the positivity of $\psi_{36}^{(k)}\left(x^{*}\right)$ follows.

\subsection{Determination of $R_{3 / 3,5}$}

ThEOREM 4.2. We have $R_{3 / 3,5}=B\left(a_{35}^{*}\right) \approx 2.301322$ given by (4.4), and if $\psi \in \Pi_{3 / 3,5}$ with $R(\psi)=R_{3 / 3,5}$, then $\psi$ is equal to (4.1) with $a=a_{35}^{*}$ given by (4.3).

Proof. The elements of $\Pi_{3 / 3,5}$ can be described by one real parameter $a \in \mathbb{R}$ as

$$
\psi_{a}(z)=\frac{\left(a+\frac{1}{60}\right) z^{3}+\left(6 a+\frac{3}{20}\right) z^{2}+\left(12 a+\frac{3}{5}\right) z+1}{a z^{3}+\left(\frac{1}{20}-6 a\right) z^{2}+\left(12 a-\frac{2}{5}\right) z+1} .
$$

The numerator and denominator do not have a common root for any $a$, because their resultant with respect to $z$ is $\frac{1}{8640} \neq 0$. 
Theorem 1.12 tells us that if $R\left(\psi_{a}\right)>0$, then $\psi_{a}$ has at least one positive real pole. We claim that the statement there exists $x>0: a x^{3}+\left(\frac{1}{20}-6 a\right) x^{2}+\left(12 a-\frac{2}{5}\right) x+1=0$ implies $a<0$. Indeed, after a rearrangement we get

$$
a=\frac{-x^{2}+8 x-20}{20 x\left(x^{2}-6 x+12\right)}<0,
$$

since the numerator is negative and the denominator is positive for all $x>0$.

Next we prove that $\psi_{a}^{(10)}(-2)<0$ for $a \leqslant-\frac{1}{50}$ : we have $\psi_{a}^{(10)}(-2)=P_{351}(a) \cdot(-189)$. $250000000^{-1}(28 a-1)^{-11}$, where $P_{351}$ is a polynomial of degree 11. Clearly, it is enough to show that $P_{351}(a)<0$ for $a \leqslant-\frac{1}{50}$. But $P_{351}^{(11)}$ is a positive constant, and for $k=10,9,8, \ldots, 0$ one computes that $\operatorname{sgn}\left(P_{351}^{(k)}\left(-\frac{1}{50}\right)\right)=(-1)^{k+1}$, showing inductively that for any $a \in\left(-\infty,-\frac{1}{50}\right]$ and $k=10,9,8, \ldots, 0$ we have $\operatorname{sgn}\left(P_{351}^{(k)}(a)\right)=(-1)^{k+1}$.

By definition, $\psi_{a}^{(10)}(-2)<0$ means that $R\left(\psi_{a}\right) \leqslant 2$ for $a \leqslant-\frac{1}{50}$. So in order to get $R\left(\psi_{a}\right)>2$, it is enough to consider $a \in\left(-\frac{1}{50}, 0\right)$. As it turns out, the maximal radius of absolute monotonicity for these rational functions will be determined by the (finite) maximum of the function $a \mapsto B(a) \equiv B\left(\psi_{a}\right)$ according to Definition 1.10 and Theorem 1.11.

First we give the real and imaginary parts of the three poles (even for all $a<0$ ), but due to symmetry, it is enough to take into account only the closed upper half-plane. For $a<0$, the real pole is located at

$$
\varrho_{351}(a)=\operatorname{root}_{1}(20 a, 1-120 a, 240 a-8,20) .
$$

As for the other pole, first we set

$$
\varrho_{352}=\operatorname{root}_{1}(864000,43200,360,1)=-\frac{1}{120}(2+\sqrt[3]{2}+\sqrt[3]{4}) \approx-0.0403944 .
$$

Then we consider the denominator of $\psi_{a}$, substitute $z=x+i y$, separate the real and imaginary parts and solve the resulting system: for $\varrho_{352} \neq a<0$, the upper complex pole is found at

$$
\varrho_{353}(a)+\frac{i}{4 \sqrt{5|a|}} \sqrt{\frac{14400 a^{2}+1200 a+4-(240 a+1) \varrho_{353}(a)}{60 a\left(2-\varrho_{353}(a)\right)-1}}
$$

with

$$
\varrho_{353}(a)=\operatorname{root}_{1}\left(1600 a^{2},-9600 a^{2}+80 a, 19200 a^{2}-400 a+1,-14400 a^{2}+400 a-4\right),
$$

while for $a=\varrho_{352}$, the upper complex pole is located at $4-\sqrt[3]{4}+i(2 \sqrt[3]{2}+\sqrt[3]{4}-2) \approx$ $2.4126+i \cdot 2.10724$. A case separation is necessary in the above formula to avoid a $0 / 0$ under the square root: geometrically this $a=\varrho_{352}$ value corresponds to the case when the three poles have the same real part.

Now with the real and imaginary parts separated, it is easy to find, for any $a<0$ the unique point on the real axis being equidistant from the real pole and the upper complex pole. We check that for $a<\varrho_{352}$ the (positive) real pole is strictly smaller than the real part of the (upper) complex pole, hence, by applying the notation of Definition 1.10 with $\alpha_{0} \equiv \alpha_{0}(a)$ being the positive real pole, the equidistant point on the real axis is strictly positive, meaning that $0 \in I\left(\alpha_{0}(a)\right)$ and $B(a)=-\inf I\left(\alpha_{0}(a)\right)=+\infty$ here. We easily see that $B\left(\varrho_{352}\right)=+\infty$ also, but for $\varrho_{352}<a<0, B(a)$ will be finite. For $\varrho_{352}<a<0$, the point on the real axis equidistant from the real pole and the complex pole is found at $f_{351}(a) / f_{352}(a)$ with

$$
\begin{aligned}
f_{351}(a):= & \varrho_{351}^{2}(a)\left(60 a \varrho_{353}(a)-120 a+1\right)-80 a \varrho_{353}^{3}(a) \\
& -(2-240 a) \varrho_{353}^{2}(a)-(240 a-8) \varrho_{353}(a)-20
\end{aligned}
$$




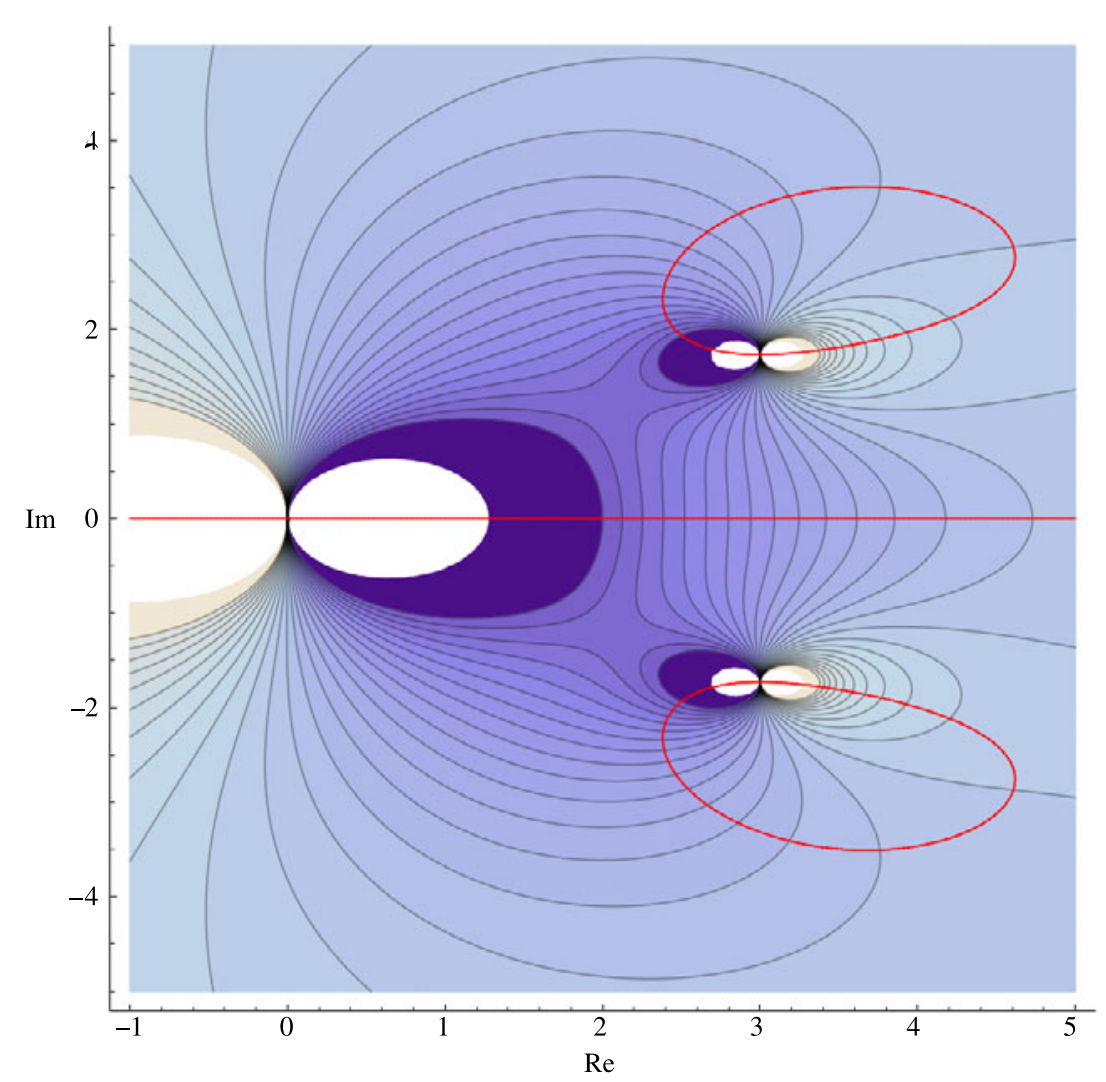

FiguRe 2. By substituting $z=x+i y(x, y \in \mathbb{R})$ into the fraction in (4.2) and separating real and imaginary parts, two $\mathbb{R}^{2} \rightarrow \mathbb{R}$ surfaces $\left(S_{\text {re }}\right.$ and $\left.S_{\text {im }}\right)$ are obtained. The contour $S_{\text {im }}=0$ yields the equation $y\left(x^{4}-16 x^{3}+2 x^{2} y^{2}+96 x^{2}-16 x y^{2}-240 x+y^{4}+16 y^{2}+240\right)=0$ depicted as red curves (with three connected components). They describe the locus of the poles of $\psi_{a}$ for $a \in \mathbb{R}$ (including the limiting case $a= \pm \infty$ ) in the complex plane in the $\Pi_{3 / 3,5}$ class. For a particular, fixed $a \in \mathbb{R}$, the three poles of $\psi_{a}$ are found when the red curves intersect the contour curves $S_{\text {re }}=a$ (some of which are shown as blue curves). The poles in the $a= \pm \infty$ limiting case are located at the three singular points (squeezed in between the white regions), where $S_{\text {re }}$ and $S_{\text {im }}$ are undefined.

and

$$
f_{352}(a):=2\left(\varrho_{351}(a)-\varrho_{353}(a)\right)\left(60 a \varrho_{353}(a)-120 a+1\right) .
$$

Now Reduce establishes that $f_{351}(a) / f_{352}(a)$ has a unique zero in $\varrho_{352}<a<0$ : by defining

$$
\varrho_{354}=\operatorname{root}_{1}(13824000,345600,2880,-152,-1) \approx-0.00625485,
$$

it turns out that

$$
\left.\frac{f_{351}}{f_{352}}\right|_{\left(\varrho_{352}, \varrho_{354}\right)}<0,\left.\quad \frac{f_{351}}{f_{352}}\right|_{a=\varrho_{354}}=0,\left.\quad \frac{f_{351}}{f_{352}}\right|_{\left(\varrho_{354}, 0\right)}>0 .
$$

Knowing the sign of $f_{351}(a) / f_{352}(a)$ implies that the relation $0 \in I\left(\alpha_{0}(a)\right)$ on $a \in\left(\varrho_{352}, 0\right) \backslash$ $\left\{\varrho_{354}\right\}$ can hold only for $a \in\left(\varrho_{352}, \varrho_{354}\right)$, so

$$
B(a)=0 \quad \text { for } a \in\left[\varrho_{354}, 0\right),
$$

and

$$
B(a)=-\inf I\left(\alpha_{0}(a)\right)=-\frac{f_{351}(a)}{f_{352}(a)} \quad \text { for } a \in\left(\varrho_{352}, \varrho_{354}\right)
$$




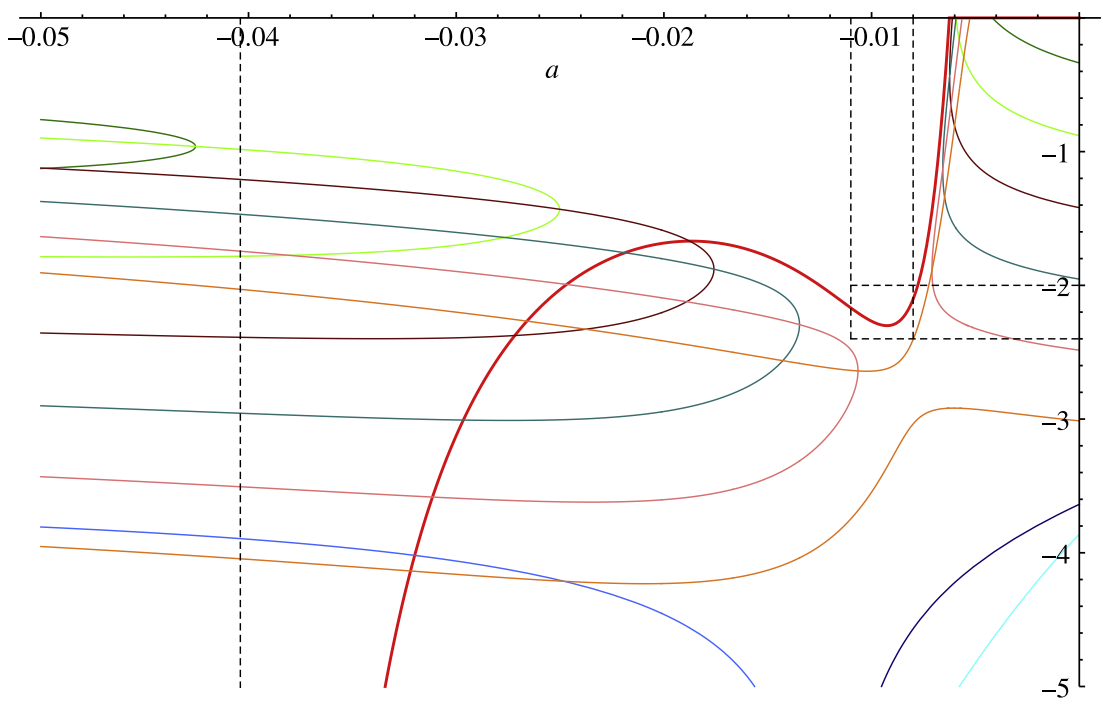

Figure 3 . In the $\Pi_{3 / 3,5}$ class the function $a \mapsto-B(a)$ is shown as the thick red curve (with $-B \equiv-\infty$ to the left of the long, vertical dashed line). The small dashed rectangle will contain the optimal value of $-B$. The other curves are the roots of the derivatives $\psi_{a}^{(k)}(\cdot)$ for $k=0,1,2,8,9,10,11,12,13$. In this plot window, these are the only roots of $\psi_{a}^{(k)}(\cdot)$ for $k \leqslant 13$. We note that altogether 17947 digits are needed just to write down the integer polynomials appearing in the numerators of $\psi_{a}^{(k)}(\cdot)$ for these $k$ values. The structures analogous to pitchfork bifurcations in this figure nicely illustrate Rolle's theorem, further, the fact that if a smooth function has a root of multiplicity $m$ at a point, then its derivative also has a root there (with multiplicity $m-1$ ).

Having completely described the function $B$, in the next step we will determine its maximal value on $a \in\left(-\frac{1}{50}, 0\right)$ by differentiation. We check by computing the discriminant of the denominator of $\psi_{a}$ that all roots of the denominator are simple for all $a<0$. Moreover, this denominator is smooth in $x$ and $a$, so the implicit function theorem yields that the functions $a \mapsto \varrho_{351}(a)$ and $a \mapsto \varrho_{353}(a)$ are differentiable with derivatives

$$
\varrho_{351}^{\prime}(a)=-\frac{20 \varrho_{351}^{3}(a)-120 \varrho_{351}^{2}(a)+240 \varrho_{351}(a)}{60 a \varrho_{351}^{2}(a)+2(1-120 a) \varrho_{351}(a)+240 a-8}
$$

and

$$
\varrho_{353}^{\prime}(a)=-\frac{3200 a \varrho_{353}^{3}(a)+(-19200 a+80) \varrho_{353}^{2}(a)+(38400 a-400) \varrho_{353}(a)-28800 a+400}{4800 a^{2} \varrho_{353}^{2}(a)+2\left(-9600 a^{2}+80 a\right) \varrho_{353}(a)+19200 a^{2}-400 a+1} .
$$

Also using these formulae we determine that there are exactly two $a$ values in $\left(-\frac{1}{50}, 0\right)$ such that $B^{\prime}(a)=0$; one is a local minimum and the other one is a local maximum of $B$. But since we are maximizing $B$, the local minimum is ignored. The local maximum of $B$ is located at

$$
\begin{aligned}
& a_{35}^{*} \\
& \quad:=\operatorname{root}_{2}(13436928000000,1492992000000,68428800000,1427328000,13867200,61200,101) \\
& \quad \approx-0.009257142292762484937472363
\end{aligned}
$$

with value

$$
B\left(a_{35}^{*}\right)=\operatorname{root}_{2}(1,12,36,-76,-360,0,900) \approx 2.301322934003485801187482 .
$$


Note that Figure 3 depicts $-B$ so that it can be compared with the derivatives of $\psi_{a}(\cdot)$. We note that the local maximum of $-\left.B\right|_{\left(-\frac{1}{50}, 0\right)}$ is described by the same root object as $a_{35}^{*}$ but with root $_{2}$ replaced by root $_{1}$.

As a final step, we show that the interval $\left[-B\left(a_{35}^{*}\right), 0\right]$ does not contain any roots of the derivatives $\psi_{a_{35}^{*}}^{(k)}(\cdot)(k=0,1, \ldots)$. In view of Theorem 1.14 with $x=-B\left(a_{35}^{*}\right)$, it is enough to show that for all $k \geqslant 0$ we have

$$
\psi_{a_{35}^{*}}^{(k)}\left(-B\left(a_{35}^{*}\right)\right) \geqslant 0,
$$

which we check by partial fraction decomposition (also showing that there are no poles of $\psi_{a_{35}^{*}}$ in $\left[-B\left(a_{35}^{*}\right), 0\right]$, so Theorem 1.14 applies). The function $\psi_{a_{35}^{*}}$ evaluated at $x$ admits the decomposition

$$
c+\frac{c_{0}}{\alpha_{0}-x}+\frac{c_{1}}{\alpha_{1}-x}+\frac{\overline{c_{1}}}{\overline{\alpha_{1}}-x}
$$

with some $c, c_{0} \in \mathbb{R}, \alpha_{0}>0$ and $c_{1}, \alpha_{1} \in \mathbb{C} \backslash \mathbb{R}$. These constants are algebraic numbers of degree not exceeding 12, and absolute value of the coefficients of their defining integer polynomials less than $10^{26}$. Here we give only their numerical approximations as

$$
\begin{gathered}
c \approx-0.800411, \quad c_{0} \approx 46.829419, \quad \alpha_{0} \approx 4.449434, \\
c_{1} \approx-13.750016-i \cdot 16.640148, \quad \alpha_{1} \approx 3.475900+i \cdot 3.492336 .
\end{gathered}
$$

With $x=x^{*}:=-B\left(a_{35}^{*}\right)<0$ we check that

$$
0.09999997 \approx \psi_{a_{35}^{*}}\left(x^{*}\right)>0 .
$$

On the other hand, for $k \geqslant 1, \psi_{a_{35}^{*}}^{(k)}\left(x^{*}\right)$ takes the form

$$
k !\left(\alpha_{0}-x^{*}\right)^{-k-1}\left(c_{0}+c_{1}\left(\frac{\alpha_{0}-x^{*}}{\alpha_{1}-x^{*}}\right)^{k+1}+\overline{c_{1}}\left(\frac{\alpha_{0}-x^{*}}{\overline{\alpha_{1}}-x^{*}}\right)^{k+1}\right) .
$$

Now we make use of the facts that $\alpha_{0}-x^{*}>0$ and $\left|\left(\alpha_{0}-x^{*}\right) /\left(\alpha_{1}-x^{*}\right)\right|=\mid\left(\alpha_{0}-x^{*}\right) /$ $\left(\overline{\alpha_{1}}-x^{*}\right) \mid=1$ by construction, further, that $c_{0}>0$ and $43.172096 \approx\left|c_{1}\right|+\left|\overline{c_{1}}\right|<c_{0}$. This proves that $\psi_{a_{35}^{*}}^{(k)}\left(x^{*}\right)>0$ also for $k \geqslant 1$.

\subsection{A counterexample to the conjecture that $R_{s / s, 2}=2 s$}

A counterexample to Conjecture 1.3 for the case $m=n=3$ was found via extensive numerical search using MATLAB and the Symbolic Toolbox employing the fminsearch function (NelderMead simplex method); it originally appeared in [8]. For the sake of our presentation, let us denote this counterexample with rational coefficients by $\widetilde{\psi}_{32}{ }^{\dagger}$. Now we are going to present a simpler counterexample, in the sense that the rational coefficients have much smaller numerators and denominators, with slightly improved radius of absolute monotonicity.

By suitably perturbing the rational coefficients into nearby simpler ones and embedding $\widetilde{\psi}_{32}$ into a one-parameter family of rational functions in a way that the order conditions are satisfied within the family, say, as

$$
\psi_{c}(z)=\frac{c z^{3}+\frac{2289}{34970} z^{2}+\frac{119}{269} z+1}{-\frac{4}{327} z^{3}+\frac{8}{65} z^{2}-\frac{150}{269} z+1}
$$

with $c \in \mathbb{R}$, we can optimize the radius of absolute monotonicity with respect to $c$ using Mathematica. It turns out that the optimal parameter $c^{*}$ (that is, the one that yields the

\footnotetext{
†We note that there is a typo in the last digit of one of its coefficients in [8], instead of 7969150767159903, one should have 7969150767159904 .
} 
maximal $R$ within this chosen class) is an algebraic number of degree 5 . By replacing $c^{*}$ with a nearby simple rational number we get, for example,

$$
\psi_{32}(z)=\frac{\frac{1246}{384649} z^{3}+\frac{2289}{34970} z^{2}+\frac{119}{269} z+1}{-\frac{4}{327} z^{3}+\frac{8}{65} z^{2}-\frac{150}{269} z+1} .
$$

This function has

$$
\begin{aligned}
R\left(\psi_{32}\right) & =\operatorname{root}_{1}(43572620,-880461561,5950520030,-13451175530) \\
& \approx 6.778307398562974637718719>6 .
\end{aligned}
$$

We note that $6.77823595 \approx R\left(\widetilde{\psi}_{32}\right)<R\left(\psi_{32}\right)<R\left(\psi_{c^{*}}\right) \approx 6.77830907$.

Let us briefly give some details that can be used to verify the above value of $R\left(\psi_{32}\right)$. The function $\psi_{32}$ at $x$ has the partial fraction decomposition

$$
c+\frac{c_{0}}{\alpha_{0}-x}+\frac{c_{1}}{\alpha_{1}-x}+\frac{\overline{c_{1}}}{\overline{\alpha_{1}}-x}
$$

with $c=-\frac{203721}{769298}, c_{0} \approx 24.8122, \alpha_{0} \approx 3.71417, c_{1} \approx-8.39838-i \cdot 9.53528$ and $\alpha_{1} \approx 3.17368+$ $i \cdot 3.45514$. (These are all algebraic numbers of degree 3 , and with maximal absolute value of the coefficients in their defining integer polynomials approximately $1.046 \cdot 10^{42}$.) We see that $\psi_{32}$ has a unique real root at $x^{*}:=-R\left(\psi_{32}\right) \approx-6.778307$, so Theorem 1.13 with $x=x^{*}$ and $\ell=0$ applies. We claim that $\psi_{32}^{(k)}\left(x^{*}\right) \geqslant 0$ holds for all $k \geqslant 1$ as well. For $k \geqslant 1$, we have

$$
\psi_{32}^{(k)}\left(x^{*}\right)=k !\left(\alpha_{0}-x^{*}\right)^{-k-1}\left(c_{0}+c_{1}\left(\frac{\alpha_{0}-x^{*}}{\alpha_{1}-x^{*}}\right)^{k+1}+\overline{c_{1}}\left(\frac{\alpha_{0}-x^{*}}{\overline{\alpha_{1}}-x^{*}}\right)^{k+1}\right) .
$$

Now since $\alpha_{0}-x^{*}>0$ and $\left|\left(\alpha_{0}-x^{*}\right) /\left(\alpha_{1}-x^{*}\right)\right|=\left|\left(\alpha_{0}-x^{*}\right) /\left(\overline{\alpha_{1}}-x^{*}\right)\right| \approx 0.995991$ (an algebraic number of degree 18 , with 92 -digit integers as coefficients), we see that the sufficient condition

$$
2\left|c_{1}\right|\left|\frac{\alpha_{0}-x^{*}}{\alpha_{1}-x^{*}}\right|^{k+1} \leqslant c_{0}
$$

for the non-negativity of $\psi_{32}^{(k)}\left(x^{*}\right)$ holds for $k \geqslant 5$. Finally, we directly check that $\psi_{32}^{(k)}\left(x^{*}\right)>0$ is also valid for $1 \leqslant k \leqslant 4$. (We note that now $B\left(\psi_{32}\right) \approx 7.59982$ is an algebraic number of degree 3 , so for this particular function $R\left(\psi_{32}\right)<B\left(\psi_{32}\right)$.) Absolute monotonicity in the whole $\left[x^{*}, 0\right]$ interval is guaranteed by Theorem 1.14 , by taking into account that there are no poles of $\psi_{32}$ in $\left[x^{*}, 0\right]$.

Of course, it is to be emphasized that the maximal radius of absolute monotonicity in the whole $\Pi_{3 / 3,2}$ class is still unknown: this class of rational functions can be described by four parameters. It is a major open challenge to find the maximal $R$ within this 4 -parameter family.

\section{Determination of $R_{4 / 4,7}$}

Theorem 5.1. We have $R_{4 / 4,7}=B\left(a_{47}^{*}\right) \approx 2.743911$, and if $\psi \in \Pi_{4 / 4,7}$ with $R(\psi)=R_{4 / 4,7}$, then $\psi$ is equal to (5.1) with $a=a_{47}^{*}$. The exact values of $a_{47}^{*}$ and $B\left(a_{47}^{*}\right)$ are found in $\S$ A.1.

Proof. Elements of the set $\Pi_{4 / 4,7}$ can be described by one real parameter $a \in \mathbb{R}$ as

$$
\psi_{a}(z)=\frac{\frac{1}{840}(7 a+4) z^{4}+\frac{1}{210}(21 a+13) z^{3}+\left(-\frac{1}{14}(7 a+2)+a+\frac{1}{2}\right) z^{2}+(a+1) z+1}{-\frac{1}{840}(7 a+3) z^{4}+\frac{1}{210}(21 a+8) z^{3}-\frac{1}{14}(7 a+2) z^{2}+a z+1} .
$$


The numerator and denominator do not have common factors for any $a$, since their resultant with respect to $z$ is $\frac{1}{870912000} \neq 0$. In order to have $R\left(\psi_{a}\right)>0, \psi_{a}$ needs to have at least one positive real pole (Theorem 1.12). To obtain some preliminary information on the location of the real poles of $x \mapsto \psi_{a}(x)$, we apply the same trick as at the beginning of $\S 4.2$. This time the denominator of $\psi_{a}(x)$ (with $x \in \mathbb{R}$ ) vanishes if and only if

$$
a=\frac{-3 x^{4}+32 x^{3}-120 x^{2}+840}{7 x\left(x^{3}-12 x^{2}+60 x-120\right)} .
$$

Then, by analyzing the range of this rational function on the right-hand side (under the restriction $x>0$ ), we prove that $\psi_{a}$ has at least one positive real pole precisely if

$$
a \leqslant \varrho_{471} \approx-0.843194 \text { or } a \geqslant \varrho_{472} \approx-0.471357,
$$

so in the rest of the proof we can consider only the above parameter set. We note that the exact values of these and the following algebraic numbers are listed in the Appendix.

Now we show that in the left unbounded component, that is for $a \in\left(-\infty, \varrho_{471}\right]$, we have $R(a) \equiv R\left(\psi_{a}\right)=0$. Indeed, $a \in\left(-\infty, \varrho_{471}\right] \subset\left(-\infty,-\sqrt{\frac{11}{21}}\right)$ implies $\psi_{a}^{(9)}(0)=\frac{66}{35}-\frac{18 a^{2}}{5}<0$.

Hence it is enough to focus our attention on the right unbounded component $a \in\left[\varrho_{472},+\infty\right)$. In this region we are going to explicitly compute the function $a \mapsto B(a) \equiv B\left(\psi_{a}\right)$ (according to Definition 1.10).

As a first step, Reduce was able to give a complete description of the locus of the poles in the complex plane with real and imaginary parts separated as $a$ is varied. For $a=\varrho_{472}$ we have a real pole of order 2 at $\varrho_{473} \approx 7.64527$. For $\varrho_{472}<a<-\frac{3}{7}$, it bifurcates into two real poles (of order 1) located at $\varrho_{4711}(a) \in\left(\varrho_{474}, \varrho_{473}\right)$, and $\varrho_{4712}(a) \in\left(\varrho_{473},+\infty\right)$, with $\varrho_{474} \approx 5.64849$. For $a=-\frac{3}{7}$ there is a unique real pole of order 1 at $\varrho_{474}$. Finally, for $a>-\frac{3}{7}$ the smaller (negative) real pole is located at $\varrho_{4711}(a)<0$, while the larger (positive) pole is found at $\varrho_{4712}(a)$.

As for the complex poles, due to symmetry, it is enough to describe only the one with positive imaginary part. For $a \in\left[\varrho_{472},-\frac{3}{7}\right]$, the complex pole in the upper half-plane has real part $\varrho_{477}(a)$ and imaginary part

$$
\sqrt{\frac{(7 a+3) \varrho_{477}^{3}(a)-(63 a+24) \varrho_{477}^{2}(a)+(210 a+60) \varrho_{477}(a)-210 a}{(7 a+3) \varrho_{477}(a)-21 a-8}} .
$$

For $a>-\frac{3}{7}$ the expressions for the real and the imaginary parts of the upper complex pole are obtained as above, but with $\operatorname{root}_{1}$ in the definition of $\varrho_{477}(a)$ (see the Appendix) replaced by $\operatorname{root}_{2}$.

Figure 4 depicts the locus of the poles of $\psi_{a}$ as $a$ is varied. For the sake of completeness, we have also included the poles corresponding to the interval $a \in\left(-\infty, \varrho_{472}\right)$.

Now we combine the above formulae on the locus of the poles, real and imaginary parts separated, to determine $B(a)$ for each $a \in\left[\varrho_{472},+\infty\right)$. With the help of Reduce (and by applying some geometric reformulations to be able to obtain the results in a reasonable amount of computing time, but now omitting the details here) we can prove that for $-0.471357 \approx$ $\varrho_{472} \leqslant a<\varrho_{478} \approx-0.469514$ the point on the real axis equidistant from the smaller (positive) real pole and the upper complex pole is positive (that is, 0 lies strictly closer to the nonreal upper complex pole for $a \in\left[\varrho_{472}, \varrho_{478}\right)$ ), hence (in the sense of Definition 1.10) $0 \notin$ $I\left(\alpha_{0}\right)$, so $B(a)=0$ here. The larger real pole, existing for $a \in\left(\varrho_{472},-\frac{3}{7}\right)$, clearly cannot influence the value of $B(a)$ now. For $a \in\left[\varrho_{478},-\frac{3}{7}\right]$, the equidistant point on the real axis from the smaller positive real pole and the upper complex pole is non-positive, and is given 


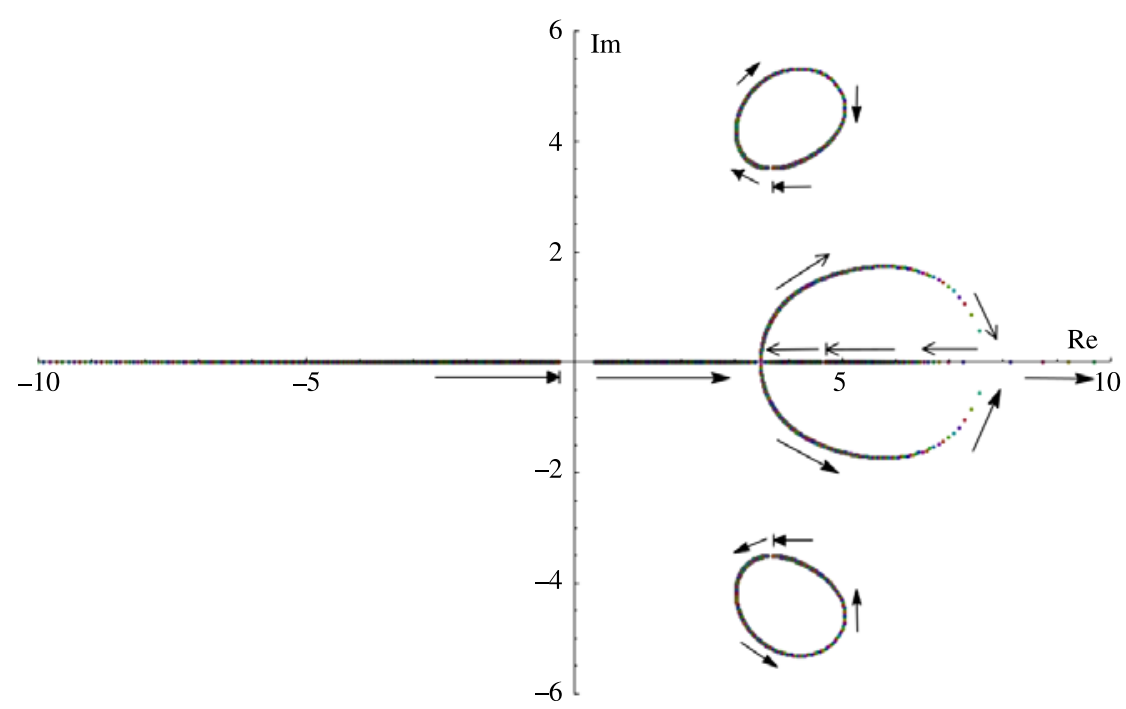

FiguRE 4 . The trajectory of the four poles of $\psi_{a}$ in the complex plane in the $\Pi_{4 / 4,7}$ class as parameter a traverses through the real line from $-\infty$ to $+\infty$. As $a \rightarrow-3 / 7^{-}$and passes through this point, the larger real pole is repelled to $+\infty$, then it enters the real line again from $-\infty$ and approaches to $0^{-}$. (In order to create the figure, we have used an equidistant grid on $a \in[-3,3]$ and Mathematica's NSolve approximated the roots of the resulting 6001 quartic equations appearing as denominators in a few seconds.) Compare these curves with the corresponding red curves in Figure 2.

by $f_{471}(a) / f_{472}(a)$ with

$$
\begin{aligned}
f_{471}(a):= & 2(7 a+3) \varrho_{477}^{3}(a)-4(21 a+8) \varrho_{477}^{2}(a)+30(7 a+2) \varrho_{477}(a) \\
& +\left(21 a+8-(7 a+3) \varrho_{477}(a)\right) \varrho_{4711}^{2}(a)-210 a
\end{aligned}
$$

and

$$
f_{472}(a):=2\left((7 a+3) \varrho_{477}(a)-21 a-8\right)\left(\varrho_{477}(a)-\varrho_{4711}(a)\right) .
$$

(Of course, as already noted earlier, the expression 'smaller positive real pole' in the previous sentence should be interpreted as the 'unique real pole' for $a=-\frac{3}{7}$. Moreover, the expression 'non-positive' can be replaced by 'zero' for $a=\varrho_{478}$, and by 'negative' for $a \in\left(\varrho_{478},-\frac{3}{7}\right]$.) Finally, we study the interval $a>-\frac{3}{7}$. Now one should take into account the newly created negative real pole as well. It can be proved that for $a=\varrho_{479} \approx-0.358565$ there is a unique negative real number, $\operatorname{root}_{1}(1,-21,165,-520,0,3600,-6000) \approx-2.40614$ such that it is equidistant from all the four poles. For $a \in\left(-\frac{3}{7}, \varrho_{479}\right]$ the negative real pole is still too far on the left to have an effect on $B(a)$, so for these $a$ values $B(a)$ is obtained as the absolute value of the point on the real axis equidistant from the positive real pole and the upper complex pole. Here $B(a)$ will be given in terms of $f_{473}(a)$ and $f_{474}(a)$. Expression $f_{473}(a)$ is defined just as $f_{471}(a)$, but with each root $_{1}$ occurring in the definition of $\varrho_{477}(a)$ replaced by $\operatorname{root}_{2}$, further, with $\varrho_{4711}(a)$ replaced by $\varrho_{4712}(a)$. Expression $f_{474}(a)$ is obtained from $f_{472}(a)$ via the same two replacement rules. For $a>\varrho_{479}$, the

(equidistant point on the real axis from the positive real pole and the upper complex pole) 1 is smaller than the

(equidistant point on the real axis from the positive real pole and the negative real pole) ${ }_{2}$, 
hence here $B(a)$ is determined solely by the two real poles. Notice that quantity $(\ldots)_{2}$ is simply the midpoint between the two real poles. For

$$
-0.358565 \approx \varrho_{479}<a \leqslant \varrho_{4710} \approx-0.274796,
$$

the expression $(\ldots)_{2}$ is non-positive, so its absolute value gives $B(a)$. But for $a>\varrho_{4710},(\ldots)_{2}$ is strictly positive, so $0 \notin I\left(\alpha_{0}\right)$ again, thus $B(a)=0$ here.

We now summarize the above information in one formula for $B(a)$. (For completeness' sake we also provide some additional information on $B(a)$ in the interval $a \in\left(-\infty, \varrho_{472}\right.$ ] already investigated earlier. We note that $a=\varrho_{475} \approx-0.850052$ corresponds to the geometric configuration when $\psi_{a}$ has three poles, one positive real and two complex poles, with equal real part, and the fourth, real pole lies to the right.) We have

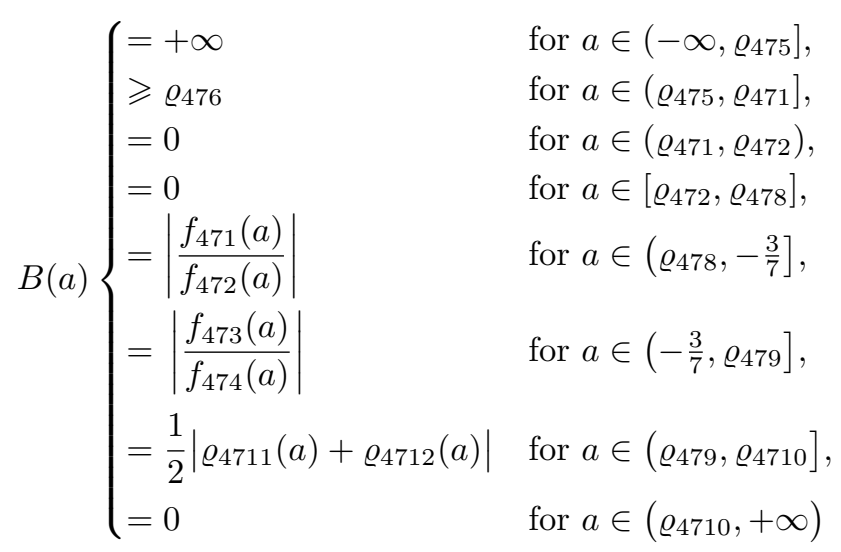

with $\varrho_{476} \approx 21.5907$.

After describing the function $B$, we find its maximal value on $a \in\left[\varrho_{472},+\infty\right)$, or, equivalently, on $\left[\varrho_{472}, \varrho_{4710}\right]$, see Figure 5. Mathematica's Maximize was able to locate this unique point. It turns out that $\left.B\right|_{\left[\varrho_{472}, \varrho_{4710}\right]}$ attains its maximal value at

$$
a_{47}^{*} \approx-0.4398493860002001824004494,
$$

where $a_{47}^{*}$ is the smaller real root of a polynomial with integer coefficients and of degree 30 . The corresponding maximal value is

$$
B\left(a_{47}^{*}\right) \approx 2.743911895676330804848228,
$$

expressible as the larger real root of a polynomial with integer coefficients and again of degree 30, see the Appendix.

As a last step, we check that no roots of the derivatives of the function $\psi_{a_{47}^{*}}(\cdot)$ can enter the interval $\left[-B\left(a_{47}^{*}\right), 0\right]$, so its radius of absolute monotonicity is as large as it can be (Theorem 1.11), based exclusively on the location of the poles: in view of Theorem 1.14 with $x=-B\left(a_{47}^{*}\right)$, it is enough to show that for all $k \geqslant 0$ we have

$$
\psi_{a_{47}^{*}}^{(k)}\left(-B\left(a_{47}^{*}\right)\right) \geqslant 0 .
$$

Again, the most convenient form to use is the partial fraction decomposition of $\psi_{a_{47}^{*}}(x)$ (this decomposition also reveals that $\psi_{a_{47}^{*}}$ has no poles in the interval $\left[-B\left(a_{47}^{*}\right), 0\right]$, hence Theorem 1.14 is applicable). However, in this case, due to the high degree polynomial involved in the definition of $a_{47}^{*}$, Mathematica could not find the exact partial fraction decomposition of $\psi_{a_{47}^{*}}(x)$ in a reasonable amount of time (that is, expressing the poles and the coefficients 


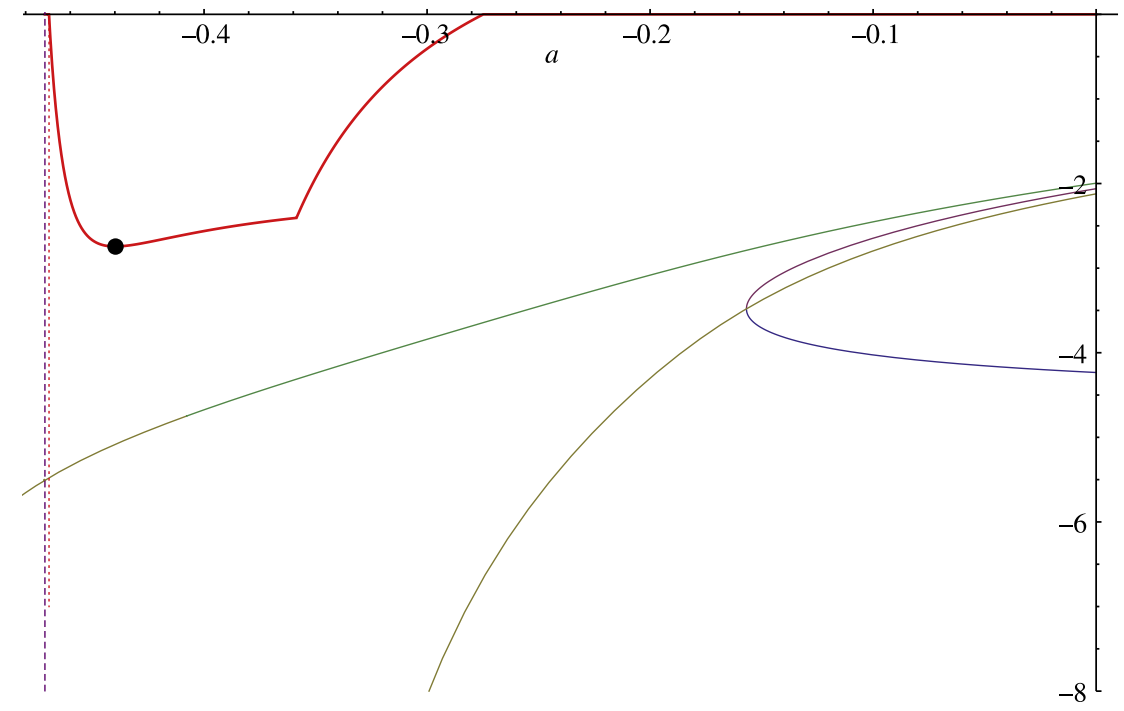

Figure 5. The graph of the function $a \mapsto-B(a)$ (red, thick curve carrying the black dot) for $\varrho_{472} \leqslant a \leqslant 0$ is depicted together with the roots of the 0 th and 1st derivatives of $\psi_{a}(\cdot)$ in the $\Pi_{4 / 4,7}$ class. Notice that $B(a)=0$ also between the two vertical dashed lines on the left. The distance between the black dot and the horizontal axis is the optimal radius of absolute monotonicity.

of the partial fractions as explicit and exact algebraic numbers). In order to overcome this difficulty, we have employed complex interval arithmetic with rational endpoints. We started from good enough lower and upper rational bounds on $a_{47}^{*}, B\left(a_{47}^{*}\right)$ and the four poles: we applied validated numerical algorithms (such as IsolatingInterval, with tolerance $10^{-20}$ ) in the case of real algebraic numbers, and higher precision evaluation in the case of complex roots, which have been previously shown to be the unique roots within larger rational rectangles in the complex plane by IsolatingInterval. Then we also expressed the coefficients of the partial fraction decomposition in terms of the poles and $a_{47}^{*}$ symbolically in advance. At the end, we were able to give rigorous lower and upper rational bounds on the (absolute value of the) coefficients using only rational arithmetic without any difficulty. These computations produced quite lengthy outputs, since the numerators and denominators of some intermediate rational numbers in the bounding intervals consisted of integers with more than 220 digits. Nevertheless, with the above simple interval technique we were able to completely reproduce the numerical partial fraction decomposition of $\psi_{a_{47}^{*}}(x)$ obtained directly in a much simpler manner.

The partial fraction decomposition of $\psi_{a_{47}^{*}}(x)$ has the form

$$
c+\frac{c_{0}}{\alpha_{0}-x}+\frac{c_{1}}{\alpha_{1}-x}+\frac{c_{2}}{\alpha_{2}-x}+\frac{\overline{c_{2}}}{\overline{\alpha_{2}}-x}
$$

with some $c, c_{0}, c_{1} \in \mathbb{R}, \alpha_{0}, \alpha_{1}>0$ and $c_{2}, \alpha_{2} \in \mathbb{C} \backslash \mathbb{R}$, further, the following numerical approximations are valid (to simplify our presentation, we now omit listing any exact rational bounds):

$$
\begin{gathered}
c \approx 11.666934, \quad c_{0} \approx 202.617318, \quad \alpha_{0} \approx 5.779490, \\
c_{1} \approx-1084.668490, \quad \alpha_{1} \approx 47.331517, \\
c_{2} \approx-19.880058-i \cdot 86.974803, \quad \alpha_{2} \approx 4.778363+i \cdot 4.007962 .
\end{gathered}
$$


For $k \geqslant 1, \psi_{a_{47}^{*}}^{(k)}(x)$ can be written as

$$
k !\left(\alpha_{0}-x\right)^{-k-1}\left(c_{0}+c_{1}\left(\frac{\alpha_{0}-x}{\alpha_{1}-x}\right)^{k+1}+c_{2}\left(\frac{\alpha_{0}-x}{\alpha_{2}-x}\right)^{k+1}+\overline{c_{2}}\left(\frac{\alpha_{0}-x}{\overline{\alpha_{2}}-x}\right)^{k+1}\right) .
$$

Now we evaluate the above expression at $x=x^{*}:=-B\left(a_{47}^{*}\right)<0$. We see by construction that $\left|\left(\alpha_{0}-x^{*}\right) /\left(\alpha_{2}-x^{*}\right)\right|=\left|\left(\alpha_{0}-x^{*}\right) /\left(\overline{\alpha_{2}}-x^{*}\right)\right|=1$, and it can also be proved that $0<$ $\left(\alpha_{0}-x^{*}\right) /\left(\alpha_{1}-x^{*}\right)<1 / 5$. So by taking into account $\alpha_{0}>0,-x^{*}>0$ and $c_{0}>0$, a sufficient condition for the positivity of $\psi_{a_{47}^{*}}^{(k)}\left(x^{*}\right)(k \geqslant 1)$ is that $\left|c_{1}\right| \cdot(1 / 5)^{k+1}+2\left|c_{2}\right| \cdot 1<c_{0}$, or, by using the rigorous bounds $\left|c_{1}\right|<1085,\left|c_{2}\right|<90$ and $c_{0}>202$, a weaker sufficient condition is given by $1085 \cdot(1 / 5)^{k+1}+180<202$, which is seen to hold for $k \geqslant 2$. But for $k=0$ and $k=1$ one checks directly (with simple rational bounds on $a_{47}^{*}$ and $-B\left(a_{47}^{*}\right)$, and even without using partial fraction decomposition) that $\psi_{a_{47}^{*}}^{(k)}\left(-B\left(a_{47}^{*}\right)\right)>0$.

\section{Determination of $\widehat{R}_{3 / 3, p}$ for $2 \leqslant p \leqslant 4$}

\subsection{Determination of $\widehat{R}_{3 / 3,4}$}

TheOREM 6.1. We have $\widehat{R}_{3 / 3,4}=\left|x^{*}\right| \approx 3.287278$ with $x^{*}$ given by $(6.2)$, and if $\psi \in \widehat{\Pi}_{3 / 3,4}$ with $R(\psi)=\widehat{R}_{3 / 3,4}$, then $\psi$ is of the form (6.1) with

$$
\begin{gathered}
a=\operatorname{root}_{1}(24,-36,12,-1) \approx 0.128886, \quad a_{1}=\operatorname{root}_{3}(8,12,-12,1) \approx 0.613341, \\
a_{2}=\operatorname{root}_{2}(64,-48,0,1) \approx 0.163176, \quad a_{3}=\operatorname{root}_{2}(1536,-1152,-24,1) \approx 0.021031 .
\end{gathered}
$$

Proof. Any element of $\widehat{\Pi}_{3 / 3,4}$ can be represented in the form

$$
\psi(z)=\frac{a_{3} z^{3}+a_{2} z^{2}+a_{1} z+1}{(1-a z)^{3}}
$$

with suitable real parameters $a, a_{1}, a_{2}$ and $a_{3}$. One directly computes that there are exactly three solutions $\left(a, a_{1}, a_{2}, a_{3}\right) \in \mathbb{R}^{4}$ to the system

$$
\psi^{(k)}(0)=1, \quad k=0,1,2,3,4
$$

(we note that we would get the same three real solutions if we allowed $\left.\left(a, a_{1}, a_{2}, a_{3}\right) \in \mathbb{C}^{4}\right)$. For all of these three solutions, condition $a>0$ is automatically satisfied (cf. Theorem 1.12), and we have for $m=1,2,3$ that $a=\operatorname{root}_{m}(24,-36,12,-1), a_{1}=\operatorname{root}_{4-m}(8,12,-12,1)$, $a_{2}=\operatorname{root}_{\frac{1}{2}\left(3 m^{2}-11 m+12\right)}(64,-48,0,1)$ and $a_{3}=\operatorname{root}_{\frac{1}{2}\left(3 m^{2}-11 m+12\right)}(1536,-1152,-24,1)$. Let us denote the corresponding rational functions by $\psi_{34 m}(m=1,2,3)$. We can check that $1 / a$ is a pole of order 3 in each case, so no cancellation between the numerator and denominator occurs. We are going to prove that

$$
\max _{m=1,2,3} R\left(\psi_{34 m}\right)=-x^{*}
$$

with

$$
\begin{aligned}
x^{*} & :=\operatorname{root}_{1}(1,12,60,120,-144,-1152,-1536,1152,2304,-1536) \\
& \approx-3.287278451851993925371346
\end{aligned}
$$

and the maximum occurring at $m=1$. 
Let us consider the $m=2$ case first. Then $\psi_{342}^{(6)}(0)=\operatorname{root}_{1}(8,-3540,600,4625)<0$, so $R\left(\psi_{342}\right)=0$ by definition.

Next for $m=3, \psi_{343}^{(5)}\left(\operatorname{root}_{5}(1,66,1242,7008,1872,-648,-72)\right)=0$, so Theorem 1.13 with $\ell=5$ says that $R\left(\psi_{343}\right) \leqslant-\operatorname{root}_{5}(1,66,1242,7008,1872,-648,-72) \approx 0.0943315<\left|x^{*}\right|$.

Finally, for $m=1$ the partial fraction decomposition of $\psi_{341}$ reads as

$$
\psi_{341}(x)=c+\sum_{\ell=1}^{3} \frac{c_{\ell}}{\left(\alpha_{0}-x\right)^{\ell}},
$$

where $c=\operatorname{root}_{1}(1,9,-9,-9) \approx-9.82294, c_{1}=\operatorname{root}_{3}(1,-288,-5184,13824) \approx 304.856$, $c_{2}=\operatorname{root}_{1}(1,3168,-243648,-235008) \approx-3243.10, c_{3}=\operatorname{root}_{3}(1,-11808,-684288,110592) \approx$ 11865.6 , and $\alpha_{0}=\operatorname{root}_{3}(1,-12,36,-24) \approx 7.75877$. Then $\psi_{341}\left(x^{*}\right)=0$, so by Theorem 1.13 with $\ell=0$ we have $R\left(\psi_{343}\right) \leqslant\left|x^{*}\right|$. On the other hand, since now trivially $B\left(\psi_{341}\right)=+\infty$, Theorem 1.14 with $x:=x^{*}$ proves absolute monotonicity of $\psi_{341}$ on $\left[x^{*}, 0\right]$ provided that we show the point conditions

$$
\psi_{341}^{(k)}\left(x^{*}\right) \geqslant 0
$$

for all $k \geqslant 1$ (the theorem is applicable due to the lack of poles in $(-\infty, 0])$. Indeed, for such $k$ values we have

$$
\psi_{341}^{(k)}\left(x^{*}\right)=(k+2) !\left(\alpha_{0}-x^{*}\right)^{-k-3}\left[\frac{c_{3}}{2}+\frac{c_{2}}{k+2}\left(\alpha_{0}-x^{*}\right)+\frac{c_{1}}{(k+1)(k+2)}\left(\alpha_{0}-x^{*}\right)^{2}\right] .
$$

Clearly, because of $\alpha_{0}-x^{*}>0$, it is enough to verify that the [...] expression is non-negative, but since $\lim _{k \rightarrow+\infty}[\ldots]=c_{3} / 2>0$, only finitely many $k$ values should be checked. We easily see that $[\ldots] \geqslant 0$ holds if, for example,

$$
\frac{\alpha_{0}-x^{*}}{k+2}\left(\left|c_{2}\right|+\frac{c_{1}}{k+1}\left(\alpha_{0}-x^{*}\right)\right)<\frac{23 / 2}{k+2}\left(3250+\frac{305}{k+1} \cdot \frac{23}{2}\right)<5500<\frac{c_{3}}{2} .
$$

Now the second inequality above is satisfied for $k \geqslant 6$, and we determine directly that [...]>0 holds for each $k=1,2, \ldots, 5$ as well, completing the argument that the maximal radius of absolute monotonicity within the $\widehat{\Pi}_{3 / 3,4}$ class is $\left|x^{*}\right|$.

Remark 6.2. Partial fraction decomposition in the $m=2$ case shows that the corresponding 'dominant coefficient' $c_{3}$ is negative, immediately implying $R\left(\psi_{342}\right)=0$ (cf. Remark 8.12).

\subsection{Determination of $\widehat{R}_{3 / 3,3}$}

TheOrEm 6.3. We have $\widehat{R}_{3 / 3,3}=2+\sqrt{8} \approx 4.82842$, and if $\psi \in \widehat{\Pi}_{3 / 3,3}$ with $R(\psi)=\widehat{R}_{3 / 3,3}$, then $\psi$ is determined by (6.3) with $a=a_{33}^{*}:=(2-\sqrt{2}) / 4$.

Proof. Now the one-parameter family of rational functions takes the form

$$
\psi_{a}(z)=\frac{\frac{1}{6}\left(-6 a^{3}+18 a^{2}-9 a+1\right) z^{3}+\frac{1}{2}\left(6 a^{2}-6 a+1\right) z^{2}+(1-3 a) z+1}{(1-a z)^{3}}
$$

with $a \in \mathbb{R}$. For $a=0$ the rational function reduces to the third degree Taylor polynomial of the exponential function around 0 , which has $R=1$. So we can assume $a \neq 0$. Let us exclude two more exceptional parameter values as well. It is easily seen by computing the corresponding resultant that the numerator and denominator have a common root if and only if $a=\frac{1}{6}(3 \pm \sqrt{3})$. In any of these cases, $\psi_{a}$ is either $\psi_{231}$ or $\psi_{232}$ from the $\widehat{\Pi}_{2 / 2,3}$ class (see (2.1) $-(2.2))$, hence $R \leqslant 1+\sqrt{3}<2+\sqrt{8}$. 


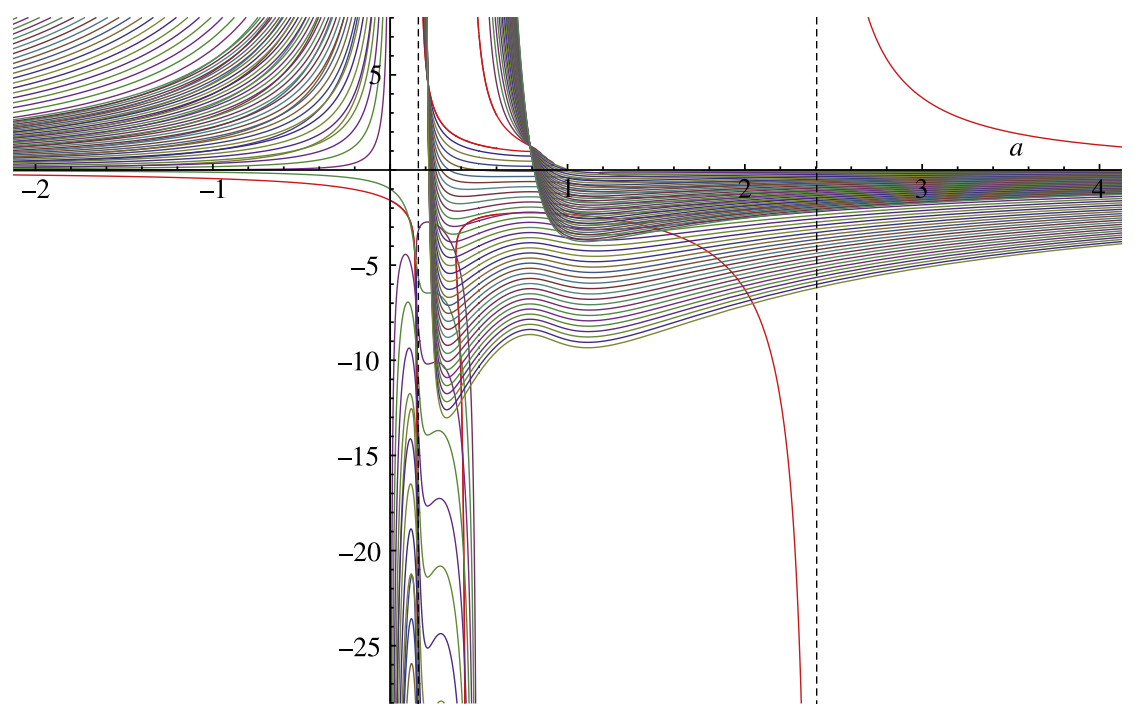

FIGURE 6. Each curve (corresponding to different $k$ values between $0,1, \ldots, 36$ ) shows a root of $\psi_{a}^{(k)}(\cdot)$ as the parameter $a$ is varied in the $\widehat{\Pi}_{3 / 3,3}$ class. The red curve $(k=0)$ is obtained as a solution of a parametric cubic polynomial, whereas the other curves are derived from quadratic ones.

Now we can apply Theorem 1.12 saying that $a>0$ is necessary to have $R>0$. So in the following we can suppose that $0<a \neq \frac{1}{6}(3 \pm \sqrt{3})$.

One can prove by induction that for $k \geqslant 1$

$$
\begin{aligned}
\psi_{a}^{(k)}(x)= & \frac{a^{k-3} k !}{2}(1-a x)^{-k-3} \\
& \quad \times\left((2 a-1)(6 a-1) a^{2} x^{2}+\left(8 a^{3}(k-2)+a^{2}(9-7 k)+a(k-1)\right) x\right. \\
& \left.\quad+\left(a^{2}(k-3)(k-2)-a(k-3)(k-1)+\frac{1}{6}(k-2)(k-1)\right)\right) .
\end{aligned}
$$

Let us consider first the $k=5$ special case of the above explicit formula with $a \geqslant \frac{1}{3}$. From this we see that one of the roots of $\psi_{a}^{(5)}(\cdot)$ is $-\frac{1}{3}$ for $a=\frac{1}{2}$, and

$$
\frac{-12 a^{2}+13 a-2-\sqrt{72 a^{4}-168 a^{3}+123 a^{2}-28 a+2}}{a(2 a-1)(6 a-1)}
$$

for $\frac{1}{3} \leqslant a \neq \frac{1}{2}$. But this last expression has values in, say, $(-1,0]$ for $\frac{1}{3} \leqslant a \neq \frac{1}{2}$, implying, by Theorem 1.13 with $\ell=5$, that $R\left(\psi_{a}\right) \leqslant 1$ for $\frac{1}{3} \leqslant a$.

We focus on the remaining $a \in\left(0, \frac{1}{3}\right) \backslash\{(3-\sqrt{3}) / 6\}$ parameter set now. For $0<a<$ $(2-\sqrt{2}) / 4, \psi_{a}^{\prime \prime}(\cdot)$ has a root of the form

$$
\frac{5 a-1+\sqrt{-48 a^{3}+57 a^{2}-14 a+1}}{2 a(2 a-1)(6 a-1)}
$$

in the interval $(-2-\sqrt{8},-1)$, meaning (see Theorem 1.13) that $R\left(\psi_{a}\right)<2+\sqrt{8}$ here. On the other hand, for $(2-\sqrt{2}) / 4<a<\frac{1}{3}$ (the exceptional $(3-\sqrt{3}) / 6$ value can safely be added again), $\psi_{a}^{\prime}(\cdot)$ has a root of the form

$$
\frac{4 a-1+\sqrt{-8 a^{2}+8 a-1}}{(2 a-1)(6 a-1)}
$$




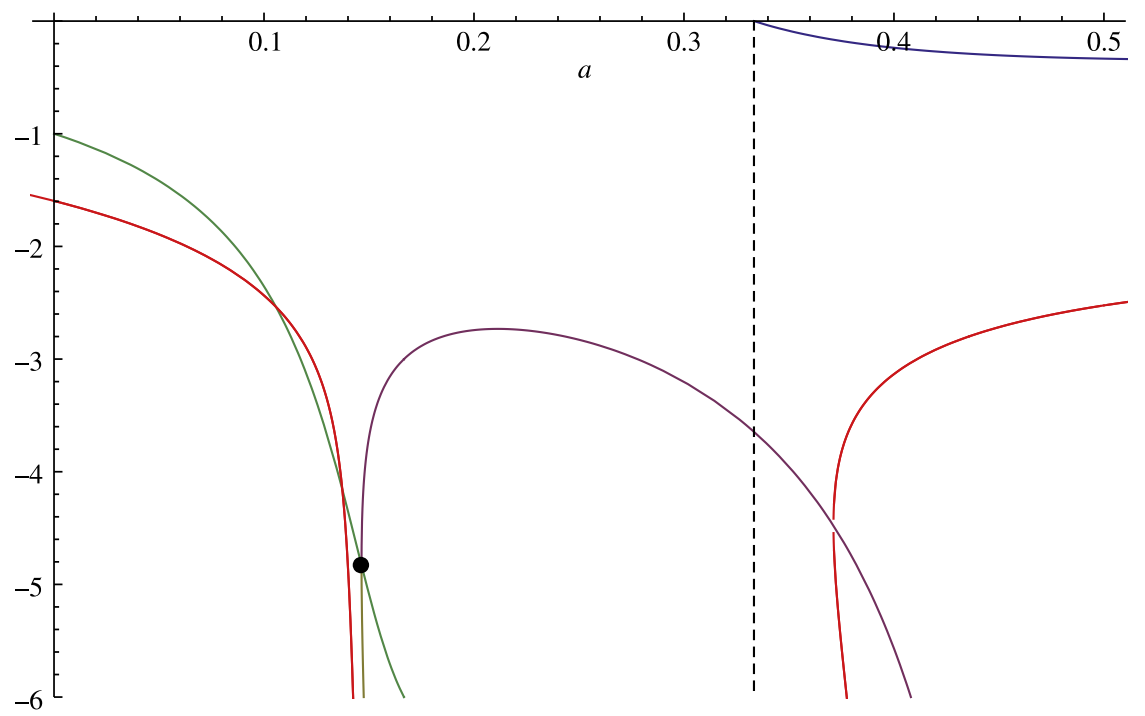

FIGURE 7. Zooming in on Figure 6 (with some curves omitted) reveals the location of the optimum (depicted as a black dot) in the $\widehat{\Pi}_{3 / 3,3}$ class.

in the interval $\left(-2-\sqrt{8},-1-\sqrt{7}\right.$ ), so (again by Theorem 1.13) $R\left(\psi_{a}\right)<2+\sqrt{8}$, too, for these $a$ values. (The $a=\frac{1}{6}$ case is a removable singularity of the above root expression with value -3 .)

Finally, for $a=a_{33}^{*}:=(2-\sqrt{2}) / 4, \psi_{a_{33}^{*}}(-2-\sqrt{8})=1-2 \sqrt{2} / 3>0$, and, by using $(6.4)$, for any $k \geqslant 1$ we have

$$
\psi_{a_{33}^{*}}^{(k)}(-2-\sqrt{8})=\frac{4}{3}\left(\frac{3}{2}-\sqrt{2}\right)^{k} k !(k-1)(k-2) \geqslant 0,
$$

showing that $\psi_{a_{33}^{*}}$ is absolutely monotonic at $x=-2-\sqrt{8}$. But then Theorem 1.14 (applicable because there are no poles in $(-\infty, 0])$ guarantees absolute monotonicity on the whole interval $[-2-\sqrt{8}, 0]$, and Theorem 1.13 with, say, $\ell=1$ shows that $R\left(\psi_{a_{33}^{*}}\right) \leqslant 2+\sqrt{8}$, completing the proof.

\subsection{Determination of $\widehat{R}_{3 / 3,2}$}

In this section we finish the proof of Theorem 2.2 in the $s=3$ case.

As we have seen in $\S 2.4 .2,(2.5)$ and Lemma 2.7 imply that now we can restrict our attention to rational functions of the form

$$
\psi_{a, c}(z)=\frac{c z^{3}+\left(\frac{1}{2}-3 a+3 a^{2}\right) z^{2}+(1-3 a) z+1}{(1-a z)^{3}}
$$

with suitable parameters $a>0$ and $c \in \mathbb{R}$. For simplicity, we have used and will use the letter $c$ instead of $a_{3}$. We are going to show that

$$
\psi_{a, c}(-6) \geqslant 0, \quad \psi_{a, c}^{\prime}(-6) \geqslant 0, \quad \psi_{a, c}^{\prime \prime}(-6) \geqslant 0
$$

and

$$
\forall k \in \mathbb{N}, k \geqslant 3: \quad \psi_{a, c}^{(k)}(-6) \geqslant 0
$$

imply that $a=\frac{1}{6}$ and $c=\frac{1}{216}$, see Figure 8 . 


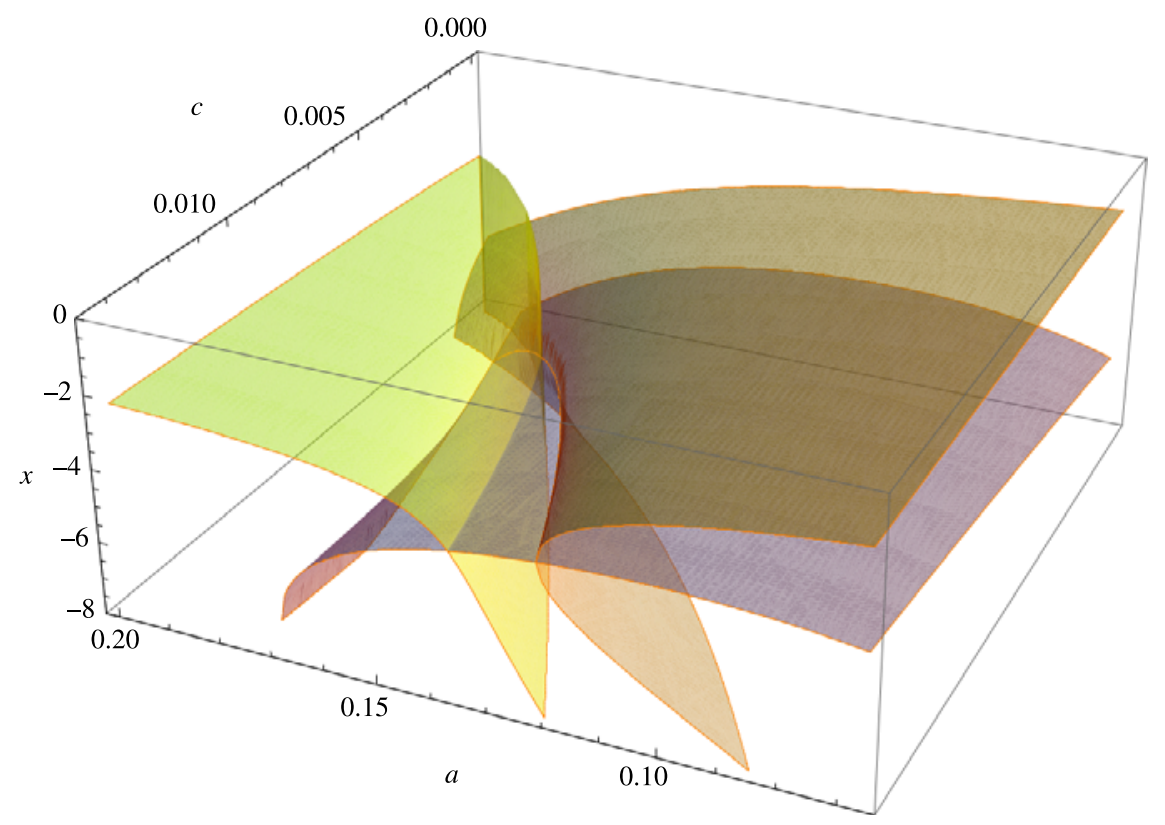

FiguRE 8. The $\widehat{\Pi}_{3 / 3,2}$ class. Depicting the roots of $\psi_{a, c}^{(k)}(\cdot)$ for $k=0,1,2$ as a function of $(a, c)$, the optimal point is obtained as the intersection of these three surfaces at $(a, c, x)=\left(\frac{1}{6}, \frac{1}{216},-6\right)$.

First, by using (2.7) evaluated at $x=-6$, we see that these inequalities are equivalent to

$$
\begin{gathered}
108 a^{2}-90 a-216 c+13 \geqslant 0, \\
108 a^{3}-108 a^{2}+42 a+108 c-5 \geqslant 0, \\
108 a^{4}-126 a^{3}+72 a^{2}-12 a+1 / 2+(108 a-18) c \geqslant 0, \\
k^{2}\left(a^{3}-2 a^{2}+a / 2+c\right)+k\left(-36 a^{4}+57 a^{3}-8 a^{2}-36 a c-a / 2-3 c\right) \\
+36 a c+2 c+216 a^{5}-180 a^{4}+26 a^{3}+216 a^{2} c \geqslant 0,
\end{gathered}
$$

where, of course, (6.8) should hold for all $k \geqslant 3$ integers. We will also use the necessary condition (2.6), which now reads as

$$
a^{3}-2 a^{2}+a / 2+c \geqslant 0
$$

(being just the leading $k$-coefficient of (6.8)).

Let us express the linear parameter, $c$ from $(6.5),(6.6)$ and (6.9), then combine the resulting inequalities to eliminate $c$ and get

$$
\frac{-108 a^{3}+108 a^{2}-42 a+5}{108} \leqslant \frac{108 a^{2}-90 a+13}{216} \geqslant \frac{-2 a^{3}+4 a^{2}-a}{2} .
$$

Rearranging both inequalities to 0 , the two cubic polynomials can be nicely factorized (containing factors $2 a-1,6 a-1,6 a+1$ and $36 a^{2}-60 a+13$ ), so this, together with $a>0$, implies that

$$
0<a \leqslant \frac{1}{6} \quad \text { or } a \geqslant \frac{5+2 \sqrt{3}}{6} .
$$

Now let us consider (6.7) and separate two cases according to the coefficient of $c$.

If it vanishes, that is, for $a=\frac{1}{6}$, then (6.5) and (6.6) yield $c=\frac{1}{216}$, and we are ready. 
Hence the proof is finished as soon as we have shown that (6.10) and $a \neq \frac{1}{6}$ lead to a contradiction.

First suppose that $0<a<\frac{1}{6}$. Then an elementary calculation shows that (6.7) (whose left-hand side is just $\left.(6 a-1)\left(36 a^{3}-36 a^{2}+18 a-1+36 c\right) / 2\right)$ and $(6.6)$ contradict each other.

Consequently, it is sufficient to exclude $a \geqslant(5+2 \sqrt{3}) / 6$. Let us express $c$ (again) from (6.9) and (6.5) to have

$$
\frac{-2 a^{3}+4 a^{2}-a}{2} \leqslant c \leqslant \frac{108 a^{2}-90 a+13}{216}
$$

and let us abbreviate the left-hand side of $(6.8)$ by $\lambda(k, a, c)$. The lemma below completes the proof.

Lemma 6.4. For each $a \geqslant(5+2 \sqrt{3}) / 6$ and each $c$ satisfying (6.11), there is an integer $k \geqslant 3$ such that $\lambda(k, a, c)<0$.

Proof. Let us pick and fix throughout the proof an arbitrary $a \geqslant(5+2 \sqrt{3}) / 6$ and $c$ satisfying (6.11). Since $\partial_{c} \lambda(k, a, c)=k^{2}-(36 a+3) k+216 a^{2}+36 a+2$, we see that $\partial_{c} \lambda(k, a, c)<0$ holds if and only if

$$
\varrho_{321}^{-}(a)<k<\varrho_{321}^{+}(a),
$$

where $\varrho_{321}^{ \pm}(a):=\frac{3}{2}(12 a+1) \pm \frac{1}{2} \sqrt{432 a^{2}+72 a+1}$. It is also easily seen that, for the allowed $a$ values, (6.12) automatically implies $k \geqslant 3$. So, if $k$ satisfies (6.12), then due to (6.11) we have that

$$
\lambda(k, a, c) \leqslant \lambda\left(k, a, \frac{-2 a^{3}+4 a^{2}-a}{2}\right)=a(6 a+1)\left((1-2 a) k+36 a^{2}-8 a-1\right) .
$$

Since $a>\frac{1}{2}$ now, this very last factor is negative if and only if

$$
k>\frac{36 a^{2}-8 a-1}{2 a-1} .
$$

These mean that (6.12) and (6.13) imply $\lambda(k, a, c)<0$. But $\varrho_{321}^{-}(a)<\left(36 a^{2}-8 a-1\right) /(2 a-1)$ holds precisely if $a \in\left(0, \frac{1}{6}\right) \cup\left(\frac{1}{2},+\infty\right)$, which is now true by the assumption on $a$, so to finish the proof, it is enough to show that the set

$$
\left(\frac{36 a^{2}-8 a-1}{2 a-1}, \varrho_{321}^{+}(a)\right) \cap \mathbb{N}
$$

is not empty: an elementary computation yields that for $a \geqslant(5+2 \sqrt{3}) / 6$ we have

$$
\varrho_{321}^{+}(a)-\frac{36 a^{2}-8 a-1}{2 a-1}>9 .
$$

REMARK 6.5. We add that this last integer in the proof, 9, could not be replaced by, say, 10 on the whole interval $a \in((5+2 \sqrt{3}) / 6,+\infty)$.

REMARK 6.6. It is possible to finish the proof of Lemma 6.4 by using the $\partial_{c} \lambda(k, a, c)>0$ case, but this would result in more difficult computations and would provide (asymptotically) an interval of length 6 (instead of 9) in the last step.

\section{Determination of $\widehat{R}_{4 / 4, p}$ for $2 \leqslant p \leqslant 5$}

For the sake of brevity and due to the fact that the techniques used in $\S \S 7.1-7.3$ are very similar to the corresponding earlier ones, we provide only brief (indication of the) proofs there. 
However, the complete proof in $\S 7.4$ is presented, because that result, similarly to the one in $\S 6.3$, is a special case of Conjecture 1.6.

\subsection{Determination of $\widehat{R}_{4 / 4,5}$}

TheOrem 7.1. We have $\widehat{R}_{4 / 4,5}=\left|x^{*}\right| \approx 3.743299$ with $x^{*}$ given by $(7.3)$, and if $\psi \in \widehat{\Pi}_{4 / 4,5}$ with $R(\psi)=\widehat{R}_{4 / 4,5}$, then $\psi$ is of the form (7.1) with $a_{4}, a_{3}, a_{2}, a_{1}, a$ defined by $(7.2)$ and $m_{4}=m_{3}=m_{2}=3, m_{1}=4, m_{0}=1$.

Proof. The proof is completely analogous to the one in $\S 6.1$. There are exactly four rational functions in this $\widehat{\Pi}_{4 / 4,5}$ class:

$$
\psi(z)=\frac{a_{4} z^{4}+a_{3} z^{3}+a_{2} z^{2}+a_{1} z+1}{(1-a z)^{4}}
$$

where

$$
\begin{gathered}
a_{4}=\operatorname{root}_{m_{4}}(2160000,4032000,-93600,-120,1), \quad a_{3}=\operatorname{root}_{m_{3}}(18000,24000,-1800,0,1), \\
a_{2}=\operatorname{root}_{m_{2}}(1200,-7200,120,200,3), \quad a_{1}=\operatorname{root}_{m_{1}}(15,60,-30,-20,7), \\
a=\operatorname{root}_{m_{0}}(120,-240,120,-20,1),
\end{gathered}
$$

with

$$
\left(m_{4}, m_{3}, m_{2}, m_{1}, m_{0}\right) \in\{(1,1,4,1,4),(2,2,2,3,2),(3,3,3,4,1),(4,4,1,2,3)\} .
$$

First we note that in all four cases the only pole of $\psi$ is positive real.

For $m_{4}=1$ the corresponding $\psi$ satisfies $\psi^{(5)}(-1 / 2)<0$, hence $R(\psi)<1 / 2$.

For $m_{4}=2$ the corresponding $\psi$ satisfies $\psi^{(8)}(0)<0$, hence $R(\psi)=0$.

For $m_{4}=4$ the corresponding $\psi$ satisfies $\psi^{(7)}(-1 / 2)<0$, hence $R(\psi)<1 / 2$.

For $m_{4}=3$ the corresponding $\psi$ satisfies $\psi^{\prime}\left(x^{*}\right)=0$ with

$$
\begin{aligned}
x^{*} & :=\operatorname{root}_{1}(1,12,48,-32,-864,-2016,2784,13248,-9072,-35136,44928,-20736,3456) \\
& \approx-3.743299247417768882803493,
\end{aligned}
$$

hence by Theorem 1.13 with $\ell=1$, it has $R(\psi) \leqslant\left|x^{*}\right|$. By using partial fraction decomposition, we can show that $R(\psi)=\left|x^{*}\right|$, therefore $\widehat{R}_{4 / 4,5}=\left|x^{*}\right|$.

\subsection{Determination of $\widehat{R}_{4 / 4,4}$}

THEOREM 7.2. We have $\widehat{R}_{4 / 4,4} \approx 5.167265$ (given exactly by (7.5)), and if $\psi \in \widehat{\Pi}_{4 / 4,4}$ with $R(\psi)=\widehat{R}_{4 / 4,4}$, then $\psi$ is determined by (7.4) and (7.6).

Proof. We refer to $\S 6.2$ for an analogous treatment and for further details on the theorems used. Elements of this class can be written as

$$
\begin{aligned}
\psi_{a}(z)=(1-a z)^{-4} & \left(\left(a^{4}-4 a^{3}+3 a^{2}-\frac{2 a}{3}+\frac{1}{24}\right) z^{4}+\left(-4 a^{3}+6 a^{2}-2 a+\frac{1}{6}\right) z^{3}\right. \\
+ & \left.\left(6 a^{2}-4 a+\frac{1}{2}\right) z^{2}+(1-4 a) z+1\right)
\end{aligned}
$$


with a real parameter $a$. Here also we can assume that $a>0$ and $\psi_{a}$ does not have removable singularities (indeed, $a=0$ implies $R=1 ; a<0$ without removable singularities implies $R=0$; while if the numerator and denominator have a common root, then $\psi_{a}$ is one of the three rational functions in $\S 6.1$, hence $R \leqslant \widehat{R}_{3 / 3,4}<5$ ). By applying the same techniques as earlier in the one-parameter families, we can prove that

$$
\begin{aligned}
\widehat{R}_{4 / 4,4} & =\operatorname{root}_{3}(1,-24,240,-1168,1848,7008,-30528,7488,71568,36864) \\
& \approx 5.167265421277419938673374,
\end{aligned}
$$

with the unique optimal rational function corresponding to the parameter value

$$
\begin{aligned}
a=a_{44}^{*}:= & \operatorname{root}_{1}(147456,-546624,799488,-601344,258432, \\
& -66576,10400,-960,48,-1) \\
\approx & 0.09713312764144710280835106 .
\end{aligned}
$$

\subsection{Determination of $\widehat{R}_{4 / 4,3}$}

This class can be described by two real parameters. We can prove along the lines of the uniqueness-type proof in $\S 6.3$ that

$$
\widehat{R}_{4 / 4,3}=3+\sqrt{15} \approx 6.872983
$$

and the unique optimal rational function in this class is given by

$$
\psi(z):=\frac{\frac{1}{900}(52 \sqrt{15}-201) z^{4}+\left(\frac{1}{6}-\frac{1}{5} \sqrt{\frac{3}{5}}\right) z^{3}+\left(\frac{9}{10}-\sqrt{\frac{3}{5}}\right) z^{2}+\left(2 \sqrt{\frac{3}{5}}-1\right) z+1}{\left(1-\frac{1}{10}(5-\sqrt{15}) z\right)^{4}} .
$$

Interestingly, the first derivative of $\psi$ has a simple structure, see Table 2.3.

\subsection{Determination of $\widehat{R}_{4 / 4,2}$}

In this section we finish the proof of Theorem 2.2 in the $s=4$ case. We will apply the same principles as in $\S 6.3$, but, this time, we could eliminate the 'linear' parameters $d$ and $c$, recursively via monotonicity, only at the cost of a little bit more computation.

By appealing to formula (2.5) and Lemma 2.7 again, it is sufficient to consider rational functions of the form

$$
\psi_{a, c, d}(z)=\frac{d z^{4}+c z^{3}+\left(\frac{1}{2}-4 a+6 a^{2}\right) z^{2}+(1-4 a) z+1}{(1-a z)^{4}}
$$

with suitable parameters $a>0$ and $c, d \in \mathbb{R}$. (Again, for easier readability we have used and will use $c$ instead of $a_{3}$, and $d$ instead of $a_{4}$.) We show that

$$
\psi_{a, c, d}(-8) \geqslant 0, \quad \psi_{a, c, d}^{\prime}(-8) \geqslant 0, \quad \psi_{a, c, d}^{\prime \prime}(-8) \geqslant 0, \quad \psi_{a, c, d}^{\prime \prime \prime}(-8) \geqslant 0
$$

and

$$
\forall k \in \mathbb{N}, k \geqslant 4: \quad \psi_{a, c, d}^{(k)}(-8) \geqslant 0
$$

imply $a=\frac{1}{8}, c=\frac{1}{128}$ and $d=\frac{1}{4096}$. 
Let us use (2.7) at $x=-8$ (dropping the always positive factors again) and write out the above inequalities in detail to get

$$
\begin{aligned}
& 1-8(1-4 a)+64\left(6 a^{2}-4 a+\frac{1}{2}\right)-512 c+4096 d \geqslant 0, \\
& (8 a+1)\left(1-4 a-16\left(6 a^{2}-4 a+\frac{1}{2}\right)+192 c-2048 d\right) \\
& \quad+4 a\left(1-8(1-4 a)+64\left(6 a^{2}-4 a+\frac{1}{2}\right)-512 c+4096 d\right) \geqslant 0, \\
& 10 a^{2}\left(1-8(1-4 a)+64\left(6 a^{2}-4 a+\frac{1}{2}\right)-512 c+4096 d\right) \\
& \quad+4 a(8 a+1)\left(1-4 a-16\left(6 a^{2}-4 a+\frac{1}{2}\right)+192 c-2048 d\right) \\
& \quad+\frac{1}{2}(8 a+1)^{2}\left(2\left(6 a^{2}-4 a+\frac{1}{2}\right)-48 c+768 d\right) \geqslant 0, \\
& 10 a^{2}(8 a+1)\left(1-4 a-16\left(6 a^{2}-4 a+\frac{1}{2}\right)+192 c-2048 d\right) \\
& \quad+2 a(8 a+1)^{2}\left(2\left(6 a^{2}-4 a+\frac{1}{2}\right)-48 c+768 d\right)+\frac{1}{6}(8 a+1)^{3}(6 c-192 d) \\
& \quad+20 a^{3}\left(1-8(1-4 a)+64\left(6 a^{2}-4 a+\frac{1}{2}\right)-512 c+4096 d\right) \geqslant 0, \\
& k^{3}\left(6 a^{4}-6 a^{3}+a^{2}+2 a c+2 d\right) \\
& \quad+k^{2}\left(-384 a^{5}+324 a^{4}-42 a^{3}-144 a^{2} c-6 a c-192 a d-12 d\right) \\
& \quad+k\left(4608 a^{6}-3072 a^{5}+618 a^{4}+2304 a^{3} c-36 a^{3}+144 a^{2} c+4608 a^{2} d\right. \\
& \left.\quad-a^{2}+4 a c+576 a d+22 d\right) \\
& \quad+4608 a^{6}-2688 a^{5}-6144 a^{4} c+300 a^{4}-24576 a^{3} d-4608 a^{2} d-384 a d-12 d \geqslant 0,
\end{aligned}
$$

with (7.11) valid for any integer $k \geqslant 4$. The necessary condition (2.6) now reads as

$$
6 a^{4}-6 a^{3}+a^{2}+2 a c+2 d \geqslant 0 .
$$

The domain of the 'non-linear' parameter $a>0$ will be divided at the following 'natural' points (determined by the requirement that denominators in the proof below have constant sign on the corresponding intervals)

$$
0<\frac{3-\sqrt{5}}{16}<\frac{1}{12}<\frac{1}{8}<\frac{3}{16}<\frac{3+\sqrt{5}}{16}<\frac{9+2 \sqrt{6}}{24}<\ldots
$$

and also at the 'artificial' point $\ldots<\frac{22}{10}$ (introduced for technical reasons). The explicit formulae for the $P_{42 n}$ and $\varrho_{42 n}$ expressions $(n=1,2, \ldots)$ appearing soon are listed in the Appendix (or directly in the proofs).

The interval $a \in(0,(3-\sqrt{5}) / 16)$. From $(7.10), d$ is expressed as $d \leqslant P_{421}(a, c) /\left(2048 a^{2}-\right.$ $768 a+32)$, while from $(7.9)$ as $d \geqslant P_{422}(a, c) /(4096 a-768)$. By joining these inequalities, $d$ is eliminated and we get

$$
c \leqslant \frac{-6144 a^{5}+5120 a^{4}-2144 a^{3}+400 a^{2}-32 a+1}{1536 a^{2}-256 a+24} .
$$

From (7.8) we obtain $d \leqslant P_{423}(a, c) / 2048$, which, together with $d \geqslant P_{422}(a, c) /(4096 a-768)$ allows us to eliminate $d$ again and get

$$
c \geqslant \frac{1}{192}\left(-768 a^{3}+512 a^{2}-144 a+13\right) .
$$

From $(7.13)$ and $(7.14)$ we get $(8 a-1)(8 a+1)(16 a-3) \leqslant 0$, which is impossible for $a \in$ $(0,(3-\sqrt{5}) / 16)$.

The case when $a=(3-\sqrt{5}) / 16$. From (7.10), $c$ can be expressed as $c \leqslant \frac{1}{128}(2 \sqrt{5}-3)$. But (7.14) now says $\frac{1}{384}(1+6 \sqrt{5}) \leqslant c$, a contradiction. 
The interval $a \in\left(\frac{1}{16}(3-\sqrt{5}), \frac{3}{16}\right)$ (containing the unique solution). We now have three lower estimates: $d \geqslant P_{422}(a, c) /(4096 a-768), d \geqslant P_{421}(a, c) /\left(2048 a^{2}-768 a+32\right)$, and (from (7.7)) $d \geqslant P_{424}(a, c) / 4096$. We also have the earlier upper estimate $d \leqslant P_{423}(a, c) / 2048$. By making three appropriate pairs from these, we eliminate $d$ in each case and get (7.14),

$$
c \leqslant \frac{1}{128}\left(192 a^{2}-104 a+11\right),
$$

and

$$
\begin{cases}c \leqslant \frac{-6144 a^{4}+4608 a^{3}-1600 a^{2}+200 a-7}{1536 a-128} & \text { for } \frac{1}{16}(3-\sqrt{5})<a<\frac{1}{12} \\ c \geqslant \frac{-6144 a^{4}+4608 a^{3}-1600 a^{2}+200 a-7}{1536 a-128} & \text { for } \frac{1}{12}<a<\frac{3}{16} .\end{cases}
$$

(It is easily seen that $a=1 / 12$ immediately leads to a contradiction.)

On $\frac{1}{16}(3-\sqrt{5})<a<\frac{1}{12},(7.14)$ and (7.16) imply $\frac{5}{3}(8 a-1)(8 a+1) \geqslant 0$, which is impossible.

For $\frac{1}{12}<a<\frac{3}{16},(7.14)$ and (7.16) imply

$$
\begin{cases}c \geqslant \frac{-6144 a^{4}+4608 a^{3}-1600 a^{2}+200 a-7}{1536 a-128} & \text { for } \frac{1}{12}<a \leqslant \frac{1}{8}, \\ c \geqslant \frac{1}{192}\left(-768 a^{3}+512 a^{2}-144 a+13\right) & \text { for } \frac{1}{8}<a<\frac{3}{16} .\end{cases}
$$

On $\frac{1}{8}<a<\frac{3}{16}$, (7.15) and (7.17) cannot be true simultaneously. On the other hand, for $\frac{1}{12}<a \leqslant \frac{1}{8},(7.15)$ and (7.17) yield $a=\frac{1}{8}$ and $c=\frac{1}{128}$, and with $d \geqslant P_{424}(a, c) / 4096$ and $d \leqslant P_{423}(a, c) / 2048$ we get $d=1 / 4096$.

The interval $a \in\left[\frac{3}{16}, \frac{1}{24}(9+2 \sqrt{6})\right.$. We will use here the two lower estimates $d \geqslant$ $P_{424}(a, c) / 4096, d \geqslant 1 / 2\left(-6 a^{4}+6 a^{3}-a^{2}-2 a c\right)$ (this second one derived from (7.12)) and the upper estimate $d \leqslant P_{423}(a, c) / 2048$. Again, by forming two appropriate pairs, $d$ is eliminated and we obtain two inequalities in $a$ and $c$. After a rearrangement, we have (7.15) and $c \geqslant \frac{1}{192}\left(-768 a^{3}+768 a^{2}-160 a+7\right)$, leading to a contradiction.

So far we have examined the region $0<a<\frac{1}{24}(9+2 \sqrt{6})$ and proved that the system has a solution if and only if $a=\frac{1}{8}$.

In the rest of this section, we will show that there are no solutions In the rest of this section, we will show that there are no solutions for $a \geqslant \frac{1}{24}(9+2 \sqrt{6})$.

It can be shown (in a few lines, by using only (7.7), (7.8) and (7.12), but skipping the details here) that for $a \geqslant \frac{1}{24}(9+2 \sqrt{6})$ we have

$$
\left\{\begin{array}{l}
\frac{1}{192}\left(-768 a^{3}+768 a^{2}-160 a+7\right) \leqslant c \leqslant \frac{1}{512}\left(-1536 a^{3}+1728 a^{2}-424 a+25\right), \\
\frac{1}{2}\left(-6 a^{4}+6 a^{3}-a^{2}-2 a c\right) \leqslant d \leqslant \frac{1}{2048}\left(768 a^{3}-512 a^{2}-512 a c+104 a+192 c-7\right)
\end{array}\right.
$$

or

$$
\left\{\begin{array}{l}
\frac{1}{512}\left(-1536 a^{3}+1728 a^{2}-424 a+25\right) \leqslant c \leqslant \frac{1}{128}\left(192 a^{2}-104 a+11\right), \\
\frac{1}{4096}\left(-384 a^{2}+224 a+512 c-25\right) \leqslant d \leqslant \frac{1}{2048}\left(768 a^{3}-512 a^{2}-512 a c+104 a+192 c-7\right) .
\end{array}\right.
$$

Let us introduce the abbreviation $\lambda(k, a, c, d)$ to denote the left-hand side of (7.11).

Lemma 7.3. For each $\frac{1}{24}(9+2 \sqrt{6}) \leqslant a \leqslant \frac{22}{10}$, and any $c$ and $d$ satisfying (7.18), we have that $\lambda(77, a, c, d)<0$. 
Proof. Since the polynomial $\partial_{d} \lambda(77, a, c, d)=-48 P_{425}(a)$ has a unique root $\varrho_{421} \approx 1.17854$ in the given $a$-interval, we separate two cases.

The interval $a \in\left[\frac{1}{24}(9+2 \sqrt{6}), \varrho_{421}\right)$. Here $\partial_{d} \lambda(77, a, c, d)>0$, so by $(7.18)$

$$
\begin{aligned}
\lambda(77, a, c, d) & \leqslant \lambda\left(77, a, c, \frac{1}{2048}\left(768 a^{3}-512 a^{2}-512 a c+104 a+192 c-7\right)\right) \\
& =\frac{57}{128}(8 a+1) P_{426}(a, c)
\end{aligned}
$$

from which we see that it is enough to show that $P_{426}(a, c)<0$. But for the current $a$ and $c$ values we have $\partial_{c} P_{426}(a, c)=192\left(128 a^{2}-800 a+925\right) \geqslant 0$, so by $(7.18)$

$$
\begin{aligned}
P_{426}(a, c) & \leqslant P_{426}\left(a, \frac{1}{512}\left(-1536 a^{3}+1728 a^{2}-424 a+25\right)\right) \\
& =\frac{1}{8}(8 a+1)\left(24576 a^{4}-139264 a^{3}+212288 a^{2}-126000 a+17575\right)<0 .
\end{aligned}
$$

The interval $a \in\left[\varrho_{421}, \frac{22}{10}\right]$. Here $\partial_{d} \lambda(77, a, c, d) \leqslant 0$, so by $(7.18)$

$$
\lambda(77, a, c, d) \leqslant \lambda\left(77, a, c, \frac{1}{2}\left(-6 a^{4}+6 a^{3}-a^{2}-2 a c\right)\right)=12 a(8 a+1) P_{427}(a, c),
$$

so, again, it suffices to show $P_{427}(a, c)<0$. But now we observe that $\partial_{c} P_{427}(a, c)=192 a^{2}-$ $1824 a+2850$ is positive for $\varrho_{421} \leqslant a<\frac{1}{8}(38-\sqrt{494})$, and non-positive for $\frac{1}{8}(38-\sqrt{494}) \leqslant$ $a \leqslant \frac{22}{10}$. By (7.18) again, in the first case, we have

$$
\begin{aligned}
P_{427}(a, c) & \leqslant P_{427}\left(a, \frac{1}{512}\left(-1536 a^{3}+1728 a^{2}-424 a+25\right)\right) \\
& =\frac{1}{256}(8 a+1)\left(6144 a^{4}-62976 a^{3}+174496 a^{2}-172672 a+35625\right)<0,
\end{aligned}
$$

and in the second case

$$
\begin{aligned}
P_{427}(a, c) & \leqslant P_{427}\left(a, \frac{1}{192}\left(-768 a^{3}+768 a^{2}-160 a+7\right)\right) \\
& =\frac{19}{32}(8 a+1)\left(256 a^{2}-648 a+175\right)<0 .
\end{aligned}
$$

REMARK 7.4. Mathematica tells us that among the integers $4 \leqslant k \leqslant 1000$, exactly members of the interval $77 \leqslant k \leqslant 98$ share the property that $\lambda(k, a, c, d)<0$ for any allowed $a, c$ and $d$ in the previous lemma.

Lemma 7.5. For each $\frac{1}{24}(9+2 \sqrt{6}) \leqslant a \leqslant \frac{22}{10}$, and any $c$ and $d$ satisfying (7.19), we have that $\lambda(54, a, c, d)<0$.

Proof. The proof is completely analogous to the previous one. Now $\partial_{d} \lambda(54, a, c, d)=$ $-24 P_{428}(a)$ has two roots $\varrho_{422} \approx 0.808208$ and $\varrho_{423} \approx 1.97947$ in the given $a$-interval, so we again separate two cases.

The interval $a \in\left[\frac{1}{24}(9+2 \sqrt{6}), \varrho_{422}\right) \cup\left(\varrho_{423}, \frac{22}{10}\right]$. Now $\partial_{d} \lambda(54, a, c, d)>0$, so by $(7.19)$

$$
\begin{aligned}
\lambda(54, a, c, d) & \leqslant \lambda\left(54, a, c, \frac{1}{2048}\left(768 a^{3}-512 a^{2}-512 a c+104 a+192 c-7\right)\right) \\
& =\frac{159}{256}(8 a+1) P_{429}(a, c) .
\end{aligned}
$$

We show that $P_{429}<0$. Indeed, $\partial_{c} P_{429}(a, c)=64\left(192 a^{2}-832 a+663\right)$ is positive for $a \in$ $\left[\frac{1}{24}(9+2 \sqrt{6}), \varrho_{422}\right)$, so by $(7.19)$ here

$$
\begin{aligned}
P_{429}(a, c) & \leqslant P_{429}\left(a, \frac{1}{128}\left(192 a^{2}-104 a+11\right)\right) \\
& =\frac{1}{2}(8 a+1)\left(12288 a^{4}-58368 a^{3}+76160 a^{2}-35152 a+4199\right)<0 .
\end{aligned}
$$


On the other hand, $\partial_{c} P_{429}(a, c)<0$ for $a \in\left(\varrho_{423}, \frac{22}{10}\right]$, hence by $(7.19)$ we now have

$$
\begin{aligned}
P_{429}(a, c) & \leqslant P_{429}\left(a, \frac{1}{512}\left(-1536 a^{3}+1728 a^{2}-424 a+25\right)\right) \\
& =\frac{1}{8}(8 a+1)\left(12288 a^{4}-46080 a^{3}+53888 a^{2}-29328 a+4199\right)<0 .
\end{aligned}
$$

The interval $a \in\left[\varrho_{422}, \varrho_{423}\right]$. Here $\partial_{d} \lambda(54, a, c, d) \leqslant 0$, so by $(7.19)$

$$
\begin{aligned}
\lambda(54, a, c, d) & \leqslant \lambda\left(54, a, c, \frac{1}{4096}\left(-384 a^{2}+224 a+512 c-25\right)\right) \\
& =\frac{3}{512}(8 a+1) P_{4210}(a, c) .
\end{aligned}
$$

By examining the sign of $\partial_{c} P_{4210}(a, c)=-512\left(256 a^{3}-5088 a^{2}+16536 a-11713\right)$, we prove finally that $P_{4210}<0$ as well. We see that $\partial_{c} P_{4210}(a, c)>0$ for $\varrho_{422} \leqslant a<\varrho_{424} \approx 1.00126$, but $\partial_{c} P_{4210}(a, c) \leqslant 0$ for $\varrho_{424} \leqslant a \leqslant \varrho_{423}$. By taking into account $(7.19)$, in the first case we have

$$
\begin{aligned}
P_{4210}(a, c) & \leqslant P_{4210}\left(a, \frac{1}{128}\left(192 a^{2}-104 a+11\right)\right) \\
& =53(8 a+1)\left(12288 a^{4}-58368 a^{3}+76160 a^{2}-35152 a+4199\right)<0
\end{aligned}
$$

while in the second case

$$
\begin{aligned}
P_{4210}(a, c) & \leqslant P_{4210}\left(a, \frac{1}{512}\left(-1536 a^{3}+1728 a^{2}-424 a+25\right)\right) \\
& =8 a(8 a+1)\left(6144 a^{4}-45312 a^{3}+102368 a^{2}-86284 a+17225\right)<0 .
\end{aligned}
$$

Remark 7.6. According to Mathematica, $k=54$ is the only integer in the interval $[4,1000] \cap \mathbb{N}$ such that $\lambda(k, a, c, d)<0$ holds for any admissible $a, c$ and $d$ triples in the previous lemma.

Lemma 7.7. For each $\frac{22}{10}<a$, and any $c$ and $d$ satisfying (7.18), there exists an integer $k \geqslant 4$ such that $\lambda(k, a, c, d)<0$.

Proof. Let us fix any admissible $a, c$ and $d$ throughout the proof. We first compute $\partial_{d} \lambda(k, a, c, d)=2 P_{4211}(k, a)$, where

$$
P_{4211}(k, a)=k^{3}-(96 a+6) k^{2}+\left(2304 a^{2}+288 a+11\right) k-12288 a^{3}-2304 a^{2}-192 a-6,
$$

see Figure 9, and show that this cubic polynomial (in $k$ ) has three distinct real roots and also give some bounds on the roots in terms of $a$.

It is seen that the function $k \mapsto \partial_{k} P_{4211}(k, a)$ has two distinct real roots at $k=\varrho_{425}^{ \pm}(a):=$ $\frac{1}{3}\left(96 a+6 \pm \sqrt{3} \sqrt{768 a^{2}+96 a+1}\right)$, being the abscissae of the two strict local extrema of $k \mapsto$ $P_{4211}(k, a)$. It is also easily established that $P_{4211}\left(\varrho_{425}^{-}(a), a\right)>0$ and $P_{4211}\left(\varrho_{425}^{+}(a), a\right)<0$. By observing that $P_{4211}(4, a)<0$ and $4<\varrho_{425}^{-}(a)$, further, by taking into account that the leading coefficient of $k \mapsto P_{4211}(k, a)$ is positive, we get that each of the three disjoint intervals

$$
\left(4, \varrho_{425}^{-}(a)\right) \cup\left(\varrho_{425}^{-}(a), \varrho_{425}^{+}(a)\right) \cup\left(\varrho_{425}^{+}(a),+\infty\right)
$$

contains precisely one root of $k \mapsto P_{4211}(k, a)=\frac{1}{2} \partial_{d} \lambda(k, a, c, d)$.

By denoting these roots by $\varrho_{426}(a)_{1}<\varrho_{426}(a)_{2}<\varrho_{426}(a)_{3}$, we also see from the properties of the cubic polynomial that for $k \in\left(\varrho_{426}(a)_{2}, \varrho_{426}(a)_{3}\right), \partial_{d} \lambda(k, a, c, d)<0$. (Note that for any such $k, k \geqslant 4$ holds.) Also referring to (7.18), this means that for $k \in\left(\varrho_{426}(a)_{2}, \varrho_{426}(a)_{3}\right)$ we have

$$
\lambda(k, a, c, d) \leqslant \lambda\left(k, a, c, \frac{1}{2}\left(-6 a^{4}+6 a^{3}-a^{2}-2 a c\right)\right)=6 a(8 a+1) P_{4212}(k, a, c),
$$




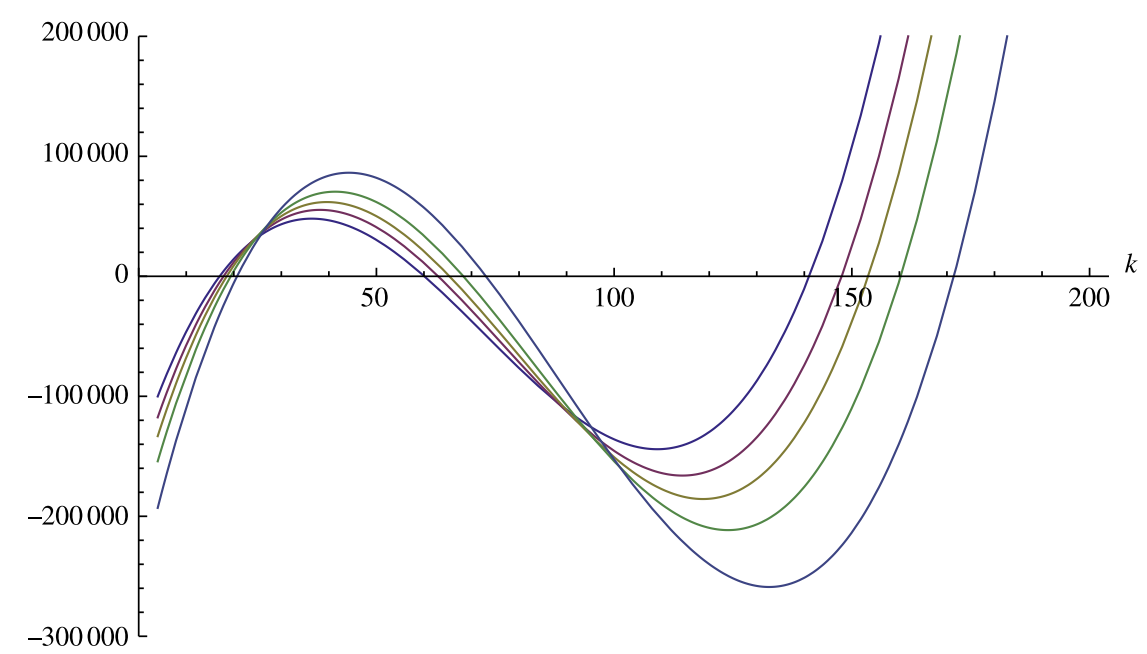

Figure 9. Graphs of the functions $k \mapsto P_{4211}(k, a)$ for some values of the parameter $a>\frac{22}{10}$ in the $\widehat{\Pi}_{4 / 4,2}$ class. Each curve has three real roots, denoted by $\varrho_{426}(a)_{1}<\varrho_{426}(a)_{2}<\varrho_{426}(a)_{3}$, and two local extrema, denoted by $\varrho_{425}^{-}(a)<\varrho_{425}^{+}(a)$.

where

$$
\begin{aligned}
P_{4212}(k, a, c)= & k^{2}\left(4 a^{3}-5 a^{2}+a+c\right)+k\left(-192 a^{4}+212 a^{3}-27 a^{2}-48 a c-2 a-3 c\right) \\
& +1536 a^{5}-1344 a^{4}+104 a^{3}+384 a^{2} c+18 a^{2}+48 a c+a+2 c .
\end{aligned}
$$

Clearly, in order to finish the proof, it is sufficient to show that there is an integer $k \in$ $\left(\varrho_{426}(a)_{2}, \varrho_{426}(a)_{3}\right)$ such that $P_{4212}(k, a, c)<0$.

Let the two roots of $k \mapsto \partial_{c} P_{4212}(k, a, c)=k^{2}-(48 a+3) k+384 a^{2}+48 a+2$ be denoted by $\varrho_{427}^{ \pm}(a):=\frac{1}{2}\left(48 a+3 \pm \sqrt{768 a^{2}+96 a+1}\right)$, then $\partial_{c} P_{4212}(k, a, c)<0$ for $k \in\left(\varrho_{427}^{-}(a), \varrho_{427}^{+}(a)\right)$. We will show next that

$$
\left(\varrho_{426}(a)_{2}, \varrho_{426}(a)_{3}\right) \cap\left(\varrho_{427}^{-}(a), \varrho_{427}^{+}(a)\right)=\left(\varrho_{426}(a)_{2}, \varrho_{427}^{+}(a)\right) .
$$

In fact, we have seen in $(7.21)$ that $\varrho_{425}^{-}(a)<\varrho_{426}(a)_{2}$, and an elementary calculation shows that $\varrho_{427}^{-}(a)<\varrho_{425}^{-}(a)$. An analogous argument tells us that $\varrho_{427}^{+}(a)<\varrho_{425}^{+}(a)<\varrho_{426}(a)_{3}$, verifying (7.22).

Thus, by picking any $k \in\left(\varrho_{426}(a)_{2}, \varrho_{427}^{+}(a)\right)$, we can conclude (with the help of (7.18) also) that

$$
\begin{aligned}
P_{4212}(k, a, c) & \leqslant P_{4212}\left(k, a, \frac{1}{192}\left(-768 a^{3}+768 a^{2}-160 a+7\right)\right) \\
& =\frac{1}{192}(8 a+1)(k-1)\left((7-24 a) k+768 a^{2}-96 a-14\right),
\end{aligned}
$$

from which we see that the proof is finished if we find any $k \in\left(\varrho_{426}(a)_{2}, \varrho_{427}^{+}(a)\right) \cap \mathbb{N}$ such that $(7-24 a) k+768 a^{2}-96 a-14<0$, or, in other words, $k>\left(768 a^{2}-96 a-14\right) /(24 a-7)$.

We aim to show now that $\varrho_{426}(a)_{2}<\left(768 a^{2}-96 a-14\right) /(24 a-7)$. (Notice that this time both $\varrho_{426}(a)_{2}<\varrho_{425}^{+}(a)$ and $\left(768 a^{2}-96 a-14\right) /(24 a-7)<\varrho_{425}^{+}(a)$ are true, so we have to take an extra step.) But by referring to $(7.21)$ we have $\varrho_{426}(a)_{1}<\varrho_{425}^{-}(a)$, and an elementary computation yields that $\varrho_{425}^{-}(a)<\left(768 a^{2}-96 a-14\right) /(24 a-7)$, further, it is not hard to see that

$$
P_{4211}\left(\frac{768 a^{2}-96 a-14}{24 a-7}, a\right)=-\frac{48 a(8 a+1)(16 a-3)\left(9216 a^{3}-1512 a+49\right)}{(24 a-7)^{3}}<0 .
$$


However, we already know that $P_{4211}(\cdot, a)<0$ if and only if $k \in\left(-\infty, \varrho_{426}(a)_{1}\right) \cup$ $\left(\varrho_{426}(a)_{2}, \varrho_{426}(a)_{3}\right)$, so these force $\varrho_{426}(a)_{2}<\left(768 a^{2}-96 a-14\right) /(24 a-7)$.

Summarizing, it is enough to prove as a last step that $\varnothing \neq\left(\left(768 a^{2}-96 a-14\right) /(24 a-7)\right.$, $\left.\varrho_{427}^{+}(a)\right) \cap \mathbb{N}$, being true, since $\varrho_{427}^{+}(a)-\left(768 a^{2}-96 a-14\right) /(24 a-7)>9$ for $a>\frac{22}{10}$.

REMARK 7.8. Similarly to Remark 6.5 , the bound 9 could not be replaced by, say, 10 on $a \in(22 / 10,+\infty)$.

REMARK 7.9. The proof we have just presented for Lemma 7.7 (as well as the one we will present for the next lemma) would break down on the larger interval $a \geqslant(9+2 \sqrt{6}) / 24$. This explains why we have chosen the finer subdivision $a \in\left[\frac{1}{24}(9+2 \sqrt{6}), \frac{22}{10}\right] \cup\left(\frac{22}{10},+\infty\right)$.

Lemma 7.10. For each $\frac{22}{10}<a$, and any $c$ and $d$ satisfying (7.19), there is an integer $k \geqslant 4$ such that $\lambda(k, a, c, d)<0$.

Proof. We have seen in the preceding proof that $\partial_{d} \lambda(k, a, c, d) \geqslant 0$ for $k \in\left[\varrho_{426}(a)_{1}, \varrho_{426}(a)_{2}\right]$ $\subset(4,+\infty)$, so, by (7.19), for these $k$ values we have

$$
\begin{aligned}
\lambda(k, a, c, d) & \leqslant \lambda\left(k, a, c, \frac{1}{2048}\left(768 a^{3}-512 a^{2}-512 a c+104 a+192 c-7\right)\right) \\
& =\frac{(8 a+1)(k-1)}{1024} P_{4213}(k, a, c),
\end{aligned}
$$

where

$$
\begin{aligned}
P_{4213}(k, a, c)= & k^{2}\left(768 a^{3}-768 a^{2}+160 a+192 c-7\right) \\
& +k\left(-49152 a^{4}+39168 a^{3}-5376 a^{2}-12288 a c-128 a-960 c+35\right) \\
& +589824 a^{5}-294912 a^{4}+29184 a^{3}+147456 a^{2} c+3840 a^{2} \\
& +24576 a c-384 a+1152 c-42 .
\end{aligned}
$$

To show that $P_{4213}(k, a, c)<0$ for a suitable $k \in\left[\varrho_{426}(a)_{1}, \varrho_{426}(a)_{2}\right] \cap \mathbb{N}$, we first compute

$$
\partial_{c} P_{4213}(k, a, c)=192\left(k^{2}-(64 a+5) k+768 a^{2}+128 a+6\right),
$$

and denote the roots of $k \mapsto \partial_{c} P_{4213}(k, a, c)$ by $\varrho_{428}^{ \pm}(a):=\frac{1}{2}\left(64 a+5 \pm \sqrt{1024 a^{2}+128 a+1}\right)$. Note that $\partial_{c} P_{4213}(k, a, c)<0$ for $\varrho_{428}^{-}(a)<k<\varrho_{428}^{+}(a)$. Now by using formulae (7.21) and (7.20), one easily shows that $\varrho_{425}^{-}(a)<\varrho_{428}^{-}(a)<\varrho_{425}^{+}(a)$ and

$$
P_{4211}\left(\varrho_{428}^{-}(a), a\right)=32 a(8 a+1)\left(\sqrt{1024 a^{2}+128 a+1}-16 a-1\right)>0,
$$

implying $\varrho_{426}(a)_{1}<\varrho_{428}^{-}(a)<\varrho_{426}(a)_{2}$. Hence if $k \in\left(\varrho_{428}^{-}(a), \varrho_{426}(a)_{2}\right] \cap \mathbb{N}$, then, also by (7.19),

$$
P_{4213}(k, a, c) \leqslant P_{4213}\left(k, a, \frac{1}{512}\left(-1536 a^{3}+1728 a^{2}-424 a+25\right)\right)=\frac{1}{8}(8 a+1) P_{4214}(k, a),
$$

with

$$
\begin{aligned}
P_{4214}(k, a)= & k^{2}\left(192 a^{2}-144 a+19\right)+k\left(-12288 a^{3}+2112 a^{2}+1296 a-95\right) \\
& +147456 a^{4}+110592 a^{3}-27264 a^{2}-2016 a+114 .
\end{aligned}
$$

Now the leading coefficient, $192 a^{2}-144 a+19$ of $k \mapsto P_{4214}(k, a)$ is positive, and its discriminant, $\Delta_{42}(a):=37748736 a^{6}-51904512 a^{5}+46043136 a^{4}-14751744 a^{3}+2101632 a^{2}-27360 a+361$ 
is also positive. (This last statement can easily be shown by noticing that $\Delta_{42}^{\prime \prime \prime \prime}(a)>0$ for all $a \in \mathbb{R}$, and $\Delta_{42}^{\prime \prime \prime}\left(\frac{22}{10}\right)>0$, so $\Delta_{42}^{\prime \prime \prime}(a)>0$ for $a>\frac{22}{10}$. Repeating this recursively and similarly for the lower order derivatives we get that $\Delta_{42}(a)>0$ for all $a>\frac{22}{10}$.) This means that we can denote by $\varrho_{429}^{ \pm}(a)$ the two real roots of the quadratic polynomial $k \mapsto P_{4214}(k, a)$. Clearly, $\varrho_{429}^{-}(a)<k<\varrho_{429}^{+}(a)$ implies $P_{4214}(k, a)<0$.

In light of the above, in order to finish the proof it is enough to show that

$$
\varnothing \neq\left(\varrho_{429}^{-}(a), \varrho_{429}^{+}(a)\right) \cap\left(\varrho_{428}^{-}(a), \varrho_{426}(a)_{2}\right] \cap \mathbb{N} .
$$

More specifically, we can prove that the following sufficient condition

$$
\varrho_{428}^{-}(a)<\varrho_{429}^{-}(a)<16 a+19<16 a+24<\varrho_{426}(a)_{2}<\varrho_{429}^{+}(a)
$$

holds: the first inequality is true because of

$$
P_{4214}\left(\varrho_{428}^{-}(a), a\right)=128 a\left((24 a-7) \sqrt{1024 a^{2}+128 a+1}+136 a+7\right)>0
$$

and

$$
\partial_{k} P_{4214}\left(\varrho_{428}^{-}(a), a\right)=-6144 a^{2}+1792 a-\left(192 a^{2}-144 a+19\right) \sqrt{1024 a^{2}+128 a+1}<0 ;
$$

the second inequality is a consequence of

$$
P_{4214}(16 a+19, a)=-16\left(576 a^{3}-1264 a^{2}+1209 a-323\right)<0 ;
$$

the fourth inequality follows from $P_{4211}(16 a+24, a)=4096 a^{3}+768 a^{2}-25360 a+10626>0$ and $16 a+24<\varrho_{425}^{+}(a)$; while the fifth inequality is derived from the fact that

$$
\varrho_{425}^{-}(a)<\varrho_{429}^{+}(a)=\frac{12288 a^{3}-2112 a^{2}-1296 a+95+\sqrt{\Delta_{42}(a)}}{2\left(192 a^{2}-144 a+19\right)}
$$

and

$$
\begin{aligned}
P_{4211}\left(\varrho_{429}^{+}(a), a\right)=- & \frac{96 a}{\left(192 a^{2}-144 a+19\right)^{3}} \\
\times & \left(301989888 a^{8}-622854144 a^{7}+474218496 a^{6}\right. \\
& -1089110016 a^{5}+816156672 a^{4} \\
& -250172928 a^{3}+31469760 a^{2}-960184 a+9025 \\
+ & \left(98304 a^{5}-135168 a^{4}-132096 a^{3}+106880 a^{2}\right. \\
& \left.-19464 a+475) \sqrt{\Delta_{42}(a)}\right)<0 .
\end{aligned}
$$

REMARK 7.11. In the proof of this lemma, in order to establish $\lambda(k, a, c, d)<0$, one would have four initial possibilities to choose from according to the signs of the appropriate $\partial_{d}$ and $\partial_{c}$ partial derivatives. It turns out that if one chooses a certain two out of these four possibilities, then the proof could not be completed. On the other hand, the remaining two feasible directions (out of which we have presented one) require approximately the same amount and type of computations.

REMARK 7.12. The motivation for (7.23) came from the fact that

$$
\lim _{a \rightarrow+\infty} \frac{\varrho_{429}^{-}(a)}{a}=16<\lim _{a \rightarrow+\infty} \frac{\varrho_{426}(a)_{2}}{a}=\operatorname{root}_{2}(1,-96,2304,-12288) \approx 26.4432 .
$$

In fact, for $a>\frac{22}{10}$, we have $\varrho_{426}(a)_{2}-\varrho_{429}^{-}(a)>6$, but $\ldots>7$ does not hold. 


\section{Further results and questions}

In this section we present some intermediate results that may prove useful in future studies of the radius of absolute monotonicity.

The motivation for $\S 8.1$ came from Conjecture 1.5. Let $A, b$ denote the coefficients of an arbitrary RK method; then $R(A, b)>0 \Longrightarrow A \geqslant 0$ (again, matrix inequalities are understood componentwise). The trace inequality (8.3) for non-negative matrices is used to derive a certain non-linear relation (8.4) between the first few coefficients of the polynomial appearing in the numerator of the stability function (1.1).

Next we turn our attention to Conjecture 1.6. Let us fix $s \geqslant 3$ and $p=2$, choose an arbitrary SDIRK method with coefficients $A, b$, and consider its stability function $\psi \in \widehat{\Pi}_{s / s, 2}$ as described by formula (2.5). Let $a_{0}, a_{1}$ and $a_{2}$ denote the first few coefficients of the numerator of $\psi$, that is $a_{0}:=1, a_{1}:=1-a\left(\begin{array}{l}s \\ 1\end{array}\right)$ and $a_{2}:=\frac{1}{2}-a\left(\begin{array}{l}s \\ 1\end{array}\right)+a^{2}\left(\begin{array}{l}s \\ 2\end{array}\right)$, then a simple computation shows that (8.4) is satisfied, in fact, with equality, despite the fact that the non-negativity condition $A \geqslant 0$ here is not assumed. For $3 \leqslant s \leqslant 8$, Lemma 8.7 in $\oint 8.2$ gives a remarkable uniqueness result for polynomials: the unique polynomial given by Lemma 8.7 is identical to the numerator of the conjectured optimal and unique $\psi$ function appearing in Theorem 2.2 (or in Conjecture 1.6). Apart from the fact that we could prove Lemma 8.7 only for $3 \leqslant s \leqslant 8$, the missing link is the following: the uniqueness result in Theorem 2.2 is essentially obtained under the condition

$$
\psi^{(k)}(-2 s) \geqslant 0 \quad \text { for } k \in \mathbb{N},
$$

whereas in Lemma 8.7 we assumed

$$
P^{(k)}(-2 s) \geqslant 0 \quad \text { for } k=0,1, \ldots, s .
$$

We are very curious whether a result similar to Lemma 8.7 could lead to a proof of Theorem 2.2 for general $s$ values, that is, to a proof of Conjecture 1.6.

In $\oint 8.3$ we give a formula for the elements of the $\widehat{\Pi}_{s / s, p}$ class for general $s$ and $p$ values, together with some additional remarks and questions.

REMARK 8.1. We have found the following conjecture whose assumption, with $p_{m}=$ $P^{(m)}(x) / m !, q=Q(x)$ and $\widetilde{q}=Q^{\prime}(x)=-a$ for some $x \in[-2 s, 0]$, is similar to the sum in (2.7) apart from the factor $(-1)^{k-m}$ and the fact that the $p_{m}$ quantities can be independent of one another. The conjecture has been proved by Mathematica for $1 \leqslant s \leqslant 10$, and we have a 'manual' proof for $s=2$. We do not know whether the conjecture could be used in a transition from conditions (8.1) to (8.2).

Conjecture 8.2. Fix any $s \in \mathbb{N}^{+}, 0 \neq q \in \mathbb{R}, 0<\widetilde{q} \in \mathbb{R}$ and $p_{m} \in \mathbb{R}(m=0,1, \ldots, s+1)$, and suppose that for each $k=0,1, \ldots, s+1$ we have

$$
\sum_{m=0}^{\min (k, s)}\left(\begin{array}{c}
s-1+k-m \\
s-1
\end{array}\right) \cdot p_{m} \cdot q^{m} \cdot(-\widetilde{q})^{k-m} \geqslant 0 .
$$

Then $p_{0} \geqslant 0$ and for $m=1,2, \ldots, s+1$, we have $p_{m}=p_{0}\left(\begin{array}{c}s \\ m\end{array}\right) \cdot\left(\frac{\widetilde{q}}{q}\right)^{m}$. Moreover, the above sum is equal to $p_{0}$ for $k=0$, and to 0 for $k=1,2, \ldots, s+1$.

8.1. Trace inequalities for non-negative matrices and the numerator of the stability function

Conjecture 1.5 in the $s=3, p=2$ case claims that $R(A, b) \leqslant 6$ for any RK method with coefficients $A, b$. The following general lemma was discovered while investigating this conjectured bound. Due to the remark in the beginning of $\S 8, A \geqslant 0$ will be assumed throughout the current $\S 8.1$. 
Let us fix a positive integer $s \geqslant 2$ and apply the following notation: if $A$ is an $s$-by-s matrix, then $\tau:=\operatorname{tr}(A)$ and $\tau_{k}:=\operatorname{tr}\left(A^{k}\right)$ for any integer $k \geqslant 2$.

Lemma 8.3. Fix a positive integer $n \geqslant 2$ and suppose that $A$ is an $s$-by-s (componentwise) non-negative matrix. Then

$$
s^{n-1} \tau_{n} \geqslant \tau^{n} .
$$

Moreover, in the $n=2$ case equality holds if and only if $a_{k, k}=a_{1,1}$ for $k=2,3, \ldots, s$ and $a_{i, j} a_{j, i}=0$ for all $i \neq j$, further, if $n \geqslant 3$ and $s^{n-1} \tau_{n}=\tau^{n}$, then $a_{k, k}=a_{1,1}$ for $k=2,3, \ldots, s$.

Proof. If $A$ and $B$ are non-negative $s$-by-s matrices, then $(A B)_{k, k} \geqslant a_{k, k} b_{k, k}$. Applying this recursively, we get that $\operatorname{tr}\left(A^{n}\right) \geqslant \sum_{k=1}^{s} a_{k, k}^{n}$. Then the inequality between the $n$th power mean and the arithmetic mean shows that

$$
\sqrt[n]{\frac{\operatorname{tr}\left(A^{n}\right)}{s}} \geqslant \sqrt[n]{\frac{\sum_{k=1}^{s} a_{k, k}^{n}}{s}} \geqslant \frac{\sum_{k=1}^{s} a_{k, k}}{s}=\frac{\operatorname{tr}(A)}{s},
$$

proving the trace inequalities. Now if $n \geqslant 3$ and $s^{n-1} \tau_{n}=\tau^{n}$, then we have equality in the power mean inequality, which implies $a_{k, k}=a_{1,1}$ for $k=2,3, \ldots, s$. Finally, suppose that $n=2$. Then $s \tau_{2}=\tau^{2}$ holds if and only if we have equality in the power mean inequality and $\operatorname{tr}\left(A^{2}\right)=\sum_{k=1}^{s} a_{k, k}^{2}$. But the former holds if and only if $a_{k, k}=a_{1,1}$ for $k=2,3, \ldots, s$, while the latter holds if and only if $a_{i, j} a_{j, i}=0$ for all $i \neq j$.

\section{REMARK 8.4.}

- If $s=2, n=3, a_{1,1}=a_{2,2}=0$ and $a_{1,2}=a_{2,1}=1$, then $s^{n-1} \tau_{n}=\tau^{n}$, but $a_{1,2} a_{2,1} \neq 0$.

- The constant $s^{n-1}$ is the best possible (as shown by $A=I$ ).

- We cannot expect a 'converse' trace inequality, since for the matrix $A$ with zeros in the diagonal and with all other entries 1 , we have $\operatorname{tr}^{2}(A)=0$ and $\operatorname{tr}\left(A^{2}\right)>0$.

- For the conjectured optimal RK method satisfying $R(A, b)=6$, we have equality in (8.3) for $n=2$.

Let us now fix any $s \geqslant 3$. By repeatedly using the formulae for the derivative of the determinant and the trace

$$
(\operatorname{det} \Phi(\cdot))^{\prime}=(\operatorname{det} \Phi(\cdot)) \cdot \operatorname{tr}\left(\Phi^{-1}(\cdot) \Phi^{\prime}(\cdot)\right) \quad \text { and } \quad(\operatorname{tr} \Phi(\cdot))^{\prime}=\operatorname{tr}\left(\Phi^{\prime}(\cdot)\right),
$$

where $\Phi: \mathbb{R} \rightarrow \mathbb{R}^{s \times s}$ is a smooth, and for the first formula, invertible, matrix function, the cyclic invariance of the trace together with the $p=2$ order conditions, we see that the numerator of the stability function (1.1), $\operatorname{det}\left(I-z A+z \mathbb{1} b^{\top}\right)$, can be written in the form

$$
P(z)=1+(1-\tau) z+\frac{1}{2}\left((1-\tau)^{2}-\tau_{2}\right) z^{2}+\sum_{k=3}^{s} a_{k} z^{k},
$$

with suitable real parameters $a_{k}$ (there may be further restrictions on the $a_{k}$ parameters which are ignored here). Let us introduce $a_{0}:=1, a_{1}:=1-\tau, a_{2}:=\frac{1}{2}\left(a_{1}^{2}-\tau_{2}\right)$. Then, due to $A \geqslant 0$, we have $\tau \geqslant 0$ and $\tau_{2} \geqslant 0$, so (8.3) with $n=2$ implies $-\tau_{2} \leqslant-\tau^{2} / s$, thus $a_{2}=\frac{1}{2}\left(a_{1}^{2}-\tau_{2}\right) \leqslant \frac{1}{2}\left(a_{1}^{2}-\tau^{2} / s\right)$, but $\tau=1-a_{1}$, hence we have derived a non-linear condition

$$
\frac{1}{2}\left(a_{1}^{2}-\frac{\left(1-a_{1}\right)^{2}}{s}\right) \geqslant a_{2} .
$$


8.2. Uniqueness results for polynomials closely related to the class $\widehat{\Pi}_{s / s, 2}$

In this section, the main lemma is Lemma 8.7, although Lemma 8.5 can be of independent interest, giving lower and upper bounds on the coefficients of a general polynomial $P$ with $P(0)=1$ that is absolutely monotonic at a point $-r<0$, together with a uniqueness result.

Lemma 8.5. Let $s$ denote a fixed positive integer and set $P(z):=1+\sum_{n=1}^{s} a_{n} z^{n}$ with some real coefficients $a_{n}$. Fix any $r>0$ and suppose that $P^{(k)}(-r) \geqslant 0$ for all $k=0,1, \ldots, s$. Then for each index $1 \leqslant n \leqslant s$ we have $0 \leqslant a_{n} \leqslant\left(\begin{array}{l}s \\ n\end{array}\right) / r^{n}$. Moreover, if there is at least one $n$ $(1 \leqslant n \leqslant s)$ with $a_{n}=\left(\begin{array}{l}s \\ n\end{array}\right) / r^{n}$, then $P(z)=(1+z / r)^{s}$.

Proof. By assumption, with $\gamma_{k}:=\left(P^{(k)}(-r) / k !\right) r^{k}$ we have $\gamma_{k} \geqslant 0$ for $k=0,1, \ldots, s$. Taylor expansion, the binomial theorem and interchanging the order of summations show that

$$
\begin{aligned}
P(z) & =\sum_{k=0}^{s} \gamma_{k}\left(1+\frac{z}{r}\right)^{k}=\sum_{k=0}^{s} \sum_{n=0}^{k} \gamma_{k}\left(\begin{array}{l}
k \\
n
\end{array}\right) \frac{z^{n}}{r^{n}} \\
& =\sum_{n=0}^{s} \sum_{k=n}^{s} \gamma_{k}\left(\begin{array}{l}
k \\
n
\end{array}\right) \frac{z^{n}}{r^{n}}=\sum_{n=0}^{s}\left(\frac{1}{r^{n}} \sum_{k=n}^{s} \gamma_{k}\left(\begin{array}{l}
k \\
n
\end{array}\right)\right) z^{n} .
\end{aligned}
$$

Now fix $1 \leqslant n \leqslant s$. By equating the coefficients of $z^{n}$ we get $a_{n}=\left(1 / r^{n}\right) \sum_{k=n}^{s} \gamma_{k}\left(\begin{array}{l}k \\ n\end{array}\right)$, and, from the equality of the constant terms

$$
\sum_{k=0}^{s} \gamma_{k}=1
$$

Non-negativity of the $\gamma_{k}$ coefficients implies $a_{n} \geqslant 0$ and (8.5) shows $0 \leqslant \gamma_{k} \leqslant 1$ for all $0 \leqslant k \leqslant s$. Finally we seek the maximum of $\sum_{k=n}^{s} \gamma_{k}\left(\begin{array}{l}k \\ n\end{array}\right)$ knowing $0 \leqslant \gamma_{k}$ and (8.5). Suppose that $\gamma_{m}>0$ for some $m$ with $0 \leqslant m \leqslant s-1$. Then we define $\widetilde{\gamma_{s}}:=\gamma_{s}+\gamma_{m}, \widetilde{\gamma_{m}}:=0$ and $\widetilde{\gamma_{k}}:=\gamma_{k}$ for $s \neq k \neq m$. Clearly, $0 \leqslant \widetilde{\gamma_{k}}$ for each $0 \leqslant k \leqslant s$ and $\sum_{k=0}^{s} \widetilde{\gamma_{k}}=1$, but (by using the convention that $\left(\begin{array}{c}m \\ n\end{array}\right)=0$ for $m<n$ )

$$
\sum_{k=n}^{s} \widetilde{\gamma_{k}}\left(\begin{array}{l}
k \\
n
\end{array}\right)-\sum_{k=n}^{s} \gamma_{k}\left(\begin{array}{l}
k \\
n
\end{array}\right)=\left(\begin{array}{l}
s \\
n
\end{array}\right) \gamma_{m}+\left(\begin{array}{l}
m \\
n
\end{array}\right)\left(-\gamma_{m}\right)=\gamma_{m}\left(\left(\begin{array}{l}
s \\
n
\end{array}\right)-\left(\begin{array}{l}
m \\
n
\end{array}\right)\right)
$$

and this last expression is strictly positive, because $1 \leqslant n \leqslant s$ is fixed, $s>m$ and $\gamma_{m}>0$. We can therefore conclude that (for fixed $s \geqslant 1$ and $1 \leqslant n \leqslant s$ ) the value of $\sum_{k=n}^{s} \gamma_{k}\left(\begin{array}{l}k \\ n\end{array}\right)$ over all $0 \leqslant \gamma_{k}(k=0,1, \ldots, s)$ and under condition (8.5) is maximal if and only if $\gamma_{0}=\gamma_{1}=\ldots=$ $\gamma_{s-1}=0$ and $\gamma_{s}=1$. This property establishes the upper bound on $a_{n}$ and the uniqueness part as well.

Next we present an interesting lemma about representing a certain linear combination of the three lowest order coefficients of a general polynomial of degree at most $s$ in terms of another linear combination of its derivatives evaluated at $-2 s$.

Lemma 8.6. Fix any integer $s \geqslant 2$ and choose an arbitrary $P(z):=1+\sum_{k=1}^{s} a_{k} z^{k}$ (with complex coefficients). Then

$$
\sum_{k=0}^{s} \frac{(2 s)^{k}(s-k)(s-k-1)}{k ! s(s-1)} P^{(k)}(-2 s)=1-4 a_{1}+\frac{8 s}{s-1} a_{2} .
$$


Proof. Direct comparison of the $a_{m}$ coefficients on both sides by using some simple binomial identities (that can be obtained, for example, after (successively) differentiating the binomial expansion of $\left.x \mapsto(1+x)^{n}\right)$.

The preceding two lemmas are used in proving the following uniqueness result in a class of polynomials of degree at most $s$ (unfortunately only in a restricted range $s \leqslant 8$ ) which are absolutely monotonic at $-2 s$ and whose lowest order coefficients satisfy a certain non-linear relation. The origin of condition (8.6) is of course inequality (8.4) in a slightly different context.

Lemma 8.7. Let us pick an integer $s$ with $3 \leqslant s \leqslant 8$, and set $P(z):=1+\sum_{k=1}^{s} a_{k} z^{k}$ with some real coefficients $a_{k}$. Suppose $P^{(k)}(-2 s) \geqslant 0$ for all $k=0,1, \ldots, s$, further, that

$$
\frac{1}{2}\left(a_{1}^{2}-\frac{\left(1-a_{1}\right)^{2}}{s}\right) \geqslant a_{2} .
$$

Then $P(z)=(1+z / 2 s)^{s}$.

Proof. By Lemma 8.5 with $r=2 s$, we have $a_{n} \geqslant 0$ for all $n=1,2, \ldots, s$. On the other hand, since now $P^{(k)}(-2 s) \geqslant 0$ by assumption, by applying Lemma 8.6 we get that

$$
1-4 a_{1}+\frac{8 s}{s-1} a_{2} \geqslant 0
$$

From this inequality we estimate $a_{2}$ as $a_{2} \geqslant((s-1) / 8 s)\left(4 a_{1}-1\right)$, so $\frac{1}{2}\left(a_{1}^{2}-\left(1-a_{1}\right)^{2} / s\right) \geqslant$ $((s-1) / 8 s)\left(4 a_{1}-1\right)$, which can be factorized as

$$
\frac{\left(2 a_{1}-1\right)\left(2 a_{1}(s-1)+5-s\right)}{8 s} \geqslant 0,
$$

showing (by using $s>1$ only) that either

$$
a_{1} \leqslant \frac{1}{2} \quad \text { and } \quad a_{1} \leqslant \frac{s-5}{2(s-1)}
$$

or

$$
a_{1} \geqslant \frac{1}{2} \quad \text { and } \quad a_{1} \geqslant \frac{s-5}{2(s-1)} .
$$

We now show that (8.7) cannot occur for $3 \leqslant s \leqslant 8$ : indeed, for any $1<s<9$, we have $(s-5) /(2(s-1))=\frac{1}{2}-2 /(s-1)<\frac{1}{4}$, therefore $a_{1}<\frac{1}{4}$. But then (8.6) says that

$$
0 \leqslant a_{2} \leqslant \frac{1}{2}\left(a_{1}^{2}-\frac{\left(1-a_{1}\right)^{2}}{s}\right)=\frac{a_{1}^{2}(s-1)+2 a_{1}-1}{2 s},
$$

so $a_{1}^{2}(s-1)+2 a_{1}-1 \geqslant 0$, implying either $a_{1} \leqslant 1 /(1-\sqrt{s})<0$ or $a_{1} \geqslant 1 /(1+\sqrt{s})>$ $1 /(1+\sqrt{9})=\frac{1}{4}$. The first case is ruled out by $a_{1} \geqslant 0$, and the second one is by $a_{1}<\frac{1}{4}$.

Hence the only possibility is (8.8) above. But then $\frac{1}{2} \leqslant a_{1} \leqslant\left(\begin{array}{l}s \\ 1\end{array}\right) / r^{1}=s / 2 s=\frac{1}{2}$ by Lemma 8.5, and so, again by the same lemma, we have $P(z)=(1+z / 2 s)^{s}$.

REMARK 8.8. Notice that [10] contains many uniqueness results for polynomials with maximal radius of absolute monotonicity, but those polynomials should approximate the exponential function near the origin to at least first order, that is, $P(0)=P^{\prime}(0)=1$ should hold. In Lemma 8.5, only $P(0)=1$ is required, whereas in Lemma 8.7 we assume $P(0)=1$ together with the non-linear condition (8.6). 


\subsection{Various remarks and questions}

REMARK 8.9. Let us fix $s \geqslant 3$. Regarding the structure of $\widehat{\Pi}_{s / s, p}$, we make the following observations. The non-trivial $p$ values are $2 \leqslant p \leqslant s+1$, but the $p=s+1$ case is special, because then $\widehat{\Pi}_{s / s, p}$ contains only finitely many functions. For $2 \leqslant p \leqslant s$, the general form of $\psi \in \widehat{\Pi}_{s / s, p}$ reads as

$$
\psi(z)=\frac{\sum_{m=0}^{p}\left(\sum_{k=0}^{m} \frac{(-1)^{k} a^{k}}{(m-k) !}\left(\begin{array}{l}
s \\
k
\end{array}\right)\right) z^{m}+\sum_{m=p+1}^{s} a_{m} z^{m}}{(1-a z)^{s}},
$$

with suitable real parameters $a, a_{p+1}, \ldots, a_{s}$, and, of course, with the usual convention that $\sum_{m=n}^{N}(\cdot)=0$ for $n>N$. Formula (2.5) is recovered if we choose $p=2$ in (8.9).

REMARK 8.10. The conjectured optimal $\psi$ (that is, the one with maximal radius of absolute monotonicity) in the $\widehat{\Pi}_{s / s, 2}$ class satisfies (8.9) with $a=1 / 2 s$, whereas the choice $a=\frac{1}{2}(1-$ $\sqrt{(s-1) /(s+1)})$ yields the optimum in the $\widehat{\Pi}_{s / s, 3}$ class. In view of $\S 2.3$, these values have been verified for $3 \leqslant s \leqslant 4$. See [3, formulae (3.1) and (3.2)] also.

Remark 8.11. The optimal $\psi$ rational function in the $\widehat{\Pi}_{s / s, 2}$ class for $1 \leqslant s \leqslant 4$ satisfies the relation $\psi(-z)=1 / \psi(z)$.

REMARK 8.12. In [5, Theorem 4.4], a necessary and sufficient condition is formulated for a general rational function $\psi$ satisfying assumptions (1)-(3) (see our $\S 1.1$ ) to be absolutely monotonic on an interval $[x, 0] \subset(-B(\psi), 0](x<0)$. This condition, among others, requires checking the positivity of the 'dominant coefficient' $c\left(\alpha_{0}, \mu\left(\alpha_{0}\right)\right)$, being the numerator in the partial fraction decomposition of $\psi$ corresponding to the positive real pole $\alpha_{0}$ in Definition 1.10 in our work, and having the highest pole order. It can be seen that $c\left(\alpha_{0}, \mu\left(\alpha_{0}\right)\right)>0$ is equivalent to our necessary condition (2.6) formulated in the $\widehat{\Pi}_{s / s, 2}$ class, if ' $\geqslant 0$ ' is replaced by ' $>0$ ' in (2.6). Moreover, ' $=0$ ' in (2.6) holds precisely if the numerator and denominator of $\psi$ have a common root. We add that we have not used [5, Theorem 4.4] directly, because the explicit construction of its functions $F(k, x), L(x)$ and $K(x)$ (cf. our Remark 1.15 also), now possibly depending on an additional parameter, would not be straightforward. Instead, we reproduce the 'partial fraction decomposition + factoring out the dominant term + checking the sign of the remainder in finitely many cases' idea behind the proof of [5, Theorem 4.4] only after the optimal parameter value $a^{*}$ and the optimal radius of absolute monotonicity $R_{s / s, p}$ (or $\widehat{R}_{s / s, p}$ ) have been conjectured.

REMARK 8.13 (On the explicit computation of $\psi^{(k)}$ ). The sum in (2.7) has a bounded number of terms for all $k \in \mathbb{N}$, yielding a nice representation of the derivatives of functions in $\widehat{\Pi}_{s / s, 2}$. For $\psi \in \Pi_{s / s, p}$, a similar representation would require a sum with unbounded number of terms as $k$ increases, which precludes the extension of our analysis for $\psi \in \widehat{\Pi}_{s / s, 2}$ to the more general class. Fortunately, the optimal $\psi \in \Pi_{2 / 2,3}$ is also an element of $\widehat{\Pi}_{2 / 2,3}$.

REMARK 8.14 (On the Mathematica implementations). This work could not have been completed without Mathematica, and especially, without one of its key commands in polynomial algebra, Reduce. Both in the conjecture and proof phase, Mathematica's abilities to manipulate high-order root objects symbolically, numerically or graphically played a crucial role. Throughout our investigations, we tried to apply symbolic methods to the fullest extent possible. At the same time, each pivotal formula was tested numerically with several parameter 
values (some by using different Mathematica algorithms). For typical checks we have used 25 digits of precision, but when huge coefficients were involved, we switched to 100 or 1000 digits of precision. Results of the symbolic computations and those given by the numerical algorithms were also compared graphically, and found to be in perfect agreement. In fact, as a by-product of our investigations we have discovered and reported a few minor Mathematica bugs, occurring with very low probability, in contexts when certain algebraic relations between different parameter values are satisfied.

In addition, most of the key computations performed by Reduce were later reproduced and checked 'manually'. Let us give here only one example. In $\S 4.2$, Reduce was able to solve the equation $B^{\prime}(a)=0$ within $2 \mathrm{~s}$. When we solved the same equation by elementary techniques without Reduce, the corresponding Mathematica notebook with all the details written out fully and saved as a PDF file occupied 280 pages. The final integer polynomial with both root objects $\varrho_{351}(a)$ and $\varrho_{353}(a)$ eliminated had degree 162 and leading coefficient $\approx 4 \cdot 10^{311}$. In view of the above, it is very surprising that the algebraic number $a_{35}^{*}$ is so simple: the reason is that the final polynomial of degree 162 admitted a nice factorization.

Let us close this section by posing two natural questions.

QUESTION. What properties, for example, what geometrical configuration of the roots and poles, of the rational function $\psi$ determine whether $R(\psi)=B(\psi)$ or $R(\psi)<B(\psi)$ ? If $R(\psi)<$ $B(\psi)$, then which roots of which derivatives of $\psi$ will limit the value of $R(\psi)$ in the sense of Theorem 1.13?

QUESTION (cf. the uniqueness of the optimal polynomials in [10], and our $\S 2$ ). Let us consider a given $(s, p)$ pair $(s \geqslant 1, p \geqslant 2)$. Is there always a unique rational function $\psi=P / Q \in \Pi_{s / s, p}$ (or $\psi=P / Q \in \widehat{\Pi}_{s / s, p}$ ) with $P(0)=Q(0)=1$ such that $R(\psi)=R_{s / s, p}\left(\right.$ or $R(\psi)=\widehat{R}_{s / s, p}$ )?

\section{Appendix}

In this section we list some auxiliary algebraic numbers, polynomials or functions that have appeared in certain more involved proofs.

A.1. Algebraic expressions in the proof of $R_{4 / 4,7}$

$$
\begin{aligned}
\varrho_{471}= & \operatorname{root}_{1}(252105,936390,1441629,1175608,534576,128352,12704) \approx-0.843194, \\
\varrho_{472}= & \operatorname{root}_{2}(252105,936390,1441629,1175608,534576,128352,12704) \approx-0.471357, \\
\varrho_{473}= & \operatorname{rot}_{2}(1,-30,390,-2760,11160,-25200,25200) \approx 7.64527, \\
\varrho_{474}= & \operatorname{rot}_{1}(1,-15,90,-210) \approx 5.64849, \\
P_{471}(a)= & 4593387934777490821322994 a^{10}-23474176816503998442760098 a^{9} \\
& +54351276637181597088697031 a^{8}-75708182541462463946985360 a^{7} \\
& +71791544313152743211806464 a^{6}-51339236908163135191695540 a^{5} \\
& +32383499796235996766706978 a^{4}-22447171953065668650772896 a^{3} \\
& -31555722050640962925153690 a^{2}-13278623731050880370830074 a \\
& -1667865061502294482628289, \\
\varrho_{475}= & \operatorname{rot}_{1}(2914539265575,14876524399779,33497711997246,43683499824678, \\
& 36368607954483,20052618149655,7324770907832,1709817444048, \\
& 231520801344,13859993824) \approx-0.850052,
\end{aligned}
$$




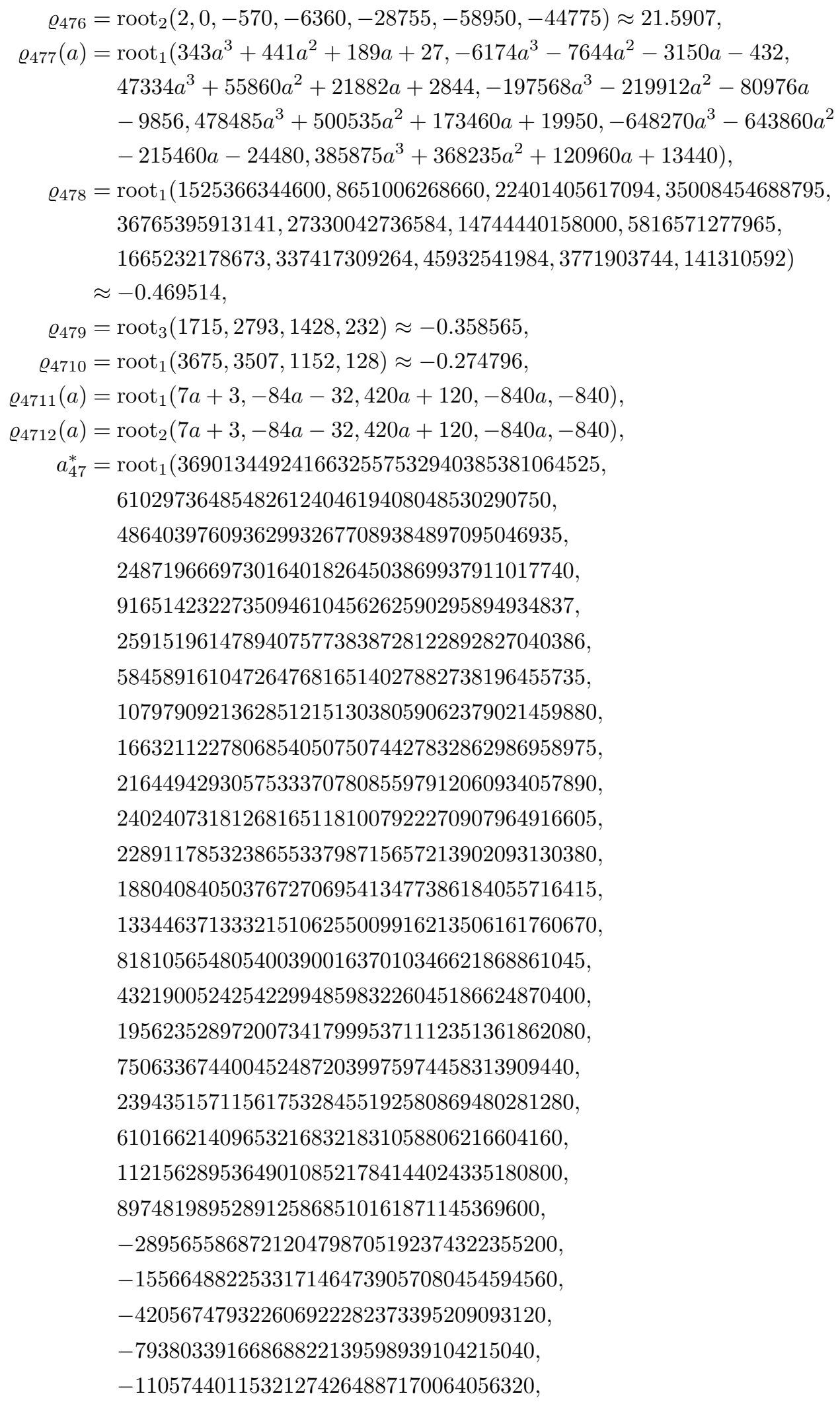




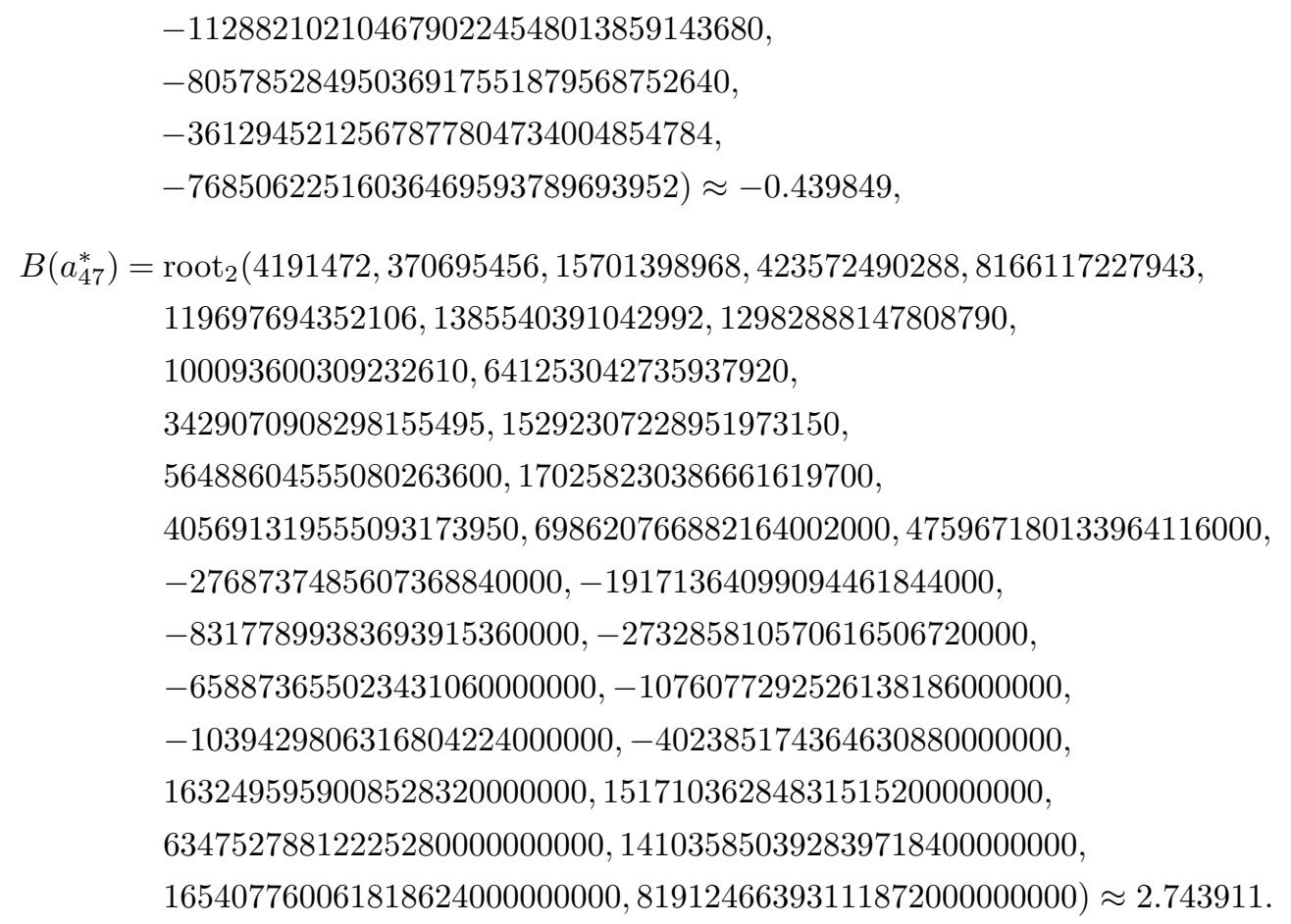

A.2. Algebraic expressions in the proof of $\widehat{R}_{4 / 4,2}$

$$
\begin{aligned}
P_{421}(a, c)= & 1536 a^{5}-1280 a^{4}-512 a^{3} c+436 a^{3}+576 a^{2} c-54 a^{2}-72 a c+2 a+c, \\
P_{422}(a, c)= & 2304 a^{4}-1728 a^{3}-1024 a^{2} c+480 a^{2}+768 a c-48 a-48 c+1, \\
P_{423}(a, c)= & 768 a^{3}-512 a^{2}-512 a c+104 a+192 c-7, \\
P_{424}(a, c)= & -384 a^{2}+224 a+512 c-25 \\
P_{425}(a)= & 512 a^{3}-7296 a^{2}+22800 a-17575, \\
\varrho_{421}= & \operatorname{root}_{1}(512,-7296,22800,-17575) \approx 1.17854, \\
P_{426}(a, c)= & 98304 a^{5}-679936 a^{4}+1266432 a^{3}+24576 a^{2} c-827264 a^{2} \\
& -153600 a c+156400 a+177600 c-6475, \\
P_{427}(a, c)= & 768 a^{5}-8064 a^{4}+20072 a^{3}+192 a^{2} c-15853 a^{2}-1824 a c+2888 a+2850 c, \\
P_{428}(a)= & 1024 a^{3}-10176 a^{2}+22048 a-11713 \\
\varrho_{422}= & \operatorname{root}_{1}(1024,-10176,22048,-11713) \approx 0.808208, \\
\varrho_{423}= & \operatorname{root}_{2}(1024,-10176,22048,-11713) \approx 1.97947, \\
P_{429}(a, c)= & 49152 a^{5}-245760 a^{4}+365312 a^{3}+12288 a^{2} c-210496 a^{2} \\
& -53248 a c+38272 a+42432 c-1547, \\
P_{4210}(a, c)= & 5406720 a^{5}-28110848 a^{4}-131072 a^{3} c+44025856 a^{3}+2605056 a^{2} c \\
& -26966400 a^{2}-8466432 a c+5517512 a+5997056 c-292825, \\
\varrho_{424}= & \operatorname{root}_{1}(256,-5088,16536,-11713) \approx 1.00126 .
\end{aligned}
$$

Acknowledgements. We are indebted to the referees of the manuscript for their suggestions and comments that helped us improving the presentation and arrangement of the material. 


\section{References}

1. C. Bolley and M. Crouzeix, 'Conservation de la positivité lors de la discrétisation des problémes d'évolution paraboliques', RAIRO Anal. Numér. 12 (1978) 237-245.

2. J. C. Butcher, Numerical methods for ordinary differential equations, 2nd edn (Wiley, 2008).

3. L. Ferracina and M. N. SpiJker, 'Strong stability of singly-diagonally-implicit Runge-Kutta methods', Appl. Numer. Math. 58 (2008) 1675-1686.

4. S. Gottlieb, D. I. Ketcheson and C.-W. Shu, Strong stability preserving Runge-Kutta and multistep time discretizations (World Scientific, 2011).

5. J. A. van de Griend and J. F. B. M. Kraaijevanger, 'Absolute monotonicity of rational functions occurring in the numerical solution of initial value problems', Numer. Math. 49 (1986) 413-424.

6. E. HAIRER and G. WANner, Solving ordinary differential equations II: stiff and differential-algebraic problems (Springer, 1991).

7. D. I. KetCheson, 'Computation of optimal monotonicity preserving general linear methods', Math. Comp. 78 (2009) 1497-1513.

8. D. I. KetCheson, 'High order strong stability preserving time integrators and numerical wave propagation methods for hyperbolic PDEs', PhD Thesis, University of Washington, 2009.

9. D. I. Ketcheson, C. B. Macdonald and S. Gottlieb, 'Optimal implicit strong stability preserving Runge-Kutta methods', Appl. Numer. Math. 59 (2009) 373-392.

10. J. F. B. M. KraAijevanger, 'Absolute monotonicity of polynomials occurring in the numerical solution of initial value problems', Numer. Math. 48 (1986) 303-322.

11. J. F. B. M. KraAijevanger, 'Contractivity of Runge-Kutta Methods', BIT 31 (1991) 482-528.

12. M. N. SpIJKER, 'Contractivity in the numerical solution of initial value problems', Numer. Math. 42 (1983) 271-290.

Lajos Lóczi

Computer, Electrical and

Mathematical Sciences and Engineering

King Abdullah University of

Science and Technology (KAUST)

4700 KAUST, Thuwal, 23955

Saudi Arabia

lajos.loczi@kaust.edu.sa
David I. Ketcheson

Computer, Electrical and

Mathematical Sciences and Engineering

King Abdullah University of

Science and Technology (KAUST)

4700 KAUST, Thuwal, 23955

Saudi Arabia

david.ketcheson@kaust.edu.sa 